TRANSACTIONS OF THE

AMERICAN MATHEMATICAL SOCIETY

Volume 362, Number 8, August 2010, Pages 3997-4043

S 0002-9947(10)05073-7

Article electronically published on March 23, 2010

\title{
MORSE-BOTT HOMOLOGY
}

\author{
AUGUSTIN BANYAGA AND DAVID E. HURTUBISE
}

\begin{abstract}
We give a new proof of the Morse Homology Theorem by constructing a chain complex associated to a Morse-Bott-Smale function that reduces to the Morse-Smale-Witten chain complex when the function is MorseSmale and to the chain complex of smooth singular $N$-cube chains when the function is constant. We show that the homology of the chain complex is independent of the Morse-Bott-Smale function by using compactified moduli spaces of time dependent gradient flow lines to prove a Floer-type continuation theorem.
\end{abstract}

\section{INTRODUCTION}

1.1. Overview. Let $C r(f)=\left\{p \in M \mid d f_{p}=0\right\}$ denote the set of critical points of a smooth function $f: M \rightarrow \mathbb{R}$ on a smooth $m$-dimensional manifold $M$. A critical point $p \in C r(f)$ is said to be non-degenerate if and only if $d f$ is transverse to the zero section of $T^{*} M$ at $p$. In local coordinates this is equivalent to the condition that the $m \times m$ Hessian matrix $\left(\frac{\partial^{2} f}{\partial x_{i} \partial x_{j}}\right)$ has rank $m$ at $p$. If all the critical points of $f$ are non-degenerate, then $f$ is called a Morse function.

A Morse function $f: M \rightarrow \mathbb{R}$ on a finite-dimensional compact smooth Riemannian manifold $(M, g)$ is called Morse-Smale if all its stable and unstable manifolds intersect transversally. Such a function gives rise to a chain complex $\left(C_{*}(f), \partial_{*}\right)$, called the Morse-Smale-Witten chain complex. The chains of this complex are generated by the critical points of $f$, and the boundary operator is defined by counting gradient flow lines between critical points (with sign). The Morse Homology Theorem says that the homology of the Morse-Smale-Witten chain complex is isomorphic to the singular homology of $M$ with integer coefficients [7, [41.

If $C r(f)$ is a disjoint union of finitely many connected submanifolds and the Hessian of $f$ is non-degenerate in the direction normal to $\operatorname{Cr}(f)$, then $f$ is called a Morse-Bott function [11. In this paper we construct a new chain complex associated

Received by the editors October 11, 2007.

2010 Mathematics Subject Classification. Primary 57R70; Secondary 58E05, 57R58, 37D15.

(C)2010 American Mathematical Society Reverts to public domain 28 years from publication 
to a Morse-Bott function meeting certain transversality requirements (see Definition 3.4) that generalizes the Morse-Smale-Witten chain complex. This singular MorseBott-Smale chain complex has the following form:

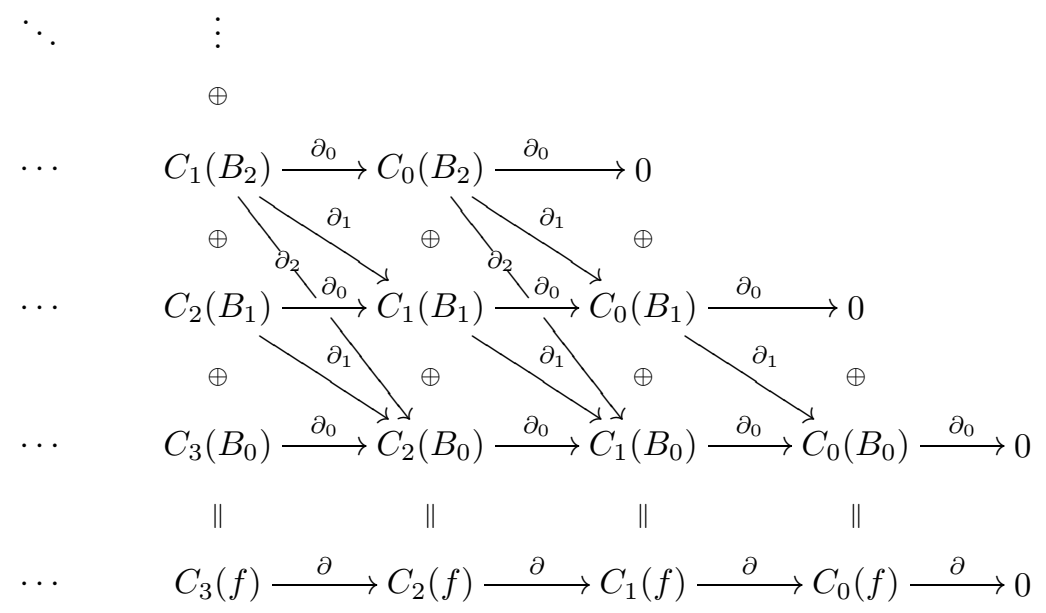

where $C_{p}\left(B_{i}\right)$ is the group of " $p$-dimensional chains" in the critical submanifolds of Morse-Bott index $i$, and the boundary operator is defined as a sum of homomorphisms $\partial=\partial_{0} \oplus \cdots \oplus \partial_{m}$.

When the function $f: M \rightarrow \mathbb{R}$ is Morse-Smale the critical set $B_{i}$ is a discrete set of points for all $i=0, \ldots, m$, and the groups $C_{p}\left(B_{i}\right)$ are trivial for all $p>0$. When the function is constant the entire manifold $M$ is a critical submanifold of Morse-Bott index zero. In this case $B_{i}=\emptyset$ for all $i>0$, and the groups $C_{p}\left(B_{i}\right)$ are trivial for all $i>0$. These two cases appear in the above diagram of a general Morse-Bott-Smale chain complex as follows:

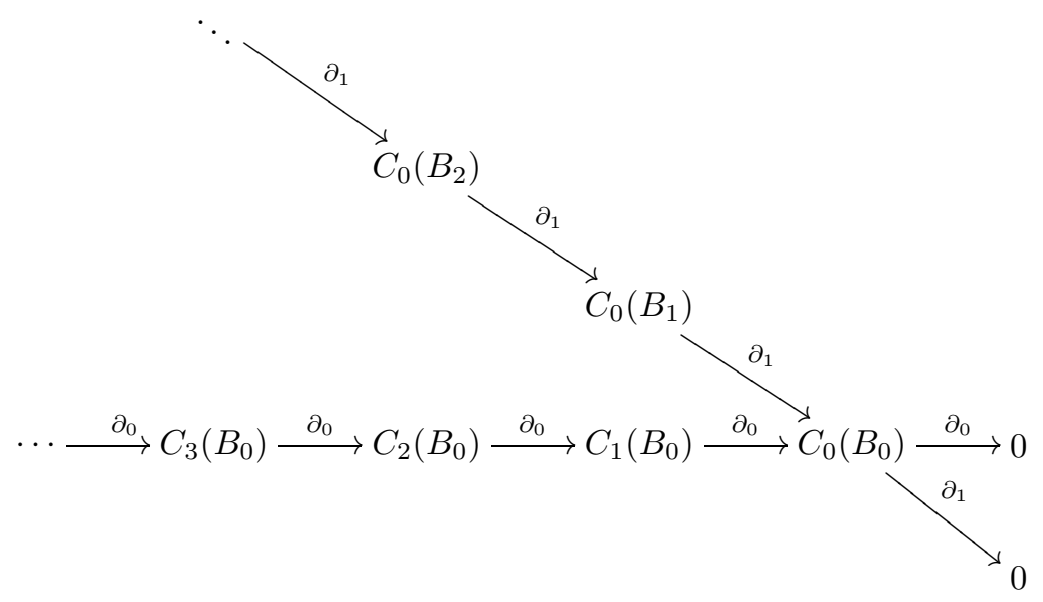

In the first case the homomorphism $\partial_{1}$ is the Morse-Smale-Witten boundary operator, and in the second case the homomorphism $\partial_{0}$ is comparable to the boundary operator on singular cubical chains found in 35. Thus, the Morse-Bott-Smale chain complex provides a means of interpolating between the Morse-Smale-Witten 
chain complex and the chain complex of singular cubical chains. Moreover, a Floertype continuation theorem shows that the homology of the Morse-Bott-Smale chain complex is independent of the Morse-Bott-Smale function $f: M \rightarrow \mathbb{R}$ and gives a new proof of the Morse Homology Theorem.

Any Morse function can be smoothly deformed to a constant function (which is a Morse-Bott function), and any Morse-Bott function $f: M \rightarrow \mathbb{R}$ can be smoothly perturbed to a Morse function since the space of smooth Morse functions on $M$ is an open dense subspace of the space $C^{\infty}(M, \mathbb{R})$ of all smooth functions on $M$. In fact, one can define an explicit perturbation of a Morse-Bott function $f: M \rightarrow \mathbb{R}$ to a Morse function using Morse functions defined on the critical submanifolds [4]. These sorts of explicit perturbations can be quite useful for simplifying computations, since the boundary operator of the chain complex associated to the perturbed function is given by $\partial=\partial_{1}$ with all the other homomorphisms $\partial_{j}=0$ for $j \neq 1$. An example of this approach can be found in [6], where the Morse-Bott inequalities are proved without reference to the full Morse-Bott-Smale chain complex discussed in this paper.

Although perturbing a Morse-Bott function to a Morse function greatly simplifies the Morse-Bott-Smale chain complex, i.e. $\partial_{j}=0$ for $i \neq 1$, there are cases where it is undesirable to perturb the Morse-Bott function. For instance, there are certain Morse-Bott functions that arise naturally in contexts where there is some sort of symmetry or a group action where the perturbation would destroy the symmetry or be incompatible with the group action [1, 22, 3], [5, 12, [16, [17, 23], 31. In these cases it is important to have the full Morse-Bott-Smale chain complex defined by the given Morse-Bott function, even though $\partial_{j}$ may not be zero for $j \neq 1$.

Heuristically, the homomorphisms $\partial_{j}: C_{p}\left(B_{i}\right) \rightarrow C_{p+j-1}\left(B_{i-j}\right)$ for $j=1, \ldots, m$ can be described as follows. Imagine that every topological space under consideration has a preferred triangulation. Then a map $\sigma: P \rightarrow B_{i}$ from some triangulated space $P$ of dimension $p$ would determine a chain in the singular chain group $S_{p}\left(B_{i} ; \mathbb{Z}\right)$. Using $\sigma: P \rightarrow B_{i}$ we could form a fibered product with respect to the beginning point map $\partial_{-}: \overline{\mathcal{M}}\left(B_{i}, B_{i-j}\right) \rightarrow B_{i}$ from the compactified moduli space of gradient flow lines from $B_{i}$ to $B_{i-j}$, and then by projecting onto the second component of the fibered product and composing with the endpoint map $\partial_{+}: \overline{\mathcal{M}}\left(B_{i}, B_{i-j}\right) \rightarrow B_{i-j}$ we would have a map

$$
\partial_{j}: P \times_{B_{i}} \overline{\mathcal{M}}\left(B_{i}, B_{i-j}\right) \stackrel{\pi_{2}}{\longrightarrow} \overline{\mathcal{M}}\left(B_{i}, B_{i-j}\right) \stackrel{\partial_{+}}{\longrightarrow} B_{i-j} .
$$

The dimension of $\overline{\mathcal{M}}\left(B_{i}, B_{i-j}\right)$ is $b_{i}+j-1$, where $b_{i}=\operatorname{dim} B_{i}$, and (assuming some transversality conditions) the dimension of the fibered product $P \times_{B_{i}} \overline{\mathcal{M}}\left(B_{i}, B_{i-j}\right)$ is $p+j-1$. Thus, if we were to assume that the fibered product also has a preferred triangulation, then the above map would determine a singular chain in the singular chain group $S_{p+j-1}\left(B_{i-j} ; \mathbb{Z}\right)$.

Of course, a topological space does not necessarily have a preferred triangulation. One approach to making the above heuristic discussion rigorous would be to show that all the spaces under consideration can be triangulated in some way and then prove that the homology of the resulting complex does not depend on the chosen triangulations. However, this approach would involve picking triangulations on an uncountably infinite number of spaces, namely all the fibered products $P \times_{B_{i}}$ $\overline{\mathcal{M}}\left(B_{i}, B_{i-j}\right)$. This approach seems quite daunting in light of the fact that there are 
simple diagrams of polyhedra and piecewise linear maps that are not triangulable:

$$
Y \stackrel{\sigma_{1}}{\longleftarrow} X \stackrel{\sigma_{2}}{\longrightarrow} Z
$$

That is, there may not exist triangulations of $X, Y$, and $Z$ with respect to which both $\sigma_{1}$ and $\sigma_{2}$ are simplicial [15]. (See also Example 5.17] in Subsection [5.5 of this paper.)

In light of these observations, our approach to constructing a singular MorseBott-Smale chain complex is not based on picking triangulations, but on defining singular chain complexes where the generators are allowed to have a variety of domains. We generalize the usual singular chain complexes based on maps from the standard $p$-simplex $\Delta^{p} \subset \mathbb{R}^{p+1}$ (or the unit $p$-cube $I^{p}$ ) to allow maps from the faces of an $N$-cube (for some large $N$ ), the endpoint maps from compactified moduli spaces of gradient flow lines, and the endpoint maps from their fibered products. We call these generalizations of $\Delta^{p}$ abstract topological chains, and we refer to the corresponding singular chains as singular topological chains. Thus, the maps

$$
\partial_{j}: P \times_{B_{i}} \overline{\mathcal{M}}\left(B_{i}, B_{i-j}\right) \stackrel{\pi_{2}}{\longrightarrow} \overline{\mathcal{M}}\left(B_{i}, B_{i-j}\right) \stackrel{\partial_{+}}{\longrightarrow} B_{i-j}
$$

for $j=1, \ldots, m$ described above are singular topological chains in our new framework (without any need for a triangulation on the domain). However, the homomorphism $\partial_{0}$ is no longer the "usual" boundary operator on singular chains, since the generators have a variety of domains.

Of course, a singular chain complex with maps defined on a variety of domains will contain more maps than a singular chain complex where all the $p$-chains are defined on the domain $\Delta^{p}$, and some redundancy may appear in the larger chain complex. Hence, we define degeneracy relations to identify singular chains that are "essentially" the same but defined on different domains. For example, fix a large integer $N$ and let $C_{p}$ be the $p$-dimensional faces of the unit $N$-cube $I^{N}$. There are a large number of elements in $C_{p}$ (which are all "essentially" the same), and different elements may support singular chains that are "essentially" the same. For instance, any element $P \in C_{p}$ is homeomorphic to the standard unit $p$-cube $I^{p}$ via a homeomorphism $\alpha: P \rightarrow I^{p}$, and the usual singular boundary operator on a singular $p$-cube $\sigma: I^{p} \rightarrow B$ in a topological space $B$ induces a singular boundary operator on the singular $C_{p}$-space $\sigma \circ \alpha: P \rightarrow B$ such that the boundary operators commute with the homeomorphism $\alpha: P \rightarrow I^{p}$. So, $\sigma: I^{p} \rightarrow B$ and $\sigma \circ \alpha: P \rightarrow B$ are "essentially" the same, even though they are defined on different domains.

More precisely, to construct our singular Morse-Bott-Smale chain complex we begin by defining the set $C_{p}$ of allowed domains of degree $p$ for all $p$, and then we define a boundary operator on the free abelian group generated by the elements of $C_{p}$, i.e. $S_{p}=\mathbb{Z}\left[C_{p}\right]$. This boundary operator

$$
\partial: S_{p} \rightarrow S_{p-1}
$$

induces a boundary operator

$$
\partial: S_{p}(B) \rightarrow S_{p-1}(B)
$$

on singular $C_{p^{-}}$-chains in $B$ as follows. If $\sigma: P \rightarrow B$ is a singular $C_{p}$-space in $B$, then $\partial(\sigma)$ is given by the formula

$$
\partial(\sigma)=\left.\sum_{k} n_{k} \sigma\right|_{P_{k}}
$$


where

$$
\partial(P)=\sum_{k} n_{k} P_{k}
$$

and $n_{k}= \pm 1$ for all $k$. The degeneracy relations define a subgroup $D_{p}(B) \subseteq S_{p}(B)$ of degenerate singular topological chains in $B$ such that $\partial\left(D_{p}(B)\right) \subseteq D_{p-1}(B)$, and hence the boundary operator on singular $C_{p}$-chains in $B$ descends to a boundary operator on the quotient groups:

$$
\partial: S_{p}(B) / D_{p}(B) \rightarrow S_{p-1}(B) / D_{p-1}(B) .
$$

This describes our framework for defining the homomorphism $\partial_{0}$ in the MorseBott-Smale chain complex. In fact, in the above diagram for a general Morse-BottSmale chain complex we have

$$
C_{p}\left(B_{i}\right)=S_{p}^{\infty}\left(B_{i}\right) / D_{p}^{\infty}\left(B_{i}\right),
$$

where $S_{p}^{\infty}\left(B_{i}\right) \subset S_{p}\left(B_{i}\right), D_{p}^{\infty}\left(B_{i}\right) \subset D_{p}\left(B_{i}\right)$, and $\partial_{0}: C_{p}\left(B_{i}\right) \rightarrow C_{p-1}\left(B_{i}\right)$ is $(-1)^{p+i}$ times the boundary operator on singular topological chains developed in Section 4 of this paper (see Definitions 5.2, 5.4, and 5.11). The homomorphisms $\partial_{1}, \ldots, \partial_{m}$ are then defined as above using fibered products of compactified moduli spaces, and it is shown that they preserve the groups of degenerate singular topological chains (see Lemma 5.10). Hence there are induced homomorphisms

$$
\partial_{j}: S_{p}^{\infty}\left(B_{i}\right) / D_{p}^{\infty}\left(B_{i}\right) \rightarrow S_{p+j-1}^{\infty}\left(B_{i-j}\right) / D_{p+j-1}^{\infty}\left(B_{i-j}\right)
$$

for all $j=0, \ldots, m$, and these induced homomorphisms fit together to form the boundary operator $\partial=\partial_{0} \oplus \cdots \oplus \partial_{m}$ in the Morse-Bott-Smale chain complex.

1.2. Comparison with other approaches. The Morse-Bott-Smale chain complex constructed in this paper is inspired by several other authors (44, 22, 23, [32, 33, and [39]) who have studied both the finite-dimensional case addressed here and the infinite-dimensional version of Floer-Bott homology.

However, the approach taken in this paper is fundamentally different and, in our opinion, more straightforward and more like singular homology than previous approaches. For instance, other authors use the machinery of spectral sequences; cf. [4], 23], and [39]. In contrast, the approach taken here does not require spectral sequences (although several of the diagrams found in Section 5 are inspired by and resemble first quadrant spectral sequences).

Moreover, as a novel feature, the Morse-Bott-Smale chain complex defined in Section 5 provides a common framework for encoding both smooth singular cubical chains and Morse-Smale-Witten chains. In particular, it reduces to the chain complex of smooth singular $N$-cube chains when the function is constant (Example [5.13), and it reduces to the Morse-Smale-Witten chain complex when the function is Morse-Smale (Example 5.14). This is quite different from the approaches that count flow lines with cascades [22], where the chain complex reduces to the Morse-Smale-Witten chain complex for both Morse-Smale functions and constant functions.

To construct a Morse-Bott-Smale chain (or cochain) complex, one must first choose a category for the objects in the chain (or cochain) complex. For instance, Austin and Braam [4 use differential forms on the critical submanifolds to define the groups $C_{p}\left(B_{i}\right)$ in their Morse-Bott-Smale cochain complex, and they define the homomorphisms $\partial_{1}, \ldots, \partial_{m}$ in their cochain complex using integration along 
the fibers. They then use the machinery of spectral sequences to prove that the cochain complex they construct computes the de Rahm cohomology of the manifold.

In contrast, Latschev [32] views the stable and unstable manifolds (and their fibered products) as currents. Latschev shows (following [25]) that these currents determine an operator (from differential forms to currents) that is chain homotopic to the inclusion. After showing that this operator can be extended to singular chains that are "sufficiently transverse" to the unstable manifolds, Latschev shows that the image of the extended operator is the set of "stable bundles of smooth chains" in the critical submanifolds. A formula for the differential on the chain complex whose groups consist of stable bundles over chains in the critical submanifolds is given, and it is proved that the homology of this (Morse-Bott-Smale) chain complex is isomorphic to the singular homology of the manifold with integer coefficients.

Another approach to Morse-Bott homology can be found in a paper by Fukaya [23], where the Floer-Bott homology of the Chern-Simons functional is studied. The groups in Fukaya's version of Morse-Bott homology are generated by piecewise smooth maps from objects he calls "abstract geometric chains" and attributes to Gromov 24]. Similarly, Ruan and Tian's version of "Bott-type symplectic Floer cohomology" 39] is based on these same "abstract geometric chains", which they attribute to Fukaya 23. According to Fukaya, an "abstract geometric chain" is a finite simplicial complex (see Definition 1.14 of 23 ) that satisfies certain conditions, including a codimension 2 boundary condition, reminiscent of the conditions used to define "pseudocycles" (see [30, [36], and [42]). The boundary operator in the chain complex described by Fukaya is defined on the set of "transversal geometric chains" by taking fibered products with moduli spaces of gradient flow lines, and Theorem 1.2 of [23] says that there is a spectral sequence associated to a "generic" Morse-Bott function which converges to the singular homology of the manifold.

In this paper, we construct our Morse-Bott-Smale chain complex over the category of compact oriented smooth manifolds with corners. This is a natural choice of category for a Morse-Bott-Smale chain complex because the compactified moduli spaces of gradient flow lines of a Morse-Bott-Smale function are compact oriented smooth manifolds with corners (see Theorem 4.9). Moreover, we prove in Subsection 5.5 that all the relevant fibered products are compact smooth manifolds with corners. Hence, the fibered product constructions used in this paper stay within the chosen category for the objects in the chain complex.

1.3. Outline of the paper. In Section 2 we recall some basic facts about the Morse-Smale-Witten chain complex, and in Section 3 we recall some basic facts about Morse-Bott functions and state the Morse-Bott-Smale transversality condition.

Section 4 establishes the framework we use to define the homomorphism $\partial_{0}$ in the Morse-Bott-Smale chain complex constructed in Section 5.

In Subsection 4.1 we introduce the notion of abstract topological chains and singular topological chains, which generalize the usual singular chain complexes based on maps from the standard $p$-simplex $\Delta^{p} \subset \mathbb{R}^{p+1}$ or the unit $p$-cube $I^{p}$. Abstract topological chains are introduced in order to provide the flexibility of constructing singular chain complexes with maps from a variety of domains. To construct the Morse-Bott-Smale chain complex in Section 5, we need to allow maps from the faces of an $N$-cube (for some large $N$ ), the compactified moduli spaces of gradient flow lines, and their fibered products. 
The formalism of abstract topological chains and singular topological chains is very general and provides a natural framework for some of the formulas found in 23 .

In Subsection 4.2 we show how the faces of an $N$-cube can be viewed as abstract topological chains. Using the degeneracy relations in Definition 4.3, this produces the chain complex of singular $N$-cube chains, which is comparable to the singular cubical chain complex of [35] (see Theorem 4.4). The reader should note that in Subsection 4.2 the singular topological chain group $S_{p}(B)$ is generated by maps from the $p$-dimensional faces of the unit $N$-cube. So, the maps in $S_{p}(B)$ are defined on several different domains. However, these domains are identified via the subgroup $D_{p}(B)$ of degenerate singular topological chains. Hence, every equivalence class in $S_{p}(B) / D_{p}(B)$ contains a representative defined on the standard $p$-cube $I^{p} \times$ $(0, \ldots, 0) \subseteq I^{N}$.

In Subsection 4.3 we define the fibered product of singular topological chains and show that the fibered product of singular topological chains is an abstract topological chain. The formal constructions in Subsection 4.3 are used in Subsection 4.4, where the abstract topological chain structure on compactified moduli spaces of gradient flow lines is defined. This abstract topological chain structure is defined in terms of fibered products (see Definition 4.11), and the general results proved in Subsection 4.3 are used to prove that the degree and boundary operator for compactified moduli spaces of gradient flow lines satisfy the axioms for abstract topological chains (see Lemma 4.12).

In Section 5 we construct our Morse-Bott-Smale chain complex $\left(C_{*}(f), \partial\right)$. The construction begins by defining the set $C_{p}$ of allowed domains for the singular topological chains. The set of allowed domains $C_{p}$ includes the $p$-dimensional faces of the unit $N$-cube $I^{N}$ and the $p$-dimensional connected components of fibered products with compactified moduli spaces of gradient flow lines. Subsection [5.5] is devoted to the proof of Lemma 5.1. which says that the elements of $C_{p}$ are compact oriented smooth manifolds with corners.

In Subsection 5.1 we define the chain complex $\left(\tilde{C}_{*}(f), \partial\right)$. The groups in the Morse-Bott-Smale chain complex $\left(C_{*}(f), \partial\right)$ are defined in Definition 5.11 as the quotient of the groups in $\left(\tilde{C}_{*}(f), \partial\right)$ by the degenerate singular topological chains. These degenerate singular topological chains are defined in Subsection 5.3 using the orientation conventions given in Subsection 5.2. The degenerate singular topological chains defined in Subsection 5.3 include the degenerate singular $N$-cube chains from Subsection 4.2 together with additional chains having domains that are components of fibered products. The degenerate singular topological chains are used to identify chains that are "essentially" the same, but defined on different domains.

The chain complex $\left(\tilde{C}_{*}(f), \partial\right)$ defined in Subsection $[5.1$ has a natural filtration determined by the Morse-Bott index, and the boundary operator decomposes into a sum of differentials $\partial=\bigoplus_{j=0}^{m} \partial_{j}$, where $\partial_{j}$ is a homomorphism that decreases the Morse-Bott index by $j$. In fact, in Subsection 5.1 the homomorphisms $\partial_{j}$ are defined first, and then the full boundary operator is defined to be $\partial=\bigoplus_{j=0}^{m} \partial_{j}$. Thus, the chain complex $\left(\tilde{C}_{*}(f), \partial\right)$ looks like a first quadrant spectral sequence where the homomorphisms $\partial_{j}$ are all defined on the $E^{0}$ term (see Definition [5.11). More precisely, the chain complex $\left(\tilde{C}_{*}(f), \partial\right)$ is multicomplex [29], [37].

The homomorphism $\partial_{0}$ in Subsection 5.1 is defined in terms of the singular topological chain structure discussed in Section 4 , and the homomorphisms $\partial_{1}, \ldots, \partial_{m}$ 
are defined using fibered products of compactified moduli spaces of gradient flow lines. It is shown in Lemma 5.10 that these homomorphisms preserve the groups of degenerate singular topological chains, and hence they induce homomorphisms (still denoted by $\partial_{0}, \ldots, \partial_{m}$ ) on the groups in the Morse-Bott-Smale chain complex $\left(C_{*}(f), \partial\right)$. Thus, Corollary $\left[5.6\right.$ implies that $\partial^{2}=0$ for the Morse-Bott-Smale chain complex $\left(C_{*}(f), \partial\right)$. In Subsection 5.4 we give several examples of computing Morse-Bott homology, including the cases where the function is constant (Example 5.13) and where the function is Morse-Smale (Example 5.14).

Section [6 is devoted to proving a Floer-type continuation theorem (Theorem 6.17) that shows that the homology of the Morse-Bott-Smale chain complex defined in Section $[5$ is independent of the Morse-Bott-Smale function $f: M \rightarrow \mathbb{R}$ used to define the complex. The proof follows standard arguments involving compactified moduli spaces of time dependent gradient flow lines that can be found in [4, 9], [18, 19], 41], and 44]. (An outline of the proof of the continuation theorem can be found at the beginning of Section [6) When the Morse-Bott-Smale function $f: M \rightarrow \mathbb{R}$ is constant, the entire manifold $M$ is a critical submanifold of MorseBott index zero, and the Morse-Bott-Smale chain complex defined in Section 5 reduces to the chain complex of smooth singular $N$-cube chains (Example 5.13). Hence, Theorem 6.17 implies that, for any Morse-Bott-Smale function $f: M \rightarrow \mathbb{R}$, the homology of the Morse-Bott-Smale chain complex associated to $f$ is isomorphic to the singular homology of the manifold with integer coefficients. Moreover, when the function $f: M \rightarrow \mathbb{R}$ is Morse-Smale, the complex reduces to the Morse-SmaleWitten chain complex (Example 5.14). Thus, the Morse Homology Theorem follows as a corollary to the results in this paper.

Other authors have shown that the theory of Morse-Bott homology can be applied to a variety of problems in algebraic topology, Floer homology, gauge theory, and quantum cohomology. For instance, applications to equivariant homology/cohomology and cup products are discussed in 4] and 32], applications to the Floer homology of the Chern-Simons functional and Donaldson polynomials are discussed in 5] and 23, and applications to symplectic Floer homology and quantum cohomology are discussed in 33 and 39. Although we do not discuss applications of the Morse-Bott-Smale chain complex constructed in Sections 4 and 5. the techniques developed in this paper were designed with the above applications in mind. Thus, we expect that our approach to Morse-Bott homology can be applied to problems such as the ones listed above and can be readily extended to the infinite-dimensional case of Floer-Bott homology.

\section{The Morse-Smale-Witten chain complex}

In this section we briefly recall the construction of the Morse-Smale-Witten chain complex and the Morse Homology Theorem. For more details we refer the reader to 7 .

Let $f: M \rightarrow \mathbb{R}$ be a Morse function on a finite-dimensional compact smooth Riemannian manifold $(M, g)$. The stable manifold of a critical point $p \in C r(f)$ is defined to be

$$
W^{s}(p)=\left\{x \in M \mid \lim _{t \rightarrow \infty} \varphi_{t}(x)=p\right\},
$$

where $\varphi_{t}$ is the 1-parameter group of diffeomorphisms generated by minus the gradient vector field, i.e. $-\nabla f$. Similarly, the unstable manifold of $p$ is defined 
to be

$$
W^{u}(p)=\left\{x \in M \mid \lim _{t \rightarrow-\infty} \varphi_{t}(x)=p\right\} .
$$

The Stable/Unstable Manifold Theorem for a Morse function says that the tangent space at $p$ splits as

$$
T_{p} M=T_{p}^{s} M \oplus T_{p}^{u} M
$$

where the Hessian is positive definite on $T_{p}^{s} M$ and negative definite on $T_{p}^{u} M$. Moreover, the stable and unstable manifolds of $p$ are the surjective images of smooth embeddings

$$
\begin{gathered}
E^{s}: T_{p}^{s} M \rightarrow W^{s}(p) \subseteq M, \\
E^{u}: T_{p}^{u} M \rightarrow W^{u}(p) \subseteq M .
\end{gathered}
$$

Hence, $W^{s}(p)$ is a smoothly embedded open disk of dimension $m-\lambda_{p}$, and $W^{u}(p)$ is a smoothly embedded open disk of dimension $\lambda_{p}$ where $m$ is the dimension of $M$ and $\lambda_{p}$ is the index of the critical point $p$, i.e. the dimension of $T_{p}^{u} M$.

If the stable and unstable manifolds of a Morse function $f: M \rightarrow \mathbb{R}$ all intersect transversally, then the function is called Morse-Smale. The next result describes the abundance of Morse-Smale functions.

Theorem 2.1 (Kupka-Smale Theorem). If $(M, g)$ is a finite-dimensional compact smooth Riemannian manifold, then the space of all $C^{r}$ Morse-Smale functions on $M$ is a dense subspace of $C^{r}(M, \mathbb{R})$ for any $2 \leq r \leq \infty$.

If $f: M \rightarrow \mathbb{R}$ is a Morse-Smale function, then using Palis' $\lambda$-Lemma one can show that there is a partial ordering on the critical points of $f$ defined as follows.

Definition 2.2. Let $p$ and $q$ be critical points of $f: M \rightarrow \mathbb{R}$. We say that $q$ is succeeded by $p, q \succeq p$, if and only if $W(q, p)=W^{u}(q) \cap W^{s}(p) \neq \emptyset$, i.e. there exists a gradient flow line from $q$ to $p$.

Note that $W(q, p)$ is an embedded submanifold of $M$ of dimension $\lambda_{q}-\lambda_{p}$. Hence, $q \succeq p$ implies that $\lambda_{q} \geq \lambda_{p}$. As other consequences of Palis' $\lambda$-Lemma we have the following.

Theorem 2.3. If $p$ and $q$ are critical points of $f: M \rightarrow \mathbb{R}$ such that $q \succeq p$, then

$$
\overline{W(q, p)}=\overline{W^{u}(q)} \cap \overline{W^{s}(p)}=\bigcup_{q \succeq \tilde{q} \succeq \tilde{p} \succeq p} W(\tilde{q}, \tilde{p}),
$$

where the union is over all critical points between $q$ and $p$.

Corollary 2.4. If $p$ and $q$ are critical points of relative index one, i.e. if $\lambda_{q}-\lambda_{p}=1$ and $q \succeq p$, then

$$
\overline{W(q, p)}=W(q, p) \cup\{p, q\} .
$$

Moreover, $W(q, p)$ has finitely many components, i.e. the number of gradient flow lines from $q$ to $p$ is finite.

If we assume that $M$ is oriented and we choose an orientation for each of the unstable manifolds of $f$, then there is an induced orientation on the stable manifolds. The preceding corollary shows that we can then define an integer $n(q, p)$ associated to any two critical points $p$ and $q$ of relative index one by counting the number of gradient flow lines from $q$ to $p$ with signs determined by the orientations. The Morse-Smale-Witten chain complex is defined to be the chain complex 
$\left(C_{*}(f), \partial_{*}\right)$, where $C_{k}(f)$ is the free abelian group generated by the critical points $q$ of index $k$ and the boundary operator $\partial_{k}: C_{k}(f) \rightarrow C_{k-1}(f)$ is given by

$$
\partial_{k}(q)=\sum_{p \in C r_{k-1}(f)} n(q, p) p .
$$

Theorem 2.5 (Morse Homology Theorem). The pair $\left(C_{*}(f), \partial_{*}\right)$ is a chain complex, and its homology is isomorphic to the singular homology $H_{*}(M ; \mathbb{Z})$.

Note that the Morse Homology Theorem implies that the homology of $\left(C_{*}(f), \partial_{*}\right)$ is independent of the Morse-Smale function $f: M \rightarrow \mathbb{R}$, the Riemannian metric $g$, and the orientations.

\section{Morse-Bott Functions And transversality}

Let $f: M \rightarrow \mathbb{R}$ be a smooth function on a finite-dimensional compact smooth Riemannian manifold $(M, g)$. Assume that the critical point set

$$
C r(f)=\left\{p \in M \mid d f_{p}=0\right\}=\coprod_{\alpha=1}^{n} B_{\alpha}
$$

is a finite disjoint union of connected submanifolds $B_{\alpha}$ in $M$, called critical submanifolds. For any $p \in B$, a critical submanifold, the tangent space of $M$ splits as

$$
T_{p} M \approx T_{p} B \oplus \nu_{p}(B),
$$

where $\nu_{p}(B)$ denotes the normal bundle of $B$ in $M$. For $V, W \in T_{p} M$, the Hessian is a symmetric bilinear form defined by $\operatorname{Hess}_{p}(f)(V, W)=V_{p}(\tilde{W}(f))$ where $\tilde{W}$ is any extension of $W$ to a neighborhood of $p$. If $V \in T_{p} B$, then $H_{e s s}(f)(V, W)=0$ because $\tilde{W}_{x}(f)=0$ for all $x \in B$. Thus the Hessian determines a symmetric bilinear form on the normal space

$$
\operatorname{Hess}_{p}^{\nu}(f): \nu_{p}(B) \times \nu_{p}(B) \rightarrow \mathbb{R} .
$$

Definition 3.1. A function $f: M \rightarrow \mathbb{R}$ is said to be Morse-Bott if and only if the critical point set $C r(f)$ is a finite disjoint union of connected submanifolds of $M$ and for each connected submanifold $B \subseteq C r(f)$ the normal Hessian $\operatorname{Hess}_{p}^{\nu}(f)$ is non-degenerate for all $p \in B$. The index $\lambda_{p}$ of a critical point $p \in B \subseteq \operatorname{Cr}(f)$ is defined to be the maximal dimension of a subspace of $\nu_{p}(B)$ on which $\operatorname{Hess}_{p}^{\nu}(f)$ is negative definite.

For a proof of the following lemma see Section 3.5 of [7] or [8].

Lemma 3.2 (Morse-Bott Lemma). Let $f: M \rightarrow \mathbb{R}$ be a Morse-Bott function, and let $B$ be a connected component of the critical set $C r(f)$. For any $p \in B$ there is a local chart of $M$ around $p$ and a local splitting of the normal bundle of $B$,

$$
\nu_{*}(B)=\nu_{*}^{+}(B) \oplus \nu_{*}^{-}(B),
$$

identifying a point $x \in M$ in its domain to $(u, v, w)$, where $u \in B, v \in \nu_{*}^{+}(B)$, and $w \in \nu_{*}^{-}(B)$ such that within this chart $f$ assumes the form

$$
f(x)=f(u, v, w)=f(B)+|v|^{2}-|w|^{2} .
$$


Note that the Morse-Bott Lemma shows that if $B$ is connected, then $\lambda_{p}$ is independent of $p \in B$. Hence, we may also refer to $\lambda_{p}$ as the index $\lambda_{B}$ of the critical submanifold $B$. Moreover, the lemma shows that at a critical point $p \in \operatorname{Cr}(f)$ the tangent space splits as

$$
T_{p} M=T_{p} B \oplus \nu_{p}^{+}(B) \oplus \nu_{p}^{-}(B),
$$

where $\lambda_{p}=\operatorname{dim} \nu_{p}^{-}(B)$. If we let $b=\operatorname{dim} B$ and $\lambda_{p}^{*}=\operatorname{dim} \nu_{p}^{+}(B)$, then we have the fundamental relation

$$
m=b+\lambda_{p}^{*}+\lambda_{p}
$$

where $m=\operatorname{dim} M$.

Let $\varphi_{t}: M \rightarrow M$ denote the flow associated to $-\nabla f$, i.e. $\varphi_{t}(x)=\gamma(t)$, where $\gamma^{\prime}(t)=-(\nabla f)(\gamma(t))$ and $\gamma(0)=x$. For $p \in C r(f)$ the stable manifold $W^{s}(p)$ and the unstable manifold $W^{u}(p)$ are defined the same as they were for a Morse function:

$$
\begin{aligned}
W^{s}(p) & =\left\{x \in M \mid \lim _{t \rightarrow \infty} \varphi_{t}(x)=p\right\}, \\
W^{u}(p) & =\left\{x \in M \mid \lim _{t \rightarrow-\infty} \varphi_{t}(x)=p\right\} .
\end{aligned}
$$

However, for a Morse-Bott function we can also consider the stable and unstable manifolds of a critical submanifold $B \subseteq C r(f)$. These are defined to be

$$
\begin{aligned}
& W^{s}(B)=\bigcup_{p \in B} W^{s}(p), \\
& W^{u}(B)=\bigcup_{p \in B} W^{u}(p) .
\end{aligned}
$$

For the following see Proposition 3.2 of [4].

Theorem 3.3 (Stable/Unstable Manifold Theorem for a Morse-Bott function). The stable and unstable manifolds $W^{s}(B)$ and $W^{u}(B)$ are the surjective images of smooth injective immersions $E^{+}: \nu_{*}^{+}(B) \rightarrow M$ and $E^{-}: \nu_{*}^{-}(B) \rightarrow M$. There are smooth endpoint maps $\partial_{+}: W^{s}(B) \rightarrow B$ and $\partial_{-}: W^{u}(B) \rightarrow B$ given by $\partial_{+}(x)=\lim _{t \rightarrow \infty} \varphi_{t}(x)$ and $\partial_{-}(x)=\lim _{t \rightarrow-\infty} \varphi_{t}(x)$, which when restricted to a neighborhood of $B$ have the structure of locally trivial fiber bundles.

Definition 3.4 (Morse-Bott-Smale transversality). A Morse-Bott function $f$ : $M \rightarrow \mathbb{R}$ is said to satisfy the Morse-Bott-Smale transversality condition with respect to a given metric on $M$ if and only if for any two connected critical submanifolds $B$ and $B^{\prime}, W^{u}(p)$ intersects $W^{s}\left(B^{\prime}\right)$ transversely, i.e. $W^{u}(p) \pitchfork W^{s}\left(B^{\prime}\right)$, for all $p \in B$.

Note. By the Kupka-Smale Theorem (Theorem 2.1) we can always find a MorseSmale function as close as we like to a given Morse-Bott function. However, there are situations where a certain Morse-Bott function has some desired properties that will be lost if it is perturbed. In those cases it might be preferable to look for a Riemannian metric such that the function is Morse-Bott-Smale with respect to the chosen metric. Unfortunately, it is not always possible to perturb the Riemannian 
metric to make a given Morse-Bott function satisfy the Morse-Bott-Smale transversality condition. See Section 2 of [32] for some interesting counterexamples.

If the gradient flow of $f: M \rightarrow \mathbb{R}$ does satisfy the Morse-Bott-Smale transversality condition, then the space

$$
W\left(B, B^{\prime}\right)=W^{u}(B) \cap W^{s}\left(B^{\prime}\right)
$$

of points $x \in M$ such that $\partial_{-}(x) \in B$ and $\partial_{+}(x) \in B^{\prime}$ is a submanifold of $M$. The following lemma is an immediate consequence of transversality.

Lemma 3.5. Suppose that $B$ is of dimension $b$ and index $\lambda_{B}$ and that $B^{\prime}$ is of dimension $b^{\prime}$ and index $\lambda_{B^{\prime}}$. Then we have the following, where $m=\operatorname{dim} M$ :

$$
\begin{aligned}
\operatorname{dim} W^{u}(B) & =b+\lambda_{B}, \\
\operatorname{dim} W^{s}\left(B^{\prime}\right) & =b^{\prime}+\lambda_{B^{\prime}}^{*}=m-\lambda_{B^{\prime}}, \\
\operatorname{dim} W\left(B, B^{\prime}\right) & =\lambda_{B}-\lambda_{B^{\prime}}+b \quad\left(\text { if } W\left(B, B^{\prime}\right) \neq \emptyset\right) .
\end{aligned}
$$

Note that the dimension of $W\left(B, B^{\prime}\right)$ does not depend on the dimension of the critical submanifold $B^{\prime}$. This fact will be used when we define the boundary operator in the Morse-Bott-Smale chain complex.

We end this section with the following lemma, which says that a Morse-BottSmale function is weakly self-indexing 4, i.e. the Morse-Bott index is strictly decreasing along gradient flow lines.

Lemma 3.6. If $f: M \rightarrow \mathbb{R}$ satisfies the Morse-Bott-Smale transversality condition, then $W\left(B, B^{\prime}\right)=\emptyset$ whenever $\lambda_{B} \leq \lambda_{B^{\prime}}$ and $B \neq B^{\prime}$.

Proof. If $B \neq B^{\prime}$ and there exists an $x \in W\left(B, B^{\prime}\right)$, then $x$ lies on a one-dimensional gradient flow line that begins at $\partial_{-}(x) \in B$ and ends at $\partial_{+}(x) \in B^{\prime}$. Hence, $\operatorname{dim} W\left(\partial_{-}(x), B^{\prime}\right) \geq 1$, and the Morse-Bott-Smale transversality condition implies that $\operatorname{dim} W\left(\partial_{-}(x), B^{\prime}\right)=\lambda_{B}-\lambda_{B^{\prime}}+0 \geq 1$. Therefore, $\lambda_{B}>\lambda_{B^{\prime}}$.

\section{TOPOlOGical Chains AND FIBERED PRODUCTS}

In this section we introduce the concept of abstract topological chains and singular topological chains. Abstract topological chains are generalizations of the standard $p$-simplex $\Delta^{p} \subset \mathbb{R}^{p+1}$, and singular topological chains are generalizations of singular chains where we consider continuous maps from abstract topological chains. Applying these definitions to the faces of an $N$-cube, we construct a chain complex that is comparable to the singular cubical chain complex of 35. We also define the fibered product of singular topological chains and show that the fibered product of singular topological chains is an abstract topological chain. The homomorphism $\partial_{0}$ in the Morse-Bott-Smale chain complex constructed in Section [5 is defined in terms of abstract topological chains that are constructed from fibered products of compactified moduli spaces of gradient flow lines and the faces of an $N$-cube. In this section we define the degrees and boundary operators for these spaces and show that they satisfy the axioms for abstract topological chains.

4.1. Some general abstract definitions. For each integer $p \geq 0$ fix a set $C_{p}$ of topological spaces and let $S_{p}$ be the free abelian group generated by the elements of $C_{p}$, i.e. $S_{p}=\mathbb{Z}\left[C_{p}\right]$. Set $S_{p}=\{0\}$ if $p<0$ or $C_{p}=\emptyset$. 
Definition 4.1. A boundary operator on the collection $S_{*}$ of groups $\left\{S_{p}\right\}$ is a homomorphism $\partial_{p}: S_{p} \rightarrow S_{p-1}$ such that

(1) For $p \geq 1$ and $P \in C_{p} \subseteq S_{p}, \partial_{p}(P)=\sum_{k} n_{k} P_{k}$, where $n_{k}= \pm 1$ and $P_{k} \in C_{p-1}$ is a subspace of $P$ for all $k$.

(2) $\partial_{p-1} \circ \partial_{p}: S_{p} \rightarrow S_{p-2}$ is zero.

We will call $\left(S_{*}, \partial_{*}\right)$ a chain complex of abstract topological chains. Elements of $S_{p}$ are called abstract topological chains of degree $p$.

Definition 4.2. Let $B$ be a topological space and $p \in \mathbb{Z}_{+}$. A singular $C_{p}$-space in $B$ is a continuous map $\sigma: P \rightarrow B$ where $P \in C_{p}$, and the singular $C_{p}$-chain group $S_{p}(B)$ is the free abelian group generated by the singular $C_{p}$-spaces. Define $S_{p}(B)=\{0\}$ if $S_{p}=\{0\}$ or $B=\emptyset$. Elements of $S_{p}(B)$ are called singular topological chains of degree $p$.

For $p \geq 1$ there is a boundary operator $\partial_{p}: S_{p}(B) \rightarrow S_{p-1}(B)$ induced from the boundary operator $\partial_{p}: S_{p} \rightarrow S_{p-1}$. If $\sigma: P \rightarrow B$ is a singular $C_{p}$-space in $B$, then $\partial_{p}(\sigma)$ is given by the formula

$$
\partial_{p}(\sigma)=\left.\sum_{k} n_{k} \sigma\right|_{P_{k}}
$$

where

$$
\partial_{p}(P)=\sum_{k} n_{k} P_{k}
$$

The pair $\left(S_{*}(B), \partial_{*}\right)$ is called a chain complex of singular topological chains in $B$.

Note. The preceding definitions are quite general. To construct the Morse-BottSmale chain complex in Section [5 we only need $C_{p}$ to include the $p$-dimensional faces of an $N$-cube, the compactified moduli spaces of gradient flow lines of dimension $p$, and the components of their fibered products of dimension $p$. In this section we show that these spaces carry the structure of abstract topological chains.

4.2. Singular $N$-cube chains. In this subsection we show how the above abstract definitions apply to the faces of an $N$-cube to produce a chain complex comparable to the singular cubical chain complex of [35].

Pick some large positive integer $N$ and let $I^{N}=\left\{\left(x_{1}, \ldots, x_{N}\right) \in \mathbb{R}^{N} \mid 0 \leq x_{j} \leq\right.$ $1, j=1, \ldots, N\}$ denote the unit $N$-cube. For every $0 \leq p \leq N$ let $C_{p}$ be the set consisting of the faces of $I^{N}$ of dimension $p$, i.e. subsets of $I^{N}$ where $p$ of the coordinates are free and the rest of the coordinates are fixed to be either 0 or 1 . For every $0 \leq p \leq N$ let $S_{p}$ be the free abelian group generated by the elements of $C_{p}$.

For $P \in C_{p}$ we define

$$
\partial_{p}(P)=\sum_{j=1}^{p}(-1)^{j}\left[\left.P\right|_{x_{j}=1}-\left.P\right|_{x_{j}=0}\right] \in S_{p-1},
$$

where $x_{j}$ denotes the $j^{\text {th }}$ free coordinate of $P$. The boundary operator $\partial_{p}$ extends linearly to a homomorphism $\partial_{p}: S_{p} \rightarrow S_{p-1}$. To see that $\partial_{p-1} \circ \partial_{p}=0$ note that 
in the sum for $\partial_{p-1}\left(\partial_{p}(P)\right)$,

$$
\sum_{i=1}^{p-1}(-1)^{i}\left[\left.\sum_{j=1}^{p}(-1)^{j}\left[\left.P\right|_{x_{j}=1}-\left.P\right|_{x_{j}=0}\right]\right|_{\tilde{x}_{i}=1}-\left.\sum_{j=1}^{p}(-1)^{j}\left[\left.P\right|_{x_{j}=1}-\left.P\right|_{x_{j}=0}\right]\right|_{\tilde{x}_{i}=0}\right],
$$

the terms cancel in pairs, and hence $\partial_{p-1}\left(\partial_{p}(P)\right)=0 \in S_{p-2}$. A continuous map $\sigma_{P}: P \rightarrow B$ into a topological space $B$ is a singular $C_{p}$-space in $B$. The boundary operator applied to $\sigma_{P}$ is

$$
\partial_{p}\left(\sigma_{P}\right)=\sum_{j=1}^{p}(-1)^{j}\left[\left.\sigma_{P}\right|_{x_{j}=1}-\left.\sigma_{P}\right|_{x_{j}=0}\right] \in S_{p-1}(B),
$$

where $\left.\sigma_{P}\right|_{x_{j}=0}$ denotes the restriction $\sigma_{P}:\left.P\right|_{x_{j}=0} \rightarrow B$ and $\left.\sigma_{P}\right|_{x_{j}=1}$ denotes the restriction $\sigma_{P}:\left.P\right|_{x_{j}=1} \rightarrow B$. We will show that the $p^{\text {th }}$ singular homology group of $B$ is isomorphic to the homology of a quotient of the chain complex $\left(S_{*}(B), \partial_{*}\right)$ by the degenerate singular chains for $p<N$.

Definition 4.3 (Degeneracy relations for singular $N$-cube chains). Let $\sigma_{P}$ and $\sigma_{Q}$ be singular $C_{p^{-}}$-spaces in $B$ and let $\partial_{p}(Q)=\sum_{j} n_{j} Q_{j} \in S_{p-1}$. For any map $\alpha: P \rightarrow Q$, let $\partial_{p}\left(\sigma_{Q}\right) \circ \alpha$ denote the formal sum $\left.\sum_{j} n_{j}\left(\sigma_{Q} \circ \alpha\right)\right|_{\alpha^{-1}\left(Q_{j}\right)}$. Define the subgroup $D_{p}(B) \subseteq S_{p}(B)$ of degenerate singular $N$-cube chains to be the subgroup generated by the following elements:

(1) If $\alpha$ is an orientation preserving homeomorphism such that $\sigma_{Q} \circ \alpha=\sigma_{P}$ and $\partial_{p}\left(\sigma_{Q}\right) \circ \alpha=\partial_{p}\left(\sigma_{P}\right)$, then $\sigma_{P}-\sigma_{Q} \in D_{p}(B)$.

(2) If $\sigma_{P}$ does not depend on some free coordinate of $P$, then $\sigma_{P} \in D_{p}(B)$.

Note. We orient the faces of $I^{N}$ of dimension $p$ by requiring that the map which identifies the free coordinates $\left(x_{1}, \ldots, x_{p}\right)$ of a face with the standard coordinates of $\mathbb{R}^{p}$ be orientation preserving. Also, a map from a vertex to a vertex is orientation preserving.

Theorem 4.4 (Singular $N$-cube chain theorem). The boundary operator for singular $N$-cube chains $\partial_{p}: S_{p}(B) \rightarrow S_{p-1}(B)$ descends to a homomorphism

$$
\partial_{p}: S_{p}(B) / D_{p}(B) \rightarrow S_{p-1}(B) / D_{p-1}(B),
$$

and

$$
H_{p}\left(S_{*}(B) / D_{*}(B), \partial_{*}\right) \approx H_{p}(B ; \mathbb{Z})
$$

for all $p<N$.

Proof. Let $\sigma_{P} \in S_{p}(B)$ be a singular $C_{p}$-space and assume that there exists a singular $C_{p}$-space $\sigma_{Q} \in S_{p}(B)$ and an orientation preserving homeomorphism $\alpha$ : $P \rightarrow Q$ such that $\sigma_{Q} \circ \alpha=\sigma_{P}$ and $\partial_{p}\left(\sigma_{Q}\right) \circ \alpha=\partial_{p}\left(\sigma_{P}\right)$. Since $\partial_{p-1}\left(\partial_{p}\left(\sigma_{Q}\right)\right)=$ $\partial_{p-1}\left(\partial_{p}\left(\sigma_{P}\right)\right)=0$, we have $\partial_{p-1}\left(\partial_{p}\left(\sigma_{Q}\right)\right) \circ \alpha=\partial_{p-1}\left(\partial_{p}\left(\sigma_{P}\right)\right)$. Thus, $\partial_{p}\left(\sigma_{P}-\sigma_{Q}\right)=$ $\partial_{p}\left(\sigma_{P}\right)-\partial_{p}\left(\sigma_{Q}\right) \in D_{p-1}(B)$.

Now assume that $\sigma_{P} \in S_{p}(B)$ is a singular $C_{p}$-space that does not depend on some free coordinate $x_{j}$. Then in the sum for $\partial_{p}\left(\sigma_{P}\right)$ the term $\left.\sigma_{P}\right|_{x_{j}=1}-\left.\sigma_{P}\right|_{x_{j}=0} \in$ $D_{p-1}(B)$ because $\left.\sigma_{P}\right|_{x_{j}=0}$ and $\left.\sigma_{P}\right|_{x_{j}=1}$ satisfy the first condition for degeneracy, and the rest of the terms are all independent of the coordinate corresponding to $x_{j}$ and thus satisfy the second condition for degeneracy. Therefore $\partial_{p}\left(D_{p}(B)\right) \subseteq D_{p-1}(B)$, and the boundary operator $\partial_{p}$ descends to a homomorphism of the quotient groups. 
We now recall the definition of singular homology using singular cubes in 35 . Let $I^{p}$ be the standard unit $p$-cube for $p>0$ and let $I^{0}$ be the origin in $\mathbb{R}^{N}$. We will view $I^{p} \in C_{p}$ by identifying $I^{p}$ with $I^{p} \times(0, \ldots, 0) \subseteq I^{N}$. A singular $p$-cube in $B$ is a continuous map $T: I^{p} \rightarrow B$, i.e. a singular $C_{p}$-space in $B$ with domain $I^{p}$. Let $Q_{p}(B) \subseteq S_{p}(B)$ denote the free abelian group generated by the set of all singular $p$-cubes in $B$. A singular $p$-cube is called degenerate if it satisfies the second condition listed in Definition 4.3. Note that the first condition is not needed to define singular homology using singular $p$-cubes because all singular $p$-cubes are defined on the same domain. Let $D Q_{p}(B)$ denote the subgroup of $Q_{p}(B)$ generated by the degenerate singular $p$-cubes.

If $T: I^{p} \rightarrow B$ is a singular $p$-cube, then for $i=1, \ldots, p$ there are singular $p-1$ cubes defined by the formulas

$$
\begin{gathered}
A_{i} T\left(x_{1}, \ldots, x_{p-1}\right)=T\left(x_{1}, \ldots, x_{i-1}, 1, x_{i}, \ldots, x_{p-1}\right) \\
B_{i} T\left(x_{1}, \ldots, x_{p-1}\right)=T\left(x_{1}, \ldots, x_{i-1}, 0, x_{i}, \ldots, x_{p-1}\right)
\end{gathered}
$$

and a boundary operator defined by

$$
\tilde{\partial}_{p}(T)=\sum_{i=1}^{p}(-1)^{i}\left[A_{i} T-B_{i} T\right]
$$

The boundary operator satisfies $\tilde{\partial}_{p}\left(D Q_{p}(B)\right) \subseteq D Q_{p-1}(B)$, and the singular homology of $B$ is the homology of the chain complex $\left(Q_{*}(B) / D Q_{*}(B), \tilde{\partial}_{*}\right)$. For more details see Chapter VII of [35].

Now assume that $p<N$. Since $Q_{p}(B) \subseteq S_{p}(B)$ and $D Q_{p}(B) \subseteq D_{p}(B)$ there are induced maps such that

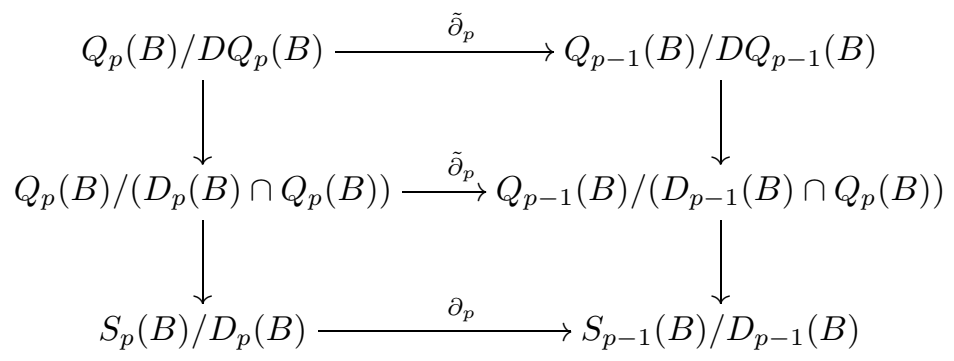

commutes for all $p$. We will show that the induced maps in homology are isomorphisms. To see that this is true for the bottom square, note that for any $P \in C_{p}$ there is an orientation preserving homeomorphism $\alpha^{-1}: I^{p} \rightarrow P$ defined by sending the fixed coordinates of $I^{p} \times(0, \ldots, 0) \subseteq I^{N}$ to the fixed coordinates of $P$ and the $j^{\text {th }}$ free coordinate of $I^{p}$ to the $j^{\text {th }}$ free coordinate of $P$ for all $j=1, \ldots, p$. For any singular $C_{p}$-space $\sigma_{P} \in S_{p}(B), \sigma_{Q}=\sigma_{P} \circ \alpha^{-1}: I^{p} \rightarrow B$ is a singular $C_{p}$-space that satisfies $\sigma_{Q} \circ \alpha=\sigma_{P}$ and $\partial_{p}\left(\sigma_{Q}\right) \circ \alpha=\partial_{p}\left(\sigma_{P}\right)$. Therefore, $\left[\sigma_{P}\right]=\left[\sigma_{Q}\right] \in S_{p}(B) / D_{p}(B)$, and we see that every equivalence class in $S_{p}(B) / D_{p}(B)$ has a representative that is defined on the standard unit $p$-cube $I^{p}$. Therefore, $S_{p}(B) / D_{p}(B) \approx Q_{p}(B) /\left(D_{p}(B) \cap Q_{p}(B)\right)$ for all $p$, and the homology groups of $\left(Q_{*}(B) /\left(D_{*}(B) \cap Q_{*}(B)\right), \tilde{\partial}_{*}\right)$ are isomorphic to the homology groups of $\left(S_{*}(B) / D_{*}(B), \partial_{*}\right)$. 
To show that the top square in the above diagram induces isomorphisms of the homology groups, we will show that the homology groups of the chain complex

$$
\left(\left(D_{*}(B) \cap Q_{*}(B)\right) / D Q_{*}(B), \tilde{\partial}_{*}\right)
$$

are zero for all $p$. Thus, the long exact sequence induced from

$0 \rightarrow\left(D_{*}(B) \cap Q_{*}(B)\right) / D Q_{*}(B) \rightarrow Q_{*}(B) / D Q_{*}(B) \rightarrow Q_{*}(B) /\left(D_{*}(B) \cap Q_{*}(B)\right) \rightarrow 0$

shows that the homology groups of $\left(Q_{*}(B) /\left(D_{*}(B) \cap Q_{*}(B)\right), \tilde{\partial}_{*}\right)$ are isomorphic to the homology groups of $\left(Q_{*}(B) / D Q_{*}(B), \tilde{\partial}_{*}\right)$.

Let $G_{p}=\left(D_{p}(B) \cap Q_{p}(B)\right) / D Q_{p}(B)$. Note that $G_{0}=\{0\}$ since $D_{0}(B) \cap Q_{0}(B)=$ $\{0\}$. Thus, $\tilde{\partial}_{1}$ is surjective and $H_{0}\left(G_{*}, \tilde{\partial}_{*}\right)=\{0\}$. Now assume that $p>0$ and let

$$
\sum_{k} n_{k}\left(\sigma_{k} \circ \alpha_{k}-\sigma_{k}\right) \in D_{p}(B) \cap Q_{p}(B)
$$

be a representative for an element of $\operatorname{ker}\left(\tilde{\partial}_{p}\right) \subseteq G_{p}$, i.e.

$$
\tilde{\partial}_{p}\left(\sum_{k} n_{k}\left(\sigma_{k} \circ \alpha_{k}-\sigma_{k}\right)\right)=0,
$$

and for every $k$ we have $\partial_{p}\left(\sigma_{k}\right) \circ \alpha_{k}=\partial_{p}\left(\sigma_{k} \circ \alpha_{k}\right)$, where $\alpha_{k}: I^{p} \rightarrow I^{p}$ is an orientation preserving homeomorphism. Extend each $\alpha_{k}$ to an orientation preserving homeomorphism $\tilde{\alpha}_{k}: I^{p+1} \rightarrow I^{p+1}$ by the formula $\tilde{\alpha}_{k}\left(x_{1}, \ldots, x_{p}, x_{p+1}\right)=$ $\left(\alpha_{k}\left(x_{1}, \ldots, x_{p}\right), x_{p+1}\right)$, and extend each $\sigma_{k}: I^{p} \rightarrow B$ to a map $\tilde{\sigma}_{k}: I^{p+1} \rightarrow B$ such that for every $0 \leq x_{p+1} \leq 1$ we have

$$
\partial_{p}\left(\sum_{k} n_{k}\left(\tilde{\sigma}_{k}\left(-, x_{p+1}\right)\right) \circ \tilde{\alpha}_{k}-\tilde{\sigma}_{k}\left(-, x_{p+1}\right)\right)=0
$$

$\partial_{p}\left(\tilde{\sigma}_{k}\left(-, x_{p+1}\right)\right) \circ \tilde{\alpha}_{k}=\partial_{p}\left(\tilde{\sigma}_{k} \circ \tilde{\alpha}_{k}\left(-, x_{p+1}\right)\right)$, and each $\tilde{\sigma}_{k}$ is constant when $x_{p+1}=1$. Then

$$
\sum_{k} n_{k}\left(\tilde{\sigma}_{k} \circ \tilde{\alpha}_{k}-\tilde{\sigma}_{k}\right) \in D_{p+1}(B) \cap Q_{p+1}(B)
$$

satisfies

$$
\tilde{\partial}_{p+1}\left[\sum_{k} n_{k}\left(\tilde{\sigma}_{k} \circ \tilde{\alpha}_{k}-\tilde{\sigma}_{k}\right)\right]=(-1)^{p+1}\left[\sum_{k} n_{k}\left(\sigma_{k} \circ \alpha_{k}-\sigma_{k}\right)\right]
$$

in $\left(D_{p}(B) \cap Q_{p}(B)\right) / D Q_{p}(B)$. Therefore, $\tilde{\partial}_{p+1}$ maps onto $\operatorname{ker}\left(\tilde{\partial}_{p}\right)$ and $\left(G_{*}, \tilde{\partial}_{*}\right)$ is acyclic.

4.3. The fibered product of singular topological chains. In this subsection we resume the discussion of our abstract definitions. We define the fibered product of singular topological chains and show that the fibered product of singular topological chains is an abstract topological chain.

Let $\sigma_{i}: P_{i} \rightarrow B$ for $i=1,2$ be two continuous maps into a topological space $B$. Recall that the fibered product of $\sigma_{1}$ and $\sigma_{2}$ is defined to be

$$
P_{1} \times{ }_{B} P_{2}=\left(\sigma_{1} \times \sigma_{2}\right)^{-1}(\Delta),
$$

where $\Delta$ is the diagonal in $B \times B$, i.e.

$$
P_{1} \times_{B} P_{2}=\left\{\left(x_{1}, x_{2}\right) \in P_{1} \times P_{2} \mid \sigma_{1}\left(x_{1}\right)=\sigma_{2}\left(x_{2}\right)\right\} .
$$


Lemma 4.5. Suppose that $\sigma_{1}: P_{1} \rightarrow B$ and $\sigma_{2}: P_{2} \rightarrow B$ are smooth maps, where $P_{1}, P_{2}$, and $B$ are smooth manifolds of dimension $p_{1}, p_{2}$, and $b$ respectively. If $\sigma_{1}$ is transverse to $\sigma_{2}$, then the fibered product $P_{1} \times_{B} P_{2}$ is a smooth manifold of dimension $p_{1}+p_{2}-b$.

Proof. This follows from the fact that $\sigma_{1} \pitchfork \sigma_{2}$ if and only if $\left(\sigma_{1} \times \sigma_{2}\right) \pitchfork \Delta$.

Given a collection of topological spaces $\left\{C_{p}\right\}_{p \geq 0}$ we will say that an element $P \in C_{p}$ has degree $p$. If $B$ is a topological manifold of dimension $b, P_{1} \in C_{p_{1}}$, $P_{2} \in C_{p_{2}}$, and $\sigma_{i}: P_{i} \rightarrow B$ for $i=1,2$ are continuous maps, then we can associate the degree $p_{1}+p_{2}-b$ to the fibered product $P_{1} \times_{B} P_{2}$ using the same formula as above. If we assume that the collection of spaces $\left\{C_{p}\right\}_{p \geq 0}$ is closed under the fibered product construction with respect to some collection of maps, i.e. if

$$
P_{1} \times{ }_{B} P_{2} \in C_{p_{1}+p_{2}-b},
$$

then the fibered product construction extends linearly to the collection of free abelian groups $\left\{S_{p}\right\}$.

Definition 4.6. Suppose that $\left\{C_{p}\right\}_{p \geq 0}$ is a collection of topological spaces that is closed under the fibered product construction with respect to some collection of maps, and assume that $\left(S_{*}, \partial_{*}\right)$ is a chain complex of abstract topological chains based on the collection $\left\{C_{p}\right\}_{p \geq 0}$. If $\sigma_{i}=\sum_{k} n_{i, k} \sigma_{i, k} \in S_{p_{i}}(B)$ for $i=1,2$, where $\sigma_{i, k}: P_{i, k} \rightarrow B$ is a singular $\bar{C}_{p_{i}}$-space for all $k$, then the fibered product of $\sigma_{1}$ and $\sigma_{2}$ over $B$ is defined to be

$$
P_{1} \times_{B} P_{2}=\sum_{k, j} n_{1, k} n_{2, j} P_{1, k} \times{ }_{B} P_{2, j},
$$

where $P_{1}=\sum_{k} n_{1, k} P_{1, k} \in S_{p_{1}}$ and $P_{2}=\sum_{j} n_{2, j} P_{2, j} \in S_{p_{2}}$. The boundary operator applied to the fibered product is defined to be

$$
\partial\left(P_{1} \times_{B} P_{2}\right)=\partial P_{1} \times_{B} P_{2}+(-1)^{p_{1}+b} P_{1} \times_{B} \partial P_{2} .
$$

If $\sigma_{i}=0$ for either $i=1$ or 2 , then we define $P_{1} \times_{B} P_{2}=0$.

Note. We omit the subscript $p$ on the boundary operator $\partial_{p}$ in order to simplify the notation. Also, given the data of a triple

$$
P_{1} \stackrel{\sigma_{11}}{\longrightarrow} B_{1} \stackrel{\sigma_{12}}{\longleftarrow} P_{2} \stackrel{\sigma_{22}}{\longrightarrow} B_{2} \stackrel{\sigma_{23}}{\longleftarrow} P_{3}
$$

we can form the iterated fibered product $\left(P_{1} \times{ }_{B_{1}} P_{2}\right) \times{ }_{B_{2}} P_{3}$ using $\sigma_{23}$ and the map $\sigma_{22} \circ \pi_{2}: P_{1} \times{ }_{B_{1}} P_{2} \rightarrow B_{2}$, where $\pi_{2}: P_{1} \times{ }_{B_{1}} P_{2} \rightarrow P_{2}$ denotes projection to the second component. That is, we have the following diagram:

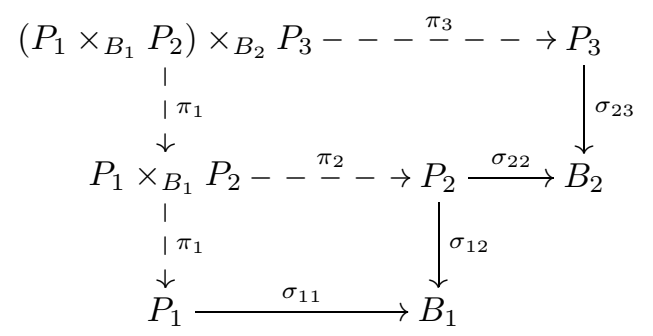

Similarly, we can form the iterated fibered product $P_{1} \times{ }_{B_{1}}\left(P_{2} \times_{B_{2}} P_{3}\right)$ using $\sigma_{11}$ and the map $\sigma_{12} \circ \pi_{1}: P_{1} \times_{B_{1}} P_{2} \rightarrow B_{1}$, where $\pi_{1}: P_{2} \times_{B_{2}} P_{3} \rightarrow P_{2}$ denotes 
projection to the first component. This corresponds to the following diagram:

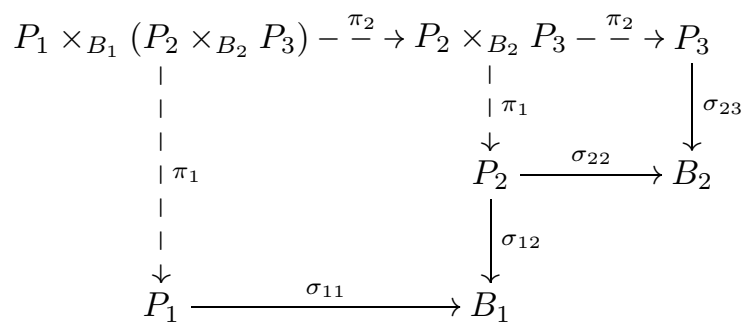

It is easy to see that $\left(P_{1} \times_{B_{1}} P_{2}\right) \times_{B_{2}} P_{3}$ and $P_{1} \times_{B_{1}}\left(P_{2} \times_{B_{2}} P_{3}\right)$ are the same as topological spaces. The following lemma shows that they are also the same as abstract topological chains.

Lemma 4.7. The fibered product of two singular topological chains is an abstract topological chain; i.e. the boundary operator on fibered products is of degree -1 and satisfies $\partial \circ \partial=0$. Moreover, the boundary operator on fibered products is associative; i.e.

$$
\partial\left(\left(P_{1} \times_{B_{1}} P_{2}\right) \times_{B_{2}} P_{3}\right)=\partial\left(P_{1} \times_{B_{1}}\left(P_{2} \times_{B_{2}} P_{3}\right)\right) .
$$

Proof. Since $\partial$ is a boundary operator on $P_{1}$ and $P_{2}$, the degree of $\partial P_{1}$ is $p_{1}-1$ and the degree of $\partial P_{2}$ is $p_{2}-1$. Hence both $\partial P_{1} \times_{B} P_{2}$ and $P_{1} \times_{B} \partial P_{2}$ have degree $p_{1}+p_{2}-b-1$.

To see that $\partial^{2}\left(P_{1} \times{ }_{B} P_{2}\right)=0$ we compute as follows:

$$
\begin{aligned}
\partial\left(\partial\left(P_{1} \times{ }_{B} P_{2}\right)\right)= & \partial\left(\partial P_{1} \times{ }_{B} P_{2}+(-1)^{p_{1}+b} P_{1} \times{ }_{B} \partial P_{2}\right) \\
= & \partial^{2} P_{1} \times{ }_{B} P_{2}+(-1)^{p_{1}-1+b} \partial P_{1} \times{ }_{B} \partial P_{2} \\
& \quad+(-1)^{p_{1}+b}\left(\partial P_{1} \times{ }_{B} \partial P_{2}+(-1)^{p_{1}+b} P_{1} \times{ }_{B} \partial^{2} P_{2}\right) \\
= & 0 .
\end{aligned}
$$

To prove associativity we compute as follows:

$$
\begin{aligned}
\partial( & \left.P_{1} \times_{B_{1}}\left(P_{2} \times_{B_{2}} P_{3}\right)\right) \\
= & \partial P_{1} \times_{B_{1}}\left(P_{2} \times_{B_{2}} P_{3}\right)+(-1)^{p_{1}+b_{1}} P_{1} \times_{B_{1}} \partial\left(P_{2} \times_{B_{2}} P_{3}\right) \\
= & \partial P_{1} \times_{B_{1}}\left(P_{2} \times_{B_{2}} P_{3}\right) \\
& +(-1)^{p_{1}+b_{1}}\left(P_{1} \times_{B_{1}}\left(\partial P_{2} \times_{B_{2}} P_{3}+(-1)^{p_{2}+b_{2}} P_{2} \times_{B_{2}} \partial P_{3}\right)\right) \\
= & \partial P_{1} \times_{B_{1}} P_{2} \times_{B_{2}} P_{3}+(-1)^{p_{1}+b_{1}} P_{1} \times_{B_{1}} \partial P_{2} \times_{B_{2}} P_{3} \\
& +(-1)^{p_{1}+p_{2}+b_{1}+b_{2}} P_{1} \times_{B_{1}} P_{2} \times_{B_{2}} \times \partial P_{3}, \\
\partial( & \left.\left(P_{1} \times_{B_{1}} P_{2}\right) \times_{B_{2}} P_{3}\right) \\
= & \partial\left(P_{1} \times_{B_{1}} P_{2}\right) \times_{B_{2}} P_{3}+(-1)^{\operatorname{deg}\left(P_{1} \times_{B_{1}} P_{2}\right)+b_{2}}\left(P_{1} \times_{B_{1}} P_{2}\right) \times_{B_{2}} \partial P_{3} \\
= & \left(\partial P_{1} \times_{B_{1}} P_{2}+(-1)^{p_{1}+b_{1}} P_{1} \times_{B_{1}} \partial P_{2}\right) \times_{B_{2}} P_{3} \\
& +(-1)^{p_{1}+p_{2}-b_{1}+b_{2}} P_{1} \times_{B_{1}} P_{2} \times_{B_{2}} \partial P_{3} \\
= & \partial P_{1} \times_{B_{1}} P_{2} \times_{B_{2}} P_{3}+(-1)^{p_{1}+b_{1}} P_{1} \times_{B_{1}} \partial P_{2} \times_{B_{2}} P_{3} \\
& +(-1)^{p_{1}+p_{2}-b_{1}+b_{2}} P_{1} \times{ }_{B_{1}} P_{2} \times_{B_{2}} \times \partial P_{3} .
\end{aligned}
$$


4.4. Compactified moduli spaces as singular topological chains. In this subsection we define a degree and boundary operator for compactified moduli spaces of gradient flow lines. We will show in Subsection 5.5 that these compactified moduli spaces are smooth manifolds with corners and that the degree defined in this subsection coincides with the dimension of these manifolds. The abstract topological chain structure defined in this subsection, together with those defined for the faces of an $N$-cube and for fibered products, are used in Section 5 to define the homomorphism $\partial_{0}$ in the Morse-Bott-Smale chain complex.

Let $f: M \rightarrow \mathbb{R}$ be a Morse-Bott function on a Riemannian manifold $M$ and let $\varphi_{t}: M \rightarrow M$ denote the flow associated to $-\nabla f$. For any two critical submanifolds $B$ and $B^{\prime}$, the flow $\varphi_{t}$ induces an $\mathbb{R}$-action on $W^{u}(B) \cap W^{s}\left(B^{\prime}\right)$. Let

$$
\mathcal{M}\left(B, B^{\prime}\right)=\left(W^{u}(B) \cap W^{s}\left(B^{\prime}\right)\right) / \mathbb{R}
$$

be the quotient space of gradient flow lines from $B$ to $B^{\prime}$. For proofs of the following two fundamental theorems we refer the reader to Appendix $\S$ A.3 of 4 (see [13, [27, and 26] for a different approach to Theorem 4.9).

Theorem 4.8 (Gluing). Suppose that $B, B^{\prime}$, and $B^{\prime \prime}$ are critical submanifolds such that $W^{u}(B) \pitchfork W^{s}\left(B^{\prime}\right)$ and $W^{u}\left(B^{\prime}\right) \pitchfork W^{s}\left(B^{\prime \prime}\right)$. In addition, assume that $W^{u}(x) \pitchfork W^{s}\left(B^{\prime \prime}\right)$ for all $x \in B^{\prime}$. Then for some $\epsilon>0$, there is an injective local diffeomorphism

$$
G: \mathcal{M}\left(B, B^{\prime}\right) \times{ }_{B^{\prime}} \mathcal{M}\left(B^{\prime}, B^{\prime \prime}\right) \times(0, \epsilon) \rightarrow \mathcal{M}\left(B, B^{\prime \prime}\right)
$$

onto an end of $\mathcal{M}\left(B, B^{\prime \prime}\right)$.

Theorem 4.9 (Compactification). Assume that $f: M \rightarrow \mathbb{R}$ satisfies the MorseBott-Smale transversality condition. For any two distinct connected critical submanifolds $B$ and $B^{\prime}$, the moduli space $\mathcal{M}\left(B, B^{\prime}\right)$ has a compactification $\overline{\mathcal{M}}\left(B, B^{\prime}\right)$, consisting of all the piecewise gradient flow lines from $B$ to $B^{\prime}$, which is either empty or a compact smooth manifold with corners of dimension $\lambda_{B}-\lambda_{B^{\prime}}+b-1$. Moreover, the beginning and endpoint maps extend to smooth maps

$$
\begin{aligned}
& \partial_{-}: \overline{\mathcal{M}}\left(B, B^{\prime}\right) \rightarrow B, \\
& \partial_{+}: \overline{\mathcal{M}}\left(B, B^{\prime}\right) \rightarrow B^{\prime},
\end{aligned}
$$

where the beginning point map $\partial_{-}$has the structure of a locally trivial fiber bundle.

The compactified moduli spaces of gradient flow lines for a Morse-Bott-Smale function can be described inductively as follows. For any two critical submanifolds $B$ and $B^{\prime}$, define $B \succ B^{\prime}$ if $B \neq B^{\prime}$ and there exists a gradient flow line from $B$ to $B^{\prime}$. Note that this relationship is transitive; i.e. if $B \succ B^{\prime}$ and $B^{\prime} \succ B^{\prime \prime}$, then $B \succ B^{\prime \prime}$ by Theorem 4.8. If there is no critical submanifold $B^{\prime}$ such that $B \succ B^{\prime} \succ B^{\prime \prime}$, then $\overline{\mathcal{M}}\left(B, B^{\prime \prime}\right)=\mathcal{M}\left(B, B^{\prime \prime}\right)$. In general,

$$
\overline{\mathcal{M}}\left(B, B^{\prime \prime}\right)=\mathcal{M}\left(B, B^{\prime \prime}\right) \cup \bigcup_{B \succ B^{\prime} \succ B^{\prime \prime}} \overline{\mathcal{M}}\left(B, B^{\prime}\right) \times_{B^{\prime}} \overline{\mathcal{M}}\left(B^{\prime}, B^{\prime \prime}\right),
$$

where the union is taken over all critical submanifolds $B^{\prime}$ between $B$ and $B^{\prime \prime}$. Note that this description is inductive; i.e. both $\overline{\mathcal{M}}\left(B, B^{\prime}\right)$ and $\overline{\mathcal{M}}\left(B^{\prime}, B^{\prime \prime}\right)$ may contain fibered products of compactified moduli spaces of smaller dimension. Hence $\overline{\mathcal{M}}\left(B, B^{\prime \prime}\right)$ consists of all piecewise gradient flow lines from $B$ to $B^{\prime \prime}$. The topology of $\overline{\mathcal{M}}\left(B, B^{\prime}\right)$ is determined by considering its elements as one-dimensional subsets of $M$. 
Definition 4.10. The topology on $\overline{\mathcal{M}}\left(B, B^{\prime}\right)$ is the topology induced from the Hausdorff metric $d_{H}$ on subsets of $M$. That is, if $l_{1}, l_{2} \in \overline{\mathcal{M}}\left(B, B^{\prime}\right)$, then $l_{1}$ and $l_{2}$ determine subsets $l_{1}, l_{2} \subseteq M$, which are images of continuous injective paths from $B$ to $B^{\prime}$, and

$$
d_{H}\left(l_{1}, l_{2}\right)=\sup _{x_{1} \in l_{1}} \inf _{x_{2} \in l_{2}} d\left(x_{1}, x_{2}\right)+\sup _{x_{2} \in l_{2}} \inf _{x_{1} \in l_{1}} d\left(x_{1}, x_{2}\right),
$$

where $d$ is the metric on $M$.

Note that this definition is compatible with the topology of fibered products. That is, if $B \succ B^{\prime} \succ B^{\prime \prime}$, then $\overline{\mathcal{M}}\left(B, B^{\prime}\right) \times_{B^{\prime}} \overline{\mathcal{M}}\left(B^{\prime}, B^{\prime \prime}\right)$ inherits a topology as a subspace of $\overline{\mathcal{M}}\left(B, B^{\prime}\right) \times \overline{\mathcal{M}}\left(B^{\prime}, B^{\prime \prime}\right)$, and this topology coincides with the Hausdorff topology on subsets of $M$ [28].

The next definition shows how to use the above description of the compactified moduli spaces to define a degree and boundary operator satisfying the axioms for abstract topological chains. For this definition we only need to assume that the Morse-Bott function $f: M \rightarrow \mathbb{R}$ is weakly self-indexing (see Lemma 3.6). To simplify the notation we will assume that for each $i=0, \ldots, m$ the components of $B_{i}$, the set of critical points of index $i$, are of the same dimension. In general one needs to group the components by their dimension and then define the degree and boundary operator on each group.

Definition 4.11. Let $B_{i}$ be the set of critical points of index $i$. For any $j=1, \ldots, i$ the degree of $\overline{\mathcal{M}}\left(B_{i}, B_{i-j}\right)$ is defined to be $j+b_{i}-1$ and the boundary operator is defined to be

$$
\partial \overline{\mathcal{M}}\left(B_{i}, B_{i-j}\right)=(-1)^{i+b_{i}} \sum_{i-j<n<i} \overline{\mathcal{M}}\left(B_{i}, B_{n}\right) \times_{B_{n}} \overline{\mathcal{M}}\left(B_{n}, B_{i-j}\right),
$$

where $b_{i}=\operatorname{dim} B_{i}$ and the fibered product is taken over the beginning and endpoint maps $\partial_{-}$and $\partial_{+}$. If $B_{n}=\emptyset$, then $\overline{\mathcal{M}}\left(B_{i}, B_{n}\right)=\overline{\mathcal{M}}\left(B_{n}, B_{i-j}\right)=0$. The boundary operator extends to fibered products of compactified moduli spaces via Definition 4.6 .

Note. For all $p \geq 0$ let $C_{p}$ be the set consisting of the connected components of degree $p$ of fibered products of the form

$$
\overline{\mathcal{M}}\left(B_{i_{1}}, B_{i_{2}}\right) \times_{B_{i_{2}}} \overline{\mathcal{M}}\left(B_{i_{2}}, B_{i_{3}}\right) \times_{B_{i_{3}}} \cdots \times_{B_{i_{n-1}}} \overline{\mathcal{M}}\left(B_{i_{n-1}}, B_{i_{n}}\right),
$$

where $m \geq i_{1}>i_{2}>\cdots>i_{n} \geq 0$ and the fibered products are taken with respect to the beginning and endpoint maps $\partial_{-}$and $\partial_{+}$. Let $S_{p}$ be the free abelian group generated by the elements of $C_{p}$, i.e. $S_{p}=\mathbb{Z}\left[C_{p}\right]$. The preceding definition describes $\overline{\mathcal{M}}\left(B_{i}, B_{i-j}\right)$ and $\partial \overline{\mathcal{M}}\left(B_{i}, B_{i-j}\right)$ as abstract topological chains in $S_{j+b_{i}-1}$ and $S_{j+b_{i}-2}$, respectively. If $\overline{\mathcal{M}}\left(B_{i}, B_{i-j}\right)$ has more than one connected component, then $\overline{\mathcal{M}}\left(B_{i}, B_{i-j}\right) \in S_{j+b_{i}-1}$ is defined to be the sum of these components. Similarly, $\overline{\mathcal{M}}\left(B_{i}, B_{n}\right) \times_{B_{n}} \overline{\mathcal{M}}\left(B_{n}, B_{i-j}\right) \in S_{j+b_{i}-2}$ is defined to be the sum of its connected components.

Notation. We will use the following notation for the fibered product over the beginning and endpoint maps $\partial_{-}$and $\partial_{+}$. For any $i-j<n<i$ we define

$$
\overline{\mathcal{M}}\left(B_{i}, B_{n}, B_{i-j}\right)=\overline{\mathcal{M}}\left(B_{i}, B_{n}\right) \times_{B_{n}} \overline{\mathcal{M}}\left(B_{n}, B_{i-j}\right) .
$$

Similarly for $i-j<n<s<i, \overline{\mathcal{M}}\left(B_{i}, B_{s}, B_{n}, B_{i-j}\right)$ will denote the triple fibered product over $B_{s}$ and $B_{n}$. Iterated fibered products are well defined as abstract 
topological chains since the boundary operator on fibered products is associative by Lemma 4.7 .

Lemma 4.12. The degree and boundary operator for $\overline{\mathcal{M}}\left(B_{i}, B_{i-j}\right)$ satisfy the axioms for abstract topological chains; i.e. the boundary operator on compactified moduli spaces of gradient flow lines is of degree -1 and satisfies $\partial \circ \partial=0$.

Proof. For any $n$ with $i-j<n<i$ the degree of $\overline{\mathcal{M}}\left(B_{i}, B_{n}\right)$ is $i-n+b_{i}-1$ and the degree of $\overline{\mathcal{M}}\left(B_{n}, B_{i-j}\right)$ is $n-i+j+b_{n}-1$ by Definition 4.11. Thus the degree of $\overline{\mathcal{M}}\left(B_{i}, B_{n}\right) \times_{B_{n}} \overline{\mathcal{M}}\left(B_{n}, B_{i-j}\right)$ is $j+b_{i}-2$ by Definition 4.6. We can also apply Definition 4.6] and Definition 4.11 as follows. To simplify the notation let $d=\operatorname{deg} \overline{\mathcal{M}}\left(B_{i}, B_{n}\right)=i-n+b_{i}-1$.

$$
\begin{aligned}
& \partial\left(\overline{\mathcal{M}}\left(B_{i}, B_{n}\right) \times_{B_{n}} \overline{\mathcal{M}}\left(B_{n}, B_{i-j}\right)\right) \\
&= \partial \overline{\mathcal{M}}\left(B_{i}, B_{n}\right) \times_{B_{n}} \overline{\mathcal{M}}\left(B_{n}, B_{i-j}\right)+(-1)^{d+b_{n}} \overline{\mathcal{M}}\left(B_{i}, B_{n}\right) \times_{B_{n}} \partial \overline{\mathcal{M}}\left(B_{n}, B_{i-j}\right) \\
&=(-1)^{i+b_{i}} \sum_{n<s<i} \overline{\mathcal{M}}\left(B_{i}, B_{s}, B_{n}, B_{i-j}\right) \\
&+(-1)^{i+b_{i}-1} \sum_{i-j<t<n} \overline{\mathcal{M}}\left(B_{i}, B_{n}, B_{t}, B_{i-j}\right) .
\end{aligned}
$$

Therefore,

$$
\begin{aligned}
\partial^{2} \overline{\mathcal{M}}\left(B_{i}, B_{i-j}\right)=(-1)^{i+b_{i}}\left[\sum _ { i - j < n < i } \left((-1)^{i+b_{i}} \sum_{n<s<i} \overline{\mathcal{M}}\left(B_{i}, B_{s}, B_{n}, B_{i-j}\right)\right.\right. \\
\left.\left.+(-1)^{i+b_{i}-1} \sum_{i-j<t<n} \overline{\mathcal{M}}\left(B_{i}, B_{n}, B_{t}, B_{i-j}\right)\right)\right] \\
=(-1)^{i+b_{i}}\left[(-1)^{i+b_{i}} \sum_{i-j<n<s<i} \overline{\mathcal{M}}\left(B_{i}, B_{s}, B_{n}, B_{i-j}\right)\right. \\
\left.+(-1)^{i+b_{i}-1} \sum_{i-j<t<n<i} \overline{\mathcal{M}}\left(B_{i}, B_{n}, B_{t}, B_{i-j}\right)\right] \\
=0 .
\end{aligned}
$$

\section{The Morse-Bott-Smale chain complex}

In this section we define the Morse-Bott-Smale chain complex $\left(C_{*}(f), \partial\right)$. Throughout this section we will assume that $f: M \rightarrow \mathbb{R}$ is a Morse-Bott-Smale function on a compact oriented smooth Riemannian manifold $(M, g)$ of dimension $m$. Furthermore, we will assume that all the critical submanifolds $B$ and their negative normal bundles $\nu_{*}^{-}(B)$ are oriented. If $f$ is constant, then the MorseBott-Smale chain complex reduces to the chain complex of smooth singular $N$-cube chains (Example 5.13). If $f$ is a Morse-Smale function, then the Morse-Bott-Smale chain complex reduces to the Morse-Smale-Witten chain complex (Example 5.14). 
5.1. The Morse-Bott degree and the chain complex $\left(\tilde{C}_{*}(f), \partial\right)$. Recall that $B_{i}$ is the set of critical points of index $i$ for $i=0, \ldots, m$ and let $N>\operatorname{dim} M$. For any $p \geq 0$ let $C_{p}$ be the set consisting of the faces of $I^{N}$ of dimension $p$ and the connected components of degree $p$ of fibered products of the form

$$
Q \times_{B_{i_{1}}} \overline{\mathcal{M}}\left(B_{i_{1}}, B_{i_{2}}\right) \times_{B_{i_{2}}} \overline{\mathcal{M}}\left(B_{i_{2}}, B_{i_{3}}\right) \times_{B_{i_{3}}} \cdots \times_{B_{i_{n-1}}} \overline{\mathcal{M}}\left(B_{i_{n-1}}, B_{i_{n}}\right),
$$

where $m \geq i_{1}>i_{2}>\cdots>i_{n} \geq 0, Q$ is a face of $I^{N}$ of dimension $q \leq p, \sigma: Q \rightarrow B_{i_{1}}$ is smooth, and the fibered products are taken with respect to $\sigma$ and the beginning and endpoint maps $\partial_{-}$and $\partial_{+}$. The following lemma is proved at the end of this section.

Lemma 5.1. The elements of $C_{p}$ are compact oriented smooth manifolds with corners.

Let $S_{p}$ be the free abelian group generated by the elements of $C_{p}$, and let $S_{p}^{\infty}\left(B_{i}\right)$ denote the subgroup of the singular $C_{p}$-chain group $S_{p}\left(B_{i}\right)$ generated by those maps $\sigma: P \rightarrow B_{i}$ that satisfy the following two conditions:

(1) The map $\sigma$ is smooth.

(2) If $P \in C_{p}$ is a connected component of a fibered product, then $\sigma=\partial_{+} \circ \pi$, where $\pi$ denotes projection onto the last component of the fibered product.

Definition 5.2. Define the Morse-Bott degree of the singular topological chains in $S_{p}^{\infty}\left(B_{i}\right)$ to be $p+i$. For any $k=0, \ldots, m$ the group of smooth singular topological chains of Morse-Bott degree $k$ is defined to be

$$
\tilde{C}_{k}(f)=\bigoplus_{i=0}^{m} S_{k-i}^{\infty}\left(B_{i}\right) .
$$

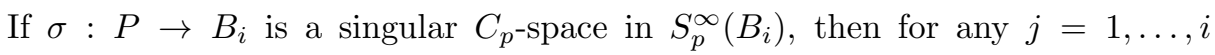
composing the projection map $\pi_{2}$ onto the second component of $P \times{ }_{B_{i}} \overline{\mathcal{M}}\left(B_{i}, B_{i-j}\right)$ with the endpoint map $\partial_{+}: \overline{\mathcal{M}}\left(B_{i}, B_{i-j}\right) \rightarrow B_{i-j}$ gives a map

$$
P \times_{B_{i}} \overline{\mathcal{M}}\left(B_{i}, B_{i-j}\right) \stackrel{\pi_{2}}{\longrightarrow} \overline{\mathcal{M}}\left(B_{i}, B_{i-j}\right) \stackrel{\partial_{+}}{\longrightarrow} B_{i-j} .
$$

The next lemma shows that restricting this map to the connected components of the fibered product $P \times_{B_{i}} \overline{\mathcal{M}}\left(B_{i}, B_{i-j}\right)$ and adding these restrictions (with the sign determined by the orientation when the dimension of a component is zero) defines an element $\partial_{j}(\sigma) \in S_{p+j-1}^{\infty}\left(B_{i-j}\right)$.

Lemma 5.3. If $\sigma: P \rightarrow B_{i}$ is a singular $C_{p}$-space in $S_{p}^{\infty}\left(B_{i}\right)$, then for any $j=1, \ldots, i$ adding the components of $P \times_{B_{i}} \overline{\mathcal{M}}\left(B_{i}, B_{i-j}\right)$ (with sign when the dimension of a component is zero) yields an abstract topological chain of degree $p+j-1$. That is, we can identify

$$
P \times_{B_{i}} \overline{\mathcal{M}}\left(B_{i}, B_{i-j}\right) \in S_{p+j-1} .
$$

Thus, for all $j=1, \ldots, i$ there is an induced homomorphism

$$
\partial_{j}: S_{p}^{\infty}\left(B_{i}\right) \rightarrow S_{p+j-1}^{\infty}\left(B_{i-j}\right)
$$

which decreases the Morse-Bott degree by 1.

Proof. The set $B_{i}$ is a union of submanifolds of $M$, possibly of different dimensions. Since $\sigma: P \rightarrow B_{i}$ is continuous and $P$ is connected, the image $\sigma(P)$ must lie in a connected component $B \subseteq B_{i}$. By Lemma 4.12, $\overline{\mathcal{M}}\left(B, B_{i-j}\right)$ is an abstract 
topological chain of degree $j+b-1$, and by the discussion after Lemma 4.5, $P \times_{B}$ $\overline{\mathcal{M}}\left(B, B_{i-j}\right)$ has degree $p+j-1$. This degree is independent of the dimension of the connected component $B$.

To see that $P \times_{B_{i}} \overline{\mathcal{M}}\left(B_{i}, B_{i-j}\right)$ has finitely many components, first note that $P \in C_{p}$ is a compact smooth manifold with corners by Lemma 5.1, and if $B^{\prime}$ is a connected component of $B_{i-j}$, then $\overline{\mathcal{M}}\left(B, B^{\prime}\right)$ is a compact smooth manifold with corners by Theorem 4.9. We will show in Lemma 5.21 that

$$
P \times{ }_{B} \overline{\mathcal{M}}\left(B, B^{\prime}\right)
$$

is a compact smooth manifold with corners, and hence it has finitely many components. By assumption (see Definition 3.1), the set $B_{i-j}$ has finitely many components. Thus,

$$
P \times_{B} \overline{\mathcal{M}}\left(B, B_{i-j}\right)=P \times_{B_{i}} \overline{\mathcal{M}}\left(B_{i}, B_{i-j}\right)
$$

has finitely many components.

Definition 5.4. For $k=1, \ldots, m$ define a homomorphism $\partial: \tilde{C}_{k}(f) \rightarrow \tilde{C}_{k-1}(f)$ as follows. If $\sigma \in S_{p}^{\infty}\left(B_{i}\right)$ is a singular $S_{p}$-space of $B_{i}$ where $p=k-i$, then

$$
\partial(\sigma)=\bigoplus_{j=0}^{m} \partial_{j}(\sigma)
$$

where $\partial_{0}$ is $(-1)^{k}$ times the boundary operator on singular topological chains defined in Section 4, $\partial_{j}(\sigma)=\partial_{+} \circ \pi_{2}: P \times_{B_{i}} \overline{\mathcal{M}}\left(B_{i}, B_{i-j}\right) \rightarrow B_{i-j}$ for $j=1, \ldots, i$, and $\partial_{j}(\sigma)=0$ otherwise. The map $\partial$ extends to a homomorphism

$$
\partial: \bigoplus_{i=0}^{m} S_{k-i}^{\infty}\left(B_{i}\right) \longrightarrow \bigoplus_{i=0}^{m} S_{k-1-i}^{\infty}\left(B_{i}\right) .
$$

When $m=2$ this homomorphism can be pictured as follows:

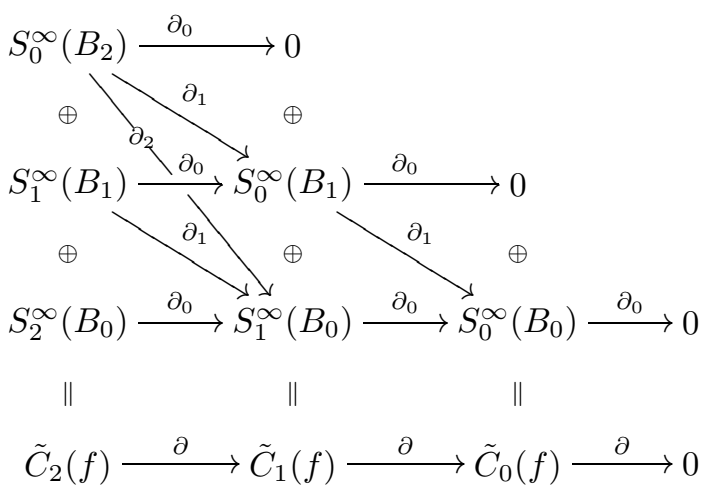

Proposition 5.5. For every $j=0, \ldots, m$

$$
\sum_{q=0}^{j} \partial_{q} \partial_{j-q}=0 .
$$

Proof. The case $j=0$ follows from Lemma 4.7 and Lemma 4.12 Now, let $\sigma \in$ $S_{p}^{\infty}\left(B_{i}\right)$ be a singular $C_{p}$-space of $B_{i}$. Since $\partial_{q}\left(\partial_{j-q}(\sigma)\right)=0$ if $j>i$, we will assume that $1 \leq j \leq i$. To simplify the notation we give the following computation in terms of abstract topological chains. This is sufficient because in $\tilde{C}_{*}(f)$ the only 
map allowed from these fibered products is the endpoint map $\partial_{+}$composed with the projection map onto the last component. We will also assume that for every $i=0, \ldots, m$ all the components of $B_{i}$ are of the same dimension $b_{i}$; in general, the components need to be grouped by their dimension and the computation repeated on each group.

When $q=0$ we use Definitions 4.6, 4.11, and 5.4 to compute as follows:

$$
\begin{aligned}
\partial_{0}\left(\partial_{j}(P)\right)= & \partial_{0}\left(P \times_{B_{i}} \overline{\mathcal{M}}\left(B_{i}, B_{i-j}\right)\right) \\
= & (-1)^{p+i-1}\left(\partial P \times_{B_{i}} \overline{\mathcal{M}}\left(B_{i}, B_{i-j}\right)+(-1)^{p+b_{i}} P \times_{B_{i}} \partial \overline{\mathcal{M}}\left(B_{i}, B_{i-j}\right)\right) \\
= & (-1)^{p+i-1} \partial P \times_{B_{i}} \overline{\mathcal{M}}\left(B_{i}, B_{i-j}\right) \\
& \quad+(-1)^{2 p+2 b_{i}+2 i-1} \sum_{i-j<n<i} P \times_{B_{i}} \overline{\mathcal{M}}\left(B_{i}, B_{n}\right) \times_{B_{n}} \overline{\mathcal{M}}\left(B_{n}, B_{i-j}\right) .
\end{aligned}
$$

If $1 \leq q \leq j-1$, then by Definition 5.4

$$
\partial_{q}\left(\partial_{j-q}(P)\right)=P \times_{B_{i}} \overline{\mathcal{M}}\left(B_{i}, B_{i-j+q}\right) \times_{B_{i-j+q}} \overline{\mathcal{M}}\left(B_{i-j+q}, B_{i-j}\right),
$$

and if $q=j$, then

$$
\partial_{j}\left(\partial_{0}(P)\right)=(-1)^{p+i} \partial P \times_{B_{i}} \overline{\mathcal{M}}\left(B_{i}, B_{i-j}\right) .
$$

Summing these expressions gives the desired result.

Corollary 5.6. The pair $\left(\tilde{C}_{*}(f), \partial\right)$ is a chain complex, i.e. $\partial \circ \partial=0$.

5.2. Orientations. In this subsection we describe an explicit set of orientations on the elements of $C_{p}$.

Recall the assumption that every critical submanifold $B$ and every negative normal bundle $\nu_{*}^{-}(B)$ are oriented. For any $p \in B$, the relation

$$
T_{p} M=T_{p} B \oplus \nu_{p}^{+}(B) \oplus \nu_{p}^{-}(B)
$$

determines an orientation on $\nu_{p}^{+}(B)$. The stable and unstable manifolds are oriented by requiring that the injective immersions $E^{+}: \nu_{*}^{+}(B) \rightarrow W^{s}(B)$ and $E^{-}: \nu_{*}^{-}(B) \rightarrow W^{u}(B)$ are orientation preserving (see Theorem 3.3). If $N \subseteq M$ is an oriented submanifold, then the normal bundle of $N$ is oriented by the relation $T_{x}(N) \oplus \nu_{x}(N)=T_{x}(M)$ for all $x \in N$. For any two connected critical submanifolds $B$ and $B^{\prime}$, the orientation on $W\left(B, B^{\prime}\right)=W^{u}(B) \pitchfork W^{s}\left(B^{\prime}\right)$ is determined by the relation

$$
T_{x}(M)=T_{x} W\left(B, B^{\prime}\right) \oplus \nu_{x}\left(W^{s}\left(B^{\prime}\right)\right) \oplus \nu_{x}\left(W^{u}(B)\right)
$$

for all $x \in W\left(B, B^{\prime}\right)$. Picking a non-critical value $a$ between $f\left(B^{\prime}\right)$ and $f(B)$, we can identify $\mathcal{M}\left(B, B^{\prime}\right)=f^{-1}(a) \cap W\left(B, B^{\prime}\right)$. An orientation on $\mathcal{M}\left(B, B^{\prime}\right)$ is then determined by

$$
T_{x} W\left(B, B^{\prime}\right)=\operatorname{span}((-\nabla f)(x)) \oplus T_{x} \mathcal{M}\left(B, B^{\prime}\right)
$$

for all $x \in f^{-1}(a) \cap W\left(B, B^{\prime}\right)$. This determines an orientation on the compact manifold with boundary $\overline{\mathcal{M}}\left(B, B^{\prime}\right)$ (see for instance Section VI.9 of [14]).

Definition 5.7. Suppose that $B$ is an oriented smooth manifold without boundary and $P_{1}$ and $P_{2}$ are oriented smooth manifolds with corners. If $\sigma_{1}: P_{1} \rightarrow B$ and $\sigma_{2}$ : $P_{2} \rightarrow B$ are smooth maps that intersect transversally and stratum transversally, then the orientation on the smooth manifold with corners $P_{1} \times_{B} P_{2}$ is defined by 
the relation

$$
(-1)^{(\operatorname{dim} B)\left(\operatorname{dim} P_{2}\right)} T_{*}\left(P_{1} \times{ }_{B} P_{2}\right) \oplus\left(\sigma_{1} \times \sigma_{2}\right)^{*}\left(\nu_{*}(\Delta(B))\right)=T_{*}\left(P_{1} \times P_{2}\right),
$$

where $\nu_{*}(\Delta(B))$ denotes the normal bundle of the diagonal in $B \times \mathrm{B}$.

Note. Lemma 4.5 extends to the category of smooth manifolds with corners under some additional assumptions; see the end of this section for more details. Also, the normal bundle of the diagonal $\Delta(B) \subset B \times B$ pulls back to the normal bundle of the fibered product $P_{1} \times_{B} P_{2}$ in $P_{1} \times P_{2}$ via $\sigma_{1} \times \sigma_{2}: P_{1} \times P_{2} \rightarrow B \times B$.

Lemma 5.8. The above orientation on fibered products of transverse intersections of smooth manifolds with corners is associative, i.e.

$$
\left(P_{1} \times_{B_{1}} P_{2}\right) \times_{B_{2}} P_{3}=P_{1} \times_{B_{1}}\left(P_{2} \times_{B_{2}} P_{3}\right)
$$

as oriented smooth manifolds with corners.

Proof. The proof is a straightforward computation. The $\operatorname{sign}(-1)^{(\operatorname{dim} B)\left(\operatorname{dim} P_{2}\right)}$ is needed to prove associativity.

We will see in the proof of Lemma 5.1 that Definition 5.7 applies to the fibered products in $C_{p}$, and hence by Lemma 5.8 there is a well-defined orientation on the components of

$$
Q \times_{B_{i_{1}}} \overline{\mathcal{M}}\left(B_{i_{1}}, B_{i_{2}}\right) \times_{B_{i_{2}}} \overline{\mathcal{M}}\left(B_{i_{2}}, B_{i_{3}}\right) \times_{B_{i_{3}}} \cdots \times_{B_{i_{n-1}}} \overline{\mathcal{M}}\left(B_{i_{n-1}}, B_{i_{n}}\right)
$$

in $C_{p}$.

Note. The boundary of $\overline{\mathcal{M}}\left(B, B^{\prime}\right)$ consists of fibered products of compactified moduli spaces of gradient flow lines by Theorem 4.8 , These fibered products are oriented by Definition 5.7 The boundary of $\overline{\mathcal{M}}\left(B, B^{\prime}\right)$ also inherits an orientation from the orientation on $\overline{\mathcal{M}}\left(B, B^{\prime}\right)$ (see for instance Lemma VI.9.1 of [14]). These two orientations can be compared by using Proposition 2.7 of [4, which says that the gluing map is orientation reversing. We will always use the orientation given by Definition 5.7 .

5.3. Degenerate and non-degenerate singular topological chains. The Morse-Bott-Smale chain complex $\left(C_{*}(f), \partial\right)$ is defined as the quotient of the chain complex $\left(\tilde{C}_{*}(f), \partial\right)$ by the degenerate singular topological chains.

Definition 5.9 (Degeneracy relations for the Morse-Bott-Smale chain complex). Let $\sigma_{P}, \sigma_{Q} \in S_{p}^{\infty}\left(B_{i}\right)$ be singular $C_{p}$-spaces in $B_{i}$ and let $\partial Q=\sum_{j} n_{j} Q_{j} \in S_{p-1}$. For any map

$$
\alpha: P \rightarrow Q
$$

let $\partial_{0} \sigma_{Q} \circ \alpha$ denote the formal sum $\left.(-1)^{p+i} \sum_{j} n_{j}\left(\sigma_{Q} \circ \alpha\right)\right|_{\alpha^{-1}\left(Q_{j}\right)}$. Define the subgroup $D_{p}^{\infty}\left(B_{i}\right) \subseteq S_{p}^{\infty}\left(B_{i}\right)$ of degenerate singular topological chains to be the subgroup generated by the following elements:

(1) If $\alpha$ is an orientation preserving homeomorphism such that $\sigma_{Q} \circ \alpha=\sigma_{P}$ and $\partial_{0} \sigma_{Q} \circ \alpha=\partial_{0} \sigma_{P}$, then $\sigma_{P}-\sigma_{Q} \in D_{p}^{\infty}\left(B_{i}\right)$.

(2) If $P$ is a face of $I^{N}$ and $\sigma_{P}$ does not depend on some free coordinate of $P$, then $\sigma_{P} \in D_{p}^{\infty}\left(B_{i}\right)$ and $\partial_{j}\left(\sigma_{P}\right) \in D_{p+j-1}^{\infty}\left(B_{i-j}\right)$ for all $j=1, \ldots, m$.

(3) If $P$ and $Q$ are connected components of some fibered products and $\alpha$ is an orientation reversing map such that $\sigma_{Q} \circ \alpha=\sigma_{P}$ and $\partial_{0} \sigma_{Q} \circ \alpha=\partial_{0} \sigma_{P}$, then $\sigma_{P}+\sigma_{Q} \in D_{p}^{\infty}\left(B_{i}\right)$. 
(4) If $Q$ is a face of $I^{N}$ and $R$ is a connected component of a fibered product $Q \times_{B_{i_{1}}} \overline{\mathcal{M}}\left(B_{i_{1}}, B_{i_{2}}\right) \times_{B_{i_{2}}} \overline{\mathcal{M}}\left(B_{i_{2}}, B_{i_{3}}\right) \times_{B_{i_{3}}} \cdots \times_{B_{i_{n-1}}} \overline{\mathcal{M}}\left(B_{i_{n-1}}, B_{i_{n}}\right)$ such that $\operatorname{deg} R>\operatorname{dim} B_{i_{n}}$, then $\sigma_{R} \in D_{r}^{\infty}\left(B_{i_{n}}\right)$ and $\partial_{j}\left(\sigma_{R}\right) \in D_{r+j-1}^{\infty}\left(B_{i_{n}-j}\right)$ for all $j=0, \ldots, m$.

(5) If $\sum_{\alpha} n_{\alpha} \sigma_{\alpha} \in S_{*}(R)$ is a smooth singular chain in a connected component $R$ of a fibered product (as in (4)) that represents the fundamental class of $R$ and

$$
(-1)^{r+i_{n}} \partial_{0} \sigma_{R}-\sum_{\alpha} n_{\alpha} \partial\left(\sigma_{R} \circ \sigma_{\alpha}\right)
$$

is in the group generated by the elements satisfying one of the above conditions, then

$$
\sigma_{R}-\sum_{\alpha} n_{\alpha}\left(\sigma_{R} \circ \sigma_{\alpha}\right) \in D_{r}^{\infty}\left(B_{i_{n}}\right)
$$

and

$$
\partial_{j}\left(\sigma_{R}-\sum_{\alpha} n_{\alpha}\left(\sigma_{R} \circ \sigma_{\alpha}\right)\right) \in D_{r+j-1}^{\infty}\left(B_{i_{n}-j}\right)
$$

for all $j=1, \ldots, m$.

Note. Condition (3) does not apply to the $p$-faces of $I^{N}$. If we allowed condition (3) to apply to the $p$-faces of $I^{N}$, then we could combine conditions (1) and (3). However, the resulting chain complex would then reduce to a quotient of the chain complex of smooth singular cubes when $f: M \rightarrow \mathbb{R}$ is a constant function (see Example 5.13).

Lemma 5.10. For any $i, j=0, \ldots, m$ there is an induced homomorphism

$$
\partial_{j}: S_{p}^{\infty}\left(B_{i}\right) / D_{p}^{\infty}\left(B_{i}\right) \rightarrow S_{p+j-1}^{\infty}\left(B_{i-j}\right) / D_{p+j-1}^{\infty}\left(B_{i-j}\right) .
$$

Proof. Let $\sigma_{P}: P \rightarrow B_{i}$ be a singular $C_{p}$-space in $S_{p}^{\infty}\left(B_{i}\right)$ such that $\sigma_{Q} \circ \alpha=\sigma_{P}$ and $\partial_{0} \sigma_{Q} \circ \alpha=\partial_{0} \sigma_{P}$ for some singular $C_{p}$-space $\sigma_{Q}: Q \rightarrow B_{i}$ in $S_{p}^{\infty}\left(B_{i}\right)$ and some orientation preserving homeomorphism $\alpha: P \rightarrow Q$. By Proposition 5.5. $\partial_{0}^{2} \sigma_{Q}=\partial_{0}^{2} \sigma_{P}=0$, and hence $\partial_{0}\left(\partial_{0} \sigma_{Q}\right) \circ \alpha=\partial_{0}\left(\partial_{0} \sigma_{P}\right)$. Thus, $\partial_{0}\left(\sigma_{P}-\sigma_{Q}\right)=$ $\partial_{0} \sigma_{P}-\partial_{0} \sigma_{Q} \in D_{p-1}^{\infty}\left(B_{i}\right)$. Now assume that $1 \leq j \leq i$ and recall that $\partial_{j}\left(\sigma_{P}\right)$ is the map

$$
\partial_{j}\left(\sigma_{P}\right): P \times_{B_{i}} \overline{\mathcal{M}}\left(B_{i}, B_{i-j}\right) \stackrel{\pi_{2}}{\longrightarrow} \overline{\mathcal{M}}\left(B_{i}, B_{i-j}\right) \stackrel{\partial_{+}}{\longrightarrow} B_{i-j}
$$

where the fibered product is taken over $\sigma_{P}: P \rightarrow B_{i}$ and $\partial_{-}: \overline{\mathcal{M}}\left(B_{i}, B_{i-j}\right) \rightarrow B_{i}$. The map $\beta: P \times_{B_{i}} \overline{\mathcal{M}}\left(B_{i}, B_{i-j}\right) \rightarrow Q \times_{B_{i}} \overline{\mathcal{M}}\left(B_{i}, B_{i-j}\right)$ defined by $\beta(p, \gamma)=$ $(\alpha(p), \gamma)$ is an orientation preserving homeomorphism that satisfies $\partial_{j}\left(\sigma_{Q}\right) \circ \beta=$ $\partial_{j}\left(\sigma_{P}\right)$ since $\sigma_{Q} \circ \alpha=\sigma_{P}$. By Definition 4.6.

$$
\partial\left(P \times_{B_{i}} \overline{\mathcal{M}}\left(B_{i}, B_{i-j}\right)\right)=\partial P \times_{B_{i}} \overline{\mathcal{M}}\left(B_{i}, B_{i-j}\right)+(-1)^{p+b_{i}} P \times_{B_{i}} \partial \overline{\mathcal{M}}\left(B_{i}, B_{i-j}\right)
$$

and

$$
\partial\left(Q \times_{B_{i}} \overline{\mathcal{M}}\left(B_{i}, B_{i-j}\right)\right)=\partial Q \times_{B_{i}} \overline{\mathcal{M}}\left(B_{i}, B_{i-j}\right)+(-1)^{p+b_{i}} Q \times_{B_{i}} \partial \overline{\mathcal{M}}\left(B_{i}, B_{i-j}\right) .
$$

The assumption $\partial_{0} \sigma_{Q} \circ \alpha=\partial_{0} \sigma_{P}$ implies that $\partial_{0}\left(\partial_{j}\left(\sigma_{Q}\right)\right) \circ \beta=\partial_{0}\left(\partial_{j}\left(\sigma_{P}\right)\right)$, and thus $\partial_{j}\left(\sigma_{P}-\sigma_{Q}\right)=\partial_{j} \sigma_{P}-\partial_{j} \sigma_{Q} \in D_{p+j-1}^{\infty}\left(B_{i-j}\right)$ by the first condition for degeneracy. This completes the proof for the first case. The proof for the third case is similar.

Now assume that $\sigma_{P}: P \rightarrow B_{i}$ is a singular $C_{p}$-space in $S_{p}^{\infty}\left(B_{i}\right)$, where $P$ is a face of $I^{N}$ and $\sigma_{P}$ does not depend on some free coordinate $x_{j}$. Then in the sum for 
$\partial_{0} \sigma_{P}$ the term $\left.\sigma_{P}\right|_{x_{j}=0}-\left.\sigma_{P}\right|_{x_{j}=1} \in D_{p-1}^{\infty}\left(B_{i}\right)$ because it satisfies the first condition for degeneracy, and the rest of the terms are all independent of the coordinate corresponding to $x_{j}$ and thus satisfy the second condition for degeneracy. Thus, $\partial_{0} \sigma_{P} \in D_{p-1}^{\infty}\left(B_{i}\right)$. If $j>0$, then the second condition for degeneracy implies that $\partial_{j}\left(\sigma_{P}\right) \in D_{p+j-1}^{\infty}\left(B_{i-j}\right)$. This completes the proof for the second case of Definition 5.9. The fourth and fifth cases follow immediately from the definition.

Definition 5.11. Define

$$
C_{p}\left(B_{i}\right)=S_{p}^{\infty}\left(B_{i}\right) / D_{p}^{\infty}\left(B_{i}\right)
$$

to be the group of non-degenerate smooth singular topological chains in $S_{p}^{\infty}\left(B_{i}\right)$. The group $C_{k}(f)$ of $k$-chains in the Morse-Bott chain complex of $f$ is defined to be the group of non-degenerate smooth singular topological chains of Morse-Bott degree $k$, i.e.

$$
C_{k}(f)=\bigoplus_{i=0}^{m} C_{k-i}\left(B_{i}\right)=\bigoplus_{i=0}^{m} S_{k-i}^{\infty}\left(B_{i}\right) / D_{k-i}^{\infty}\left(B_{i}\right) .
$$

The boundary operator in the Morse-Bott-Smale chain complex

$$
\partial: \bigoplus_{i=0}^{m} S_{k-i}^{\infty}\left(B_{i}\right) / D_{k-i}^{\infty}\left(B_{i}\right) \longrightarrow \bigoplus_{i=0}^{m} S_{k-1-i}^{\infty}\left(B_{i}\right) / D_{k-1-i}^{\infty}\left(B_{i}\right)
$$

is defined to be $\partial=\bigoplus_{j=0}^{m} \partial_{j}$, where $\partial_{j}$ is the induced homomorphism from Lemma 5.10 .

When $m=2$ this homomorphism can be pictured as follows:

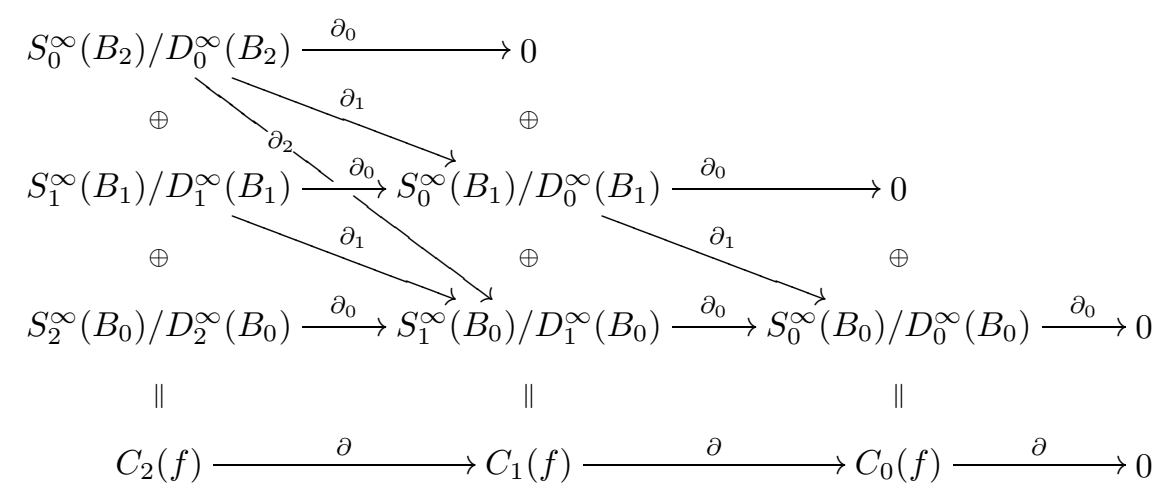

Corollary 5.12. The pair $\left(C_{*}(f), \partial\right)$ is a chain complex, i.e. $\partial \circ \partial=0$.

Proof. This follows from Proposition 5.5 and Lemma 5.10.

\subsection{Computing Morse-Bott homology.}

Example 5.13 (A constant function).

Let $f: M \rightarrow \mathbb{R}$ be a constant function on a compact oriented smooth Riemannian manifold $(M, g)$, and let $N>\operatorname{dim} M$. Since $f$ is constant there is only one critical submanifold $B_{0}=M$, and for any $p \geq 0$ the set $C_{p}$ consists of the faces of $I^{N}$ of dimension $p$. For any $k \geq 0$ the group $\tilde{C}_{k}(f)=S_{k}^{\infty}\left(B_{0}\right)$ is the subgroup of smooth chains in the group $S_{k}(B)$ of continuous singular $N$-cube chains of degree $k, \partial_{0}=(-1)^{k} \partial$, and $\partial_{j}$ is trivial for all $j>0$. 
Conditions (1) and (2) for degeneracy in Definition 5.9 agree with the two conditions for degeneracy in Definition 4.3, and the other conditions are vacuous since $B_{i}=\emptyset$ for all $i>0$. Thus, $D_{k}^{\infty}\left(B_{0}\right)$ is the subgroup smooth chains in the group $D_{k}\left(B_{0}\right)$ of continuous degenerate singular $N$-cube chains, and the Morse-BottSmale chain complex $\left(C_{*}(f), \partial\right)$, with its boundary operator multiplied by $(-1)^{k}$, is the subchain complex of the chain complex $\left(S_{*}(B) / D_{*}(B), \partial_{*}\right)$ from Theorem 4.4 consisting of non-degenerate smooth singular $N$-cube chains. The proof of Theorem 4.4 carries over verbatim to the smooth case, and standard arguments can be used to show that the homology of the smooth singular cube chain complex of a smooth manifold $M$ is isomorphic to the homology of the continuous singular cube chain complex; cf. Chapter 5 of 43 . Thus, the homology of the Morse-Bott-Smale chain complex $\left(C_{*}(f), \partial\right)$ is isomorphic to the singular homology $H_{*}(M ; \mathbb{Z})$. When $m=2$, this chain complex can be pictured as follows:

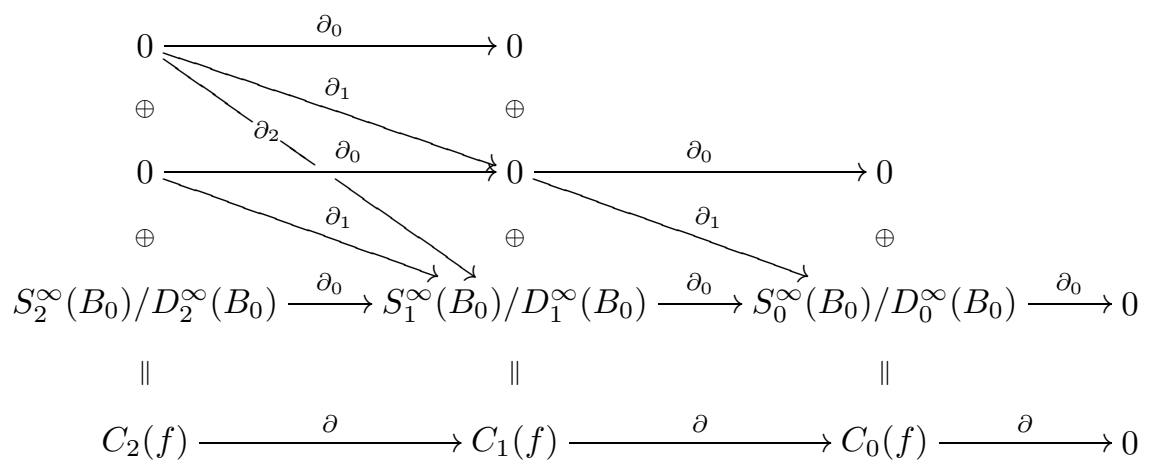

Example 5.14 (A Morse-Smale function).

Let $f: M \rightarrow \mathbb{R}$ be a Morse-Smale function on a compact oriented smooth Riemannian manifold $(M, g)$, and let $N>\operatorname{dim} M$. Since the critical points of $f$ are isolated, $\operatorname{dim} B_{i}=0$ for all $i$ and conditions (2) and (4) for degeneracy in Definition 5.9 imply that $D_{p}^{\infty}\left(B_{i}\right)=S_{p}^{\infty}\left(B_{i}\right)$ for all $i \geq 0$ and $p>0$. This implies that $\partial_{j}$ is trivial unless $j=1$ (see Lemma 5.3).

Moreover, the first condition for degeneracy in Definition 5.9 implies that $C_{k}(f)=$ $S_{0}\left(B_{k}\right) / D_{0}\left(B_{k}\right)$ is isomorphic to the free abelian group generated by the critical points of index $k$ for all $k=0, \ldots, m$. If $q \in B_{k}$ is a critical point of index $k$, then the map $\partial_{1}$ applied to $q$,

$$
\partial_{1}(q): q \times_{B_{k}} \overline{\mathcal{M}}\left(B_{k}, B_{k-1}\right) \stackrel{\pi_{2}}{\longrightarrow} \overline{\mathcal{M}}\left(B_{k}, B_{k-1}\right) \stackrel{\partial_{+}}{\longrightarrow} B_{k-1},
$$

counts the number of gradient flow lines (with sign) between $q$ and the critical points in $B_{k-1}$, i.e.

$$
\partial_{1}(q)=\sum_{p \in B_{k-1}} n(q, p) p \in C_{k-1}(f),
$$

where $n(q, p)$ is the number of gradient flow lines between $q$ and $p$ counted with the signs coming from the orientation of the zero-dimensional manifold $\overline{\mathcal{M}}\left(B_{k}, B_{k-1}\right)$. Hence, $\left(C_{*}(f), \partial\right)$ is the Morse-Smale-Witten chain complex, and the homology of $\left(C_{*}(f), \partial\right)$ is isomorphic to the singular homology $H_{*}(M ; \mathbb{Z})$ by the Morse Homology 
Theorem. When $m=2$, this chain complex can be pictured as follows:

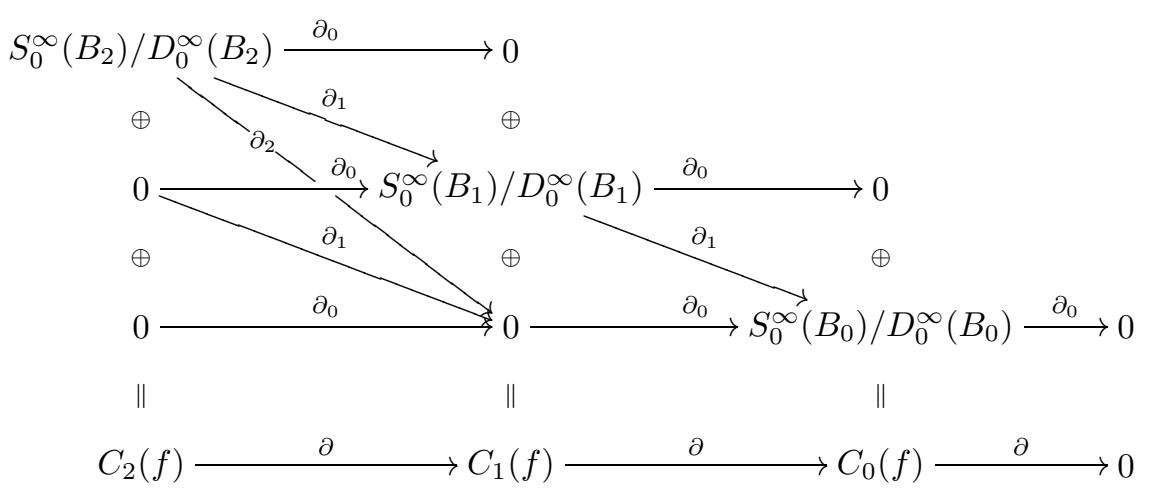

Example 5.15 (A Morse-Bott-Smale function on $S^{2}$ ).

Consider $M=S^{2}=\left\{(x, y, z) \in \mathbb{R}^{3} \mid x^{2}+y^{2}+z^{2}=1\right\}$, and let $f(x, y, z)=z^{2}$. Then $B_{0} \approx S^{1}, B_{1}=\emptyset$, and $B_{2}=\{n, s\}$ where $n=(0,0,1)$ and $s=(0,0,-1)$.

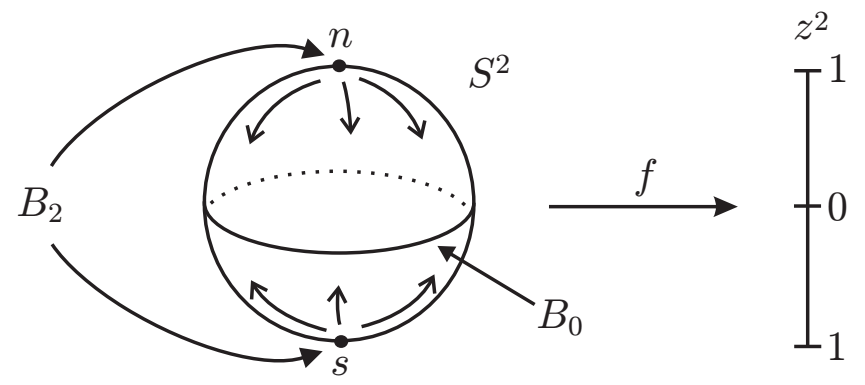

Note that

$$
S_{0}^{\infty}\left(B_{2}\right) / D_{0}^{\infty}\left(B_{2}\right) \approx\langle n, s\rangle \approx \mathbb{Z} \oplus \mathbb{Z}
$$

by the first two conditions for degeneracy, and $S_{p}^{\infty}\left(B_{2}\right) / D_{p}^{\infty}\left(B_{2}\right)=0$ for $p>0$ by the second condition for degeneracy in Definition 5.9. Thus, the Morse-Bott-Smale chain complex can be pictured as follows:

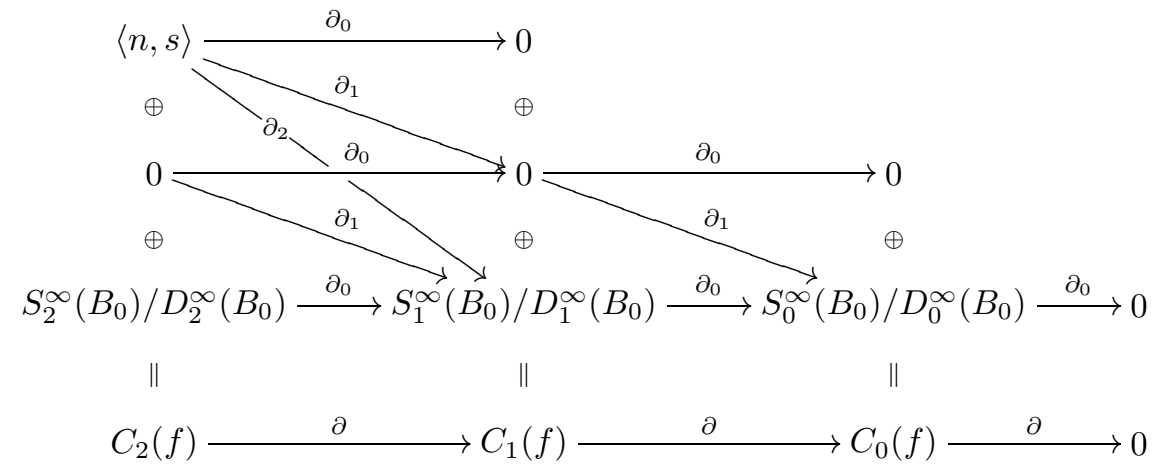

The group $S_{k}^{\infty}\left(B_{0}\right) / D_{k}^{\infty}\left(B_{0}\right)$ is non-trivial for all $k \leq N$, but

$$
H_{k}\left(C_{*}(f), \partial\right)=0 \quad \text { if } \quad k>2
$$


and $\partial_{0}: S_{3}^{\infty}\left(B_{0}\right) / D_{3}^{\infty}\left(B_{0}\right) \rightarrow S_{2}^{\infty}\left(B_{0}\right) / D_{2}^{\infty}\left(B_{0}\right)$ maps onto the kernel of the boundary operator $\partial_{0}: S_{2}^{\infty}\left(B_{0}\right) / D_{2}^{\infty}\left(B_{0}\right) \rightarrow S_{1}^{\infty}\left(B_{0}\right) / D_{1}^{\infty}\left(B_{0}\right)$ because the homology of the bottom row in the above diagram is the smooth integral singular homology of $B_{0} \approx S^{1}$.

The moduli space $\overline{\mathcal{M}}\left(B_{2}, B_{0}\right)$ is a disjoint union of two copies of $S^{1}$ with opposite orientations. This moduli space can be viewed as a subset of the manifold $S^{2}$ since $\overline{\mathcal{M}}\left(B_{2}, B_{0}\right)=\mathcal{M}\left(B_{2}, B_{0}\right)$.

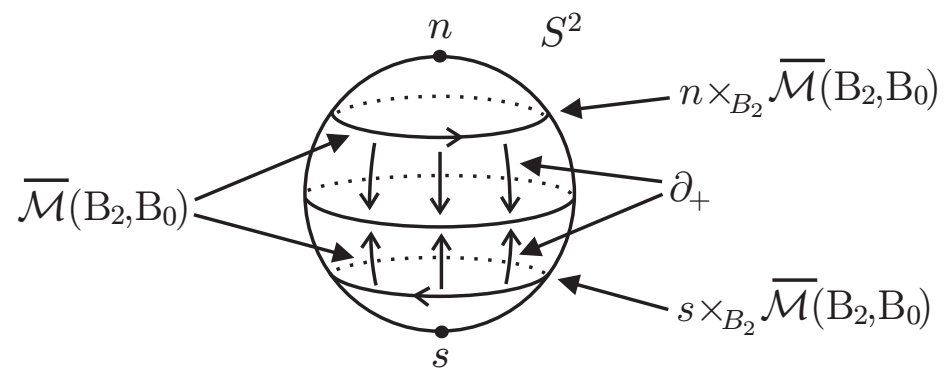

Thus, there is an orientation reversing map $\alpha: n \times{ }_{n} \overline{\mathcal{M}}\left(B_{2}, B_{0}\right) \rightarrow s \times{ }_{s} \overline{\mathcal{M}}\left(B_{2}, B_{0}\right)$ such that $\partial_{2}(n) \circ \alpha=\partial_{2}(s)$. Since $\partial_{0}\left(\partial_{2}(n)\right)=\partial_{0}\left(\partial_{2}(s)\right)=0$, the third condition for degeneracy from Definition 5.9 implies that

$$
\partial_{2}(n+s)=\partial_{2}(n)+\partial_{2}(s)=0 \in S_{1}\left(B_{0}\right) / D_{1}\left(B_{0}\right),
$$

and the fifth condition for degeneracy implies that $\partial_{2}$ maps either $n$ or $s$ onto a representative of the generator of

$$
\frac{\operatorname{ker} \partial_{0}: S_{1}^{\infty}\left(B_{0}\right) / D_{1}^{\infty}\left(B_{0}\right) \rightarrow S_{0}^{\infty}\left(B_{0}\right) / D_{0}^{\infty}\left(B_{0}\right)}{\operatorname{im} \partial_{0}: S_{2}^{\infty}\left(B_{0}\right) / D_{2}^{\infty}\left(B_{0}\right) \rightarrow S_{1}^{\infty}\left(B_{0}\right) / D_{1}^{\infty}\left(B_{0}\right)} \approx H_{1}\left(S^{1} ; \mathbb{Z}\right) \approx \mathbb{Z}
$$

depending on the orientation chosen for $B_{0}$. Therefore,

$$
H_{k}\left(C_{*}(f), \partial\right)= \begin{cases}\mathbb{Z} & \text { if } k=0,2, \\ 0 & \text { otherwise. }\end{cases}
$$

Example 5.16 (Another Morse-Bott-Smale function on $S^{2}$ ).

Consider $M=S^{2}=\left\{(x, y, z) \in \mathbb{R}^{3} \mid x^{2}+y^{2}+z^{2}=1\right\}$, and let $f(x, y, z)=-z^{2}$. Then $B_{0}=\{n, s\}$, where $n=(0,0,1)$ and $s=(0,0,-1), B_{1} \approx S^{1}$, and $B_{2}=\emptyset$.

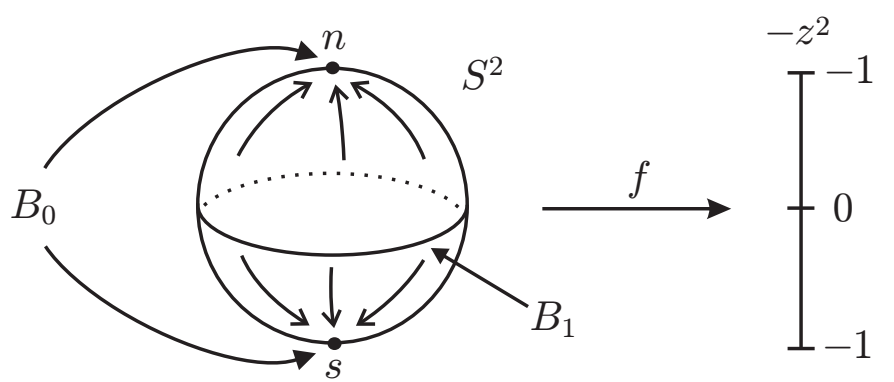

We have

$$
S_{0}^{\infty}\left(B_{0}\right) / D_{0}^{\infty}\left(B_{0}\right) \approx\langle n, s\rangle \approx \mathbb{Z} \oplus \mathbb{Z}
$$


by the first two conditions for degeneracy, and $S_{p}^{\infty}\left(B_{0}\right) / D_{p}^{\infty}\left(B_{0}\right)=0$ for $p>0$ by the second and fourth conditions for degeneracy in Definition 5.9. Thus, the Morse-Bott-Smale chain complex can be pictured as follows:

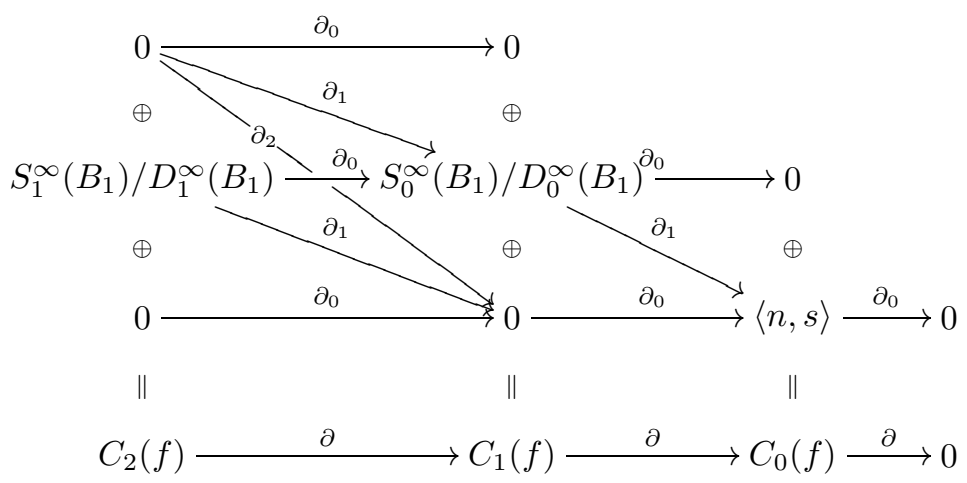

The middle row computes the smooth integral singular homology of $B_{1} \approx S^{1}$ when $k>1$, and hence $H_{2}\left(C_{*}(f), \partial\right)=\mathbb{Z}$. However, for $k=1$ the kernel of $\partial=\partial_{0} \oplus \partial_{1}$ : $C_{1}(f) \rightarrow C_{0}(f)$ is trivial because $\partial_{1}\left(\sigma_{P}\right)$ is non-zero for any map $\sigma_{P}: P \rightarrow B_{1}$ from a point $P \in C_{0}$ to $B_{1}$. In fact, the fibered product $P \times_{B_{1}} \overline{\mathcal{M}}\left(B_{1}, B_{0}\right)$ consists of two points, and

$$
\partial_{1}\left(\sigma_{P}\right): P \times_{B_{1}} \overline{\mathcal{M}}\left(B_{1}, B_{0}\right) \stackrel{\pi_{2}}{\longrightarrow} \overline{\mathcal{M}}\left(B_{1}, B_{0}\right) \stackrel{\partial_{+}}{\longrightarrow}\{n, s\}
$$

represents either $n-s \in C_{0}(f)$ or $s-n \in C_{0}(f)$, depending on the orientation chosen for $B_{1}$. (If we fix an orientation on $B_{1}$, then the sign is determined by Definition 5.7). Therefore,

$$
H_{k}\left(C_{*}(f), \partial\right)= \begin{cases}\mathbb{Z} & \text { if } k=0,2, \\ 0 & \text { otherwise. }\end{cases}
$$

5.5. Manifolds with corners. In this subsection we recall some facts about manifolds with corners, and we prove Lemmas 5.1 and 5.21. Note that the definition of the Morse-Bott-Smale chain complex does not rely on the smooth manifold with corners structure on the objects in $C_{p}$. All that is required (in addition to the abstract topological chain structure) is the notion of an orientation on these objects and some finiteness conditions. In fact, to define the Morse-Bott-Smale chain complex it would be sufficient to show that the objects in $C_{p}$ are oriented topological manifolds with boundary and that for any $P \in C_{p}$

$$
P \times_{B_{i}} \overline{\mathcal{M}}\left(B_{i}, B_{i-j}\right)
$$

has finitely many components.

However, the following example (adapted from [10]) shows that some additional structure is needed to guarantee that these fibered products have finitely many components.

Example 5.17. Let $f:[0,1] \rightarrow[0,1] \times[-1,1]$ be given by

$$
f(t)= \begin{cases}\left(t, e^{-1 / t^{2}} \sin (\pi / t)\right) & \text { if } t \neq 0 \\ (0,0) & \text { if } t=0\end{cases}
$$

and $g:[0,1] \times[0,1] \rightarrow[0,1] \times[-1,1]$ be given by $g(x, y)=(x, 0)$. Then $f$ and $g$ are smooth maps between finite-dimensional compact oriented topological manifolds 
with boundary whose fibered product

$$
[0,1] \times{ }_{(f, g)}[0,1] \times[0,1]=\{(t, t, 0) \in[0,1] \times[0,1] \times[0,1] \mid t=0,1,1 / 2,1 / 3, \ldots\}
$$

has infinitely many components.

Note that this example also shows that the fibered product of two finite CWcomplexes might not be a CW-complex, and the fibered product of two finite simplicial complexes might not be a finite simplicial complex. So, a category with some additional structure is needed in order to prove that the fibered products of interest for Morse-Bott homology have finitely many components. An analysis of the above example shows that the failure of this fibered product to have finitely many components is due to a lack of transversality. Thus, the category of compact smooth manifolds with corners is a good candidate for proving the required finiteness conditions. Other choices are possible, including the category of compact manifolds with corners of class $C^{s}$ where $s \geq 1$.

Recall that a manifold with corners of dimension $m$ is a Hausdorff topological space $X$ such that every point $x \in X$ has a neighborhood $U_{x} \subseteq X$ that is locally homeomorphic via a chart $\psi_{x}: U_{x} \rightarrow \mathbb{R}_{(r)}^{m}$ to some open subset of $\mathbb{R}_{(r)}^{m}=\mathbb{R}^{r} \times$ $[0, \infty)^{m-r}$. A manifold with corners is said to be of class $C^{s}$ if and only if for any two charts $\psi_{x}: U_{x} \rightarrow \mathbb{R}_{(r)}^{m}$ and $\psi_{y}: U_{y} \rightarrow \mathbb{R}_{(r)}^{m}$ the composition $\psi_{x} \circ \psi_{y}^{-1}$ : $\psi_{y}\left(U_{x} \cap U_{y}\right) \rightarrow \psi_{x}\left(U_{x} \cap U_{y}\right)$ extends to a map of class $C^{s}$ between open sets of $\mathbb{R}^{m}$. We will assume that $s \geq 1$, and if $s=\infty$, then we will call $X$ a smooth manifold with corners.

If $\psi_{x}: U_{x} \rightarrow \mathbb{R}_{(r)}^{m}$ is a local chart on $X$ such that $\psi_{x}(x)=0 \in \mathbb{R}_{(r)}^{m}$, then the number $r$ does not depend on the choice of the chart $\psi_{x}$ and is called the index of $x$ in $X$, denoted ind $(X, x)$. A manifold with corners $X$ is stratified by manifolds (without boundary) $X_{0}, X_{1}, \ldots, X_{m}$ defined by

$$
X_{r}=\{x \in X \mid \operatorname{ind}(X, x)=r\}
$$

for all $r=0, \ldots, m$. A connected component of $X_{r}$ is called an $r$-stratum of $X$, and a subset of $X$ that is an $r$-stratum for some $r$ is called a stratum of $X$. The set $X_{m}$ is the interior of $X$, and the set $X-X_{m}$ is called the boundary of $X$. For more details see [32, 34, or 38.

The strata of the compact smooth manifold with corners $\overline{\mathcal{M}}\left(B_{i}, B_{i-j}\right)$ can be described as follows. To simplify the notation assume that the components of $B_{i}$ are all of dimension $b_{i}$, which implies that $\overline{\mathcal{M}}\left(B_{i}, B_{i-j}\right)$ has dimension $m=b_{i}+j-1$. The strata are then the connected components of the sets

$$
\begin{aligned}
X_{m-r}= & \bigcup_{\substack{0<j_{1}<\cdots<j_{r}<j\\
}} \mathcal{M}\left(B_{i}, B_{i-j_{1}}\right) \times_{B_{i-j_{1}}} \mathcal{M}\left(B_{i-j_{1}}, B_{i-j_{2}}\right) \\
& \times_{B_{i-j_{2}}} \cdots \times_{B_{i-j_{r}}} \mathcal{M}\left(B_{i-j_{r}}, B_{i-j}\right)
\end{aligned}
$$

where $r=0, \ldots, j-1$, and the union is taken over all increasing sequences of integers $j_{1}<j_{2}<\cdots<j_{r}$ between 1 and $j-1$. Note that $X_{m}=\mathcal{M}\left(B_{i}, B_{i-j}\right)$, and if $1 \leq r \leq j-1$, then the set $X_{m-r}$ consists of those piecewise gradient flow lines that pass through exactly $r$ intermediate critical submanifolds.

We will use the following theorem (see Theorem 3 of [38]).

Theorem 5.18. Let $X$ and $Y$ be $C^{s}$ manifolds with corners, where $s \geq 1$. Let $A \subseteq Y$ be a $C^{s}$ submanifold with corners, and $f: X \rightarrow Y$ be a local $C^{s}$ map 
which preserves local facets relative to $A$ and intersects $A$ transversally and stratum transversally. Then either $f^{-1}(A)=\emptyset$ or

(1) $f^{-1}(A)$ is a $C^{s}$ submanifold with corners of $X$, and

(2) $\operatorname{dim} X-\operatorname{dim} f^{-1}(A)=\operatorname{dim} Y-\operatorname{dim} A$, and

(3) $\operatorname{ind}(X, x)-\operatorname{ind}\left(f^{-1}(A), x\right)=\operatorname{ind}(Y, f(x))-\operatorname{ind}(A, f(x))$ for all $x \in f^{-1}(A)$.

For a definition of "local facets" see [38. We will not define local facets here because we will only apply this theorem in the case where $Y$ is a manifold without boundary, and the local facets condition is always satisfied in that case. The assumption that $f$ intersects $A$ stratum transversally means that for any $x \in$ $f^{-1}(A)$ we have

$$
d f_{x}\left(\hat{T}_{x} X\right)+\hat{T}_{y} A=\hat{T}_{y} Y,
$$

where $y=f(x)$ and $\hat{T}_{x} X$ denotes the tangent space of the stratum containing $x \in X$. Similarly, we will say that a map $f: X \rightarrow Y$ is a stratum submersion at $x \in X$ if and only if $d f_{x}$ maps $\hat{T}_{x} X$ onto $\hat{T}_{y} Y$ where $y=f(x)$. Note that if $f$ is a stratum submersion at $x \in X$ and $A \subseteq Y$ is any submanifold with corners containing $y$, then $f$ intersects $A$ stratum transversally.

Lemma 5.19. For any two connected critical submanifolds $B$ and $B^{\prime}$, the beginning point map

$$
\partial_{-}: \mathcal{M}\left(B, B^{\prime}\right) \rightarrow B
$$

is a submersion.

Proof. Let $B$ be a connected critical submanifold of a Morse-Bott-Smale function $f: M \rightarrow \mathbb{R}$ of dimension $b$ and index $\lambda_{B}$. By Theorem 3.3 the beginning point map

$$
\partial_{-}: W^{u}(B) \rightarrow B
$$

is a locally trivial fiber bundle, and if $B^{\prime}$ is any other critical submanifold, then either $W\left(B, B^{\prime}\right)=\emptyset$ (in which case the lemma holds trivially) or $W\left(B, B^{\prime}\right)$ is a manifold of dimension $\lambda_{B}-\lambda_{B^{\prime}}+b$ (by Lemma 3.5).

Let $x \in B$ and let $U \subset B$ be a coordinate neighborhood of $x$ such that the bundle $\partial_{-}: W^{u}(B) \rightarrow B$ is trivial over $U$. We can extend the coordinate chart $U$ on $B$ to a coordinate chart $V$ on $M$ so that in $V$ we have local coordinates

$$
\mathbb{R}^{b} \times \mathbb{R}^{\lambda_{B}} \times \mathbb{R}^{m-b-\lambda_{B}},
$$

where $\mathbb{R}^{b}$ represents the coordinates on $B$ and $\mathbb{R}^{\lambda_{B}}$ represents the coordinates along the fiber of $W^{u}(B)$. In these local coordinates the beginning point map $\partial_{-}$: $W^{u}(U) \rightarrow U$ is given by the projection map

$$
\pi: \mathbb{R}^{b} \times \mathbb{R}^{\lambda_{B}} \times \overrightarrow{0} \rightarrow \mathbb{R}^{b} \times \overrightarrow{0} \times \overrightarrow{0} .
$$

Let $\tilde{x} \in V$ be a point in the fiber above $x$, i.e. $\partial_{-}(\tilde{x})=x$, such that $\tilde{x} \in W^{s}\left(B^{\prime}\right)$. In the local coordinates we have $T_{\tilde{x}} W^{u}(x)=\overrightarrow{0} \times \mathbb{R}^{\lambda_{B}} \times \overrightarrow{0}$, and the Morse-Bott-Smale transversality condition

$$
\left(\overrightarrow{0} \times \mathbb{R}^{\lambda_{B}} \times \overrightarrow{0}\right)+T_{\tilde{x}} W^{s}\left(B^{\prime}\right)=\mathbb{R}^{m} \approx T_{\tilde{x}} M
$$

implies that we can find a smooth section $s: U^{\prime} \rightarrow V$ of the bundle $\partial_{-}: W^{u}(B) \rightarrow$ $B$ on an open set $U^{\prime} \subseteq U$ containing $x$ such that $s(x)=\tilde{x}$ and $s(z) \in W^{s}\left(B^{\prime}\right)$ for all $z \in U^{\prime}$. The image of the section $s: U^{\prime} \rightarrow V$ is in $W\left(B, B^{\prime}\right)=W^{u}(B) \cap W^{s}\left(B^{\prime}\right)$ 
by construction, and diffeomorphic to the open set $U^{\prime} \subseteq B$ via the beginning point map $\partial_{-}: W^{u}\left(U^{\prime}\right) \rightarrow U^{\prime}$. This shows that the beginning point map

$$
\partial_{-}: W\left(B, B^{\prime}\right) \rightarrow B
$$

is a submersion at $\tilde{x}$, and using the diffeomorphisms determined by the gradient flow we see that the beginning point map is a submersion at every point $\tilde{x} \in W\left(B, B^{\prime}\right)$.

If we pick an appropriate level set $f^{-1}(a)$ between $B$ and $B^{\prime}$, then we can identify

$$
\mathcal{M}\left(B, B^{\prime}\right)=W\left(B, B^{\prime}\right) \cap f^{-1}(a) .
$$

This shows that

$$
\partial_{-}: \mathcal{M}\left(B, B^{\prime}\right) \rightarrow B
$$

is a submersion because the level sets are transverse to the gradient flow.

Corollary 5.20. For any two connected critical submanifolds $B$ and $B^{\prime}$, the beginning point map

$$
\partial_{-}: \overline{\mathcal{M}}\left(B, B^{\prime}\right) \rightarrow B
$$

is a submersion and a stratum submersion.

Proof. Since $B$ is a manifold without boundary, $\hat{T}_{y} B=T_{y} B$ for all $y \in B$. Hence, it suffices to show that $\partial_{-}: \overline{\mathcal{M}}\left(B, B^{\prime}\right) \rightarrow B$ is a stratum submersion. The boundary of $\overline{\mathcal{M}}\left(B, B^{\prime}\right)$ is stratified by fibered products of moduli spaces of gradient flow lines of the form

$$
\mathcal{M}\left(B, B_{1}\right) \times_{B_{1}} \mathcal{M}\left(B_{1}, B_{2}\right) \times_{B_{2}} \cdots \times_{B_{n}} \mathcal{M}\left(B_{n}, B^{\prime}\right)
$$

for some intermediate critical submanifolds $B_{1}, \ldots, B_{n}$. To see that these strata are smooth manifolds without boundary, assume for the purpose of induction that

$$
\mathcal{M}\left(B, B_{1}\right) \times_{B_{1}} \mathcal{M}\left(B_{1}, B_{2}\right) \times_{B_{2}} \cdots \times_{B_{n-1}} \mathcal{M}\left(B_{n-1}, B_{n}\right)
$$

is a smooth manifold without boundary. By Lemma 5.19 the map $\partial_{-}: \mathcal{M}\left(B_{n}, B^{\prime}\right) \rightarrow$ $B_{n}$ is a submersion, and hence

$$
\mathcal{M}\left(B, B_{1}\right) \times_{B_{1}} \mathcal{M}\left(B_{1}, B_{2}\right) \times_{B_{2}} \cdots \times_{B_{n}} \mathcal{M}\left(B_{n}, B^{\prime}\right)
$$

is a smooth manifold without boundary by Lemma 4.5.

Now let $P=\mathcal{M}\left(B_{1}, B_{2}\right) \times_{B_{2}} \cdots \times_{B_{n}} \mathcal{M}\left(B_{n}, B^{\prime}\right)$ and assume for the purpose of induction that $\partial_{-}: P \rightarrow B_{1}$ is a submersion. This assumption implies that the projection

$$
\pi_{1}: \mathcal{M}\left(B, B_{1}\right) \times_{B_{1}} P \rightarrow \mathcal{M}\left(B, B_{1}\right)
$$

is a submersion. To see this, let $v \in T_{x} \mathcal{M}\left(B, B_{1}\right)$, and let $\alpha:(-\varepsilon, \varepsilon) \rightarrow \mathcal{M}\left(B, B_{1}\right)$ be a smooth curve such that $\alpha(0)=x$ and $\alpha^{\prime}(0)=v$. Since $\partial_{-}: P \rightarrow B_{1}$ is a submersion, it is locally a projection map. Hence, if $\partial_{+}(\alpha(0)) \in B_{1}$ is contained in the image of $\partial_{-}: P \rightarrow B_{1}$, then for $t$ sufficiently small we have $\partial_{+}(\alpha(t)) \in$ $\partial_{-}(P)$. This implies that we can find a smooth curve $\gamma:(-\varepsilon, \varepsilon) \rightarrow P$ such that $\partial_{+}(\alpha(t))=\partial_{-}(\gamma(t))$ for $t$ sufficiently small. The smooth curve $\alpha \times \gamma$ then lies in $\mathcal{M}\left(B, B_{1}\right) \times_{B_{1}} P$ for $t$ sufficiently small and satisfies $d \pi_{1}\left(\alpha^{\prime}(0) \times \gamma^{\prime}(0)\right)=v$.

To complete the proof of the corollary, simply note that $\partial_{-}: \mathcal{M}\left(B, B_{1}\right) \rightarrow B$ is a submersion by Lemma 5.19 and hence the composition of this map with $\pi_{1}$ :

$$
\partial_{-}: \mathcal{M}\left(B, B_{1}\right) \times_{B_{1}} \mathcal{M}\left(B_{1}, B_{2}\right) \times_{B_{2}} \cdots \times_{B_{n}} \mathcal{M}\left(B_{n}, B^{\prime}\right) \rightarrow B
$$

is also a submersion. 
Lemma 5.21. If $B$ and $B^{\prime}$ are connected critical submanifolds and $\sigma: P \rightarrow B$ is a smooth map from a compact smooth manifold with corners $P$, then

$$
P \times_{B} \overline{\mathcal{M}}\left(B, B^{\prime}\right)
$$

is a compact smooth manifold with corners.

Proof. Let $\Delta=\{(b, b) \mid b \in B\}$ be the diagonal in $B \times B$. By Corollary [5.20 the beginning point map $\partial_{-}: \overline{\mathcal{M}}\left(B, B^{\prime}\right) \rightarrow B$ is a submersion and a stratum submersion. Hence, the map $\sigma \times \partial_{-}: P \times \overline{\mathcal{M}}\left(B, B^{\prime}\right) \rightarrow B \times B$ is transverse and stratum transverse to the submanifold $\Delta \subset B \times B$. Thus,

$$
P \times_{B} \overline{\mathcal{M}}\left(B, B^{\prime}\right)=\left(\sigma \times \partial_{-}\right)^{-1}(\Delta)
$$

is a manifold with corners by Theorem 5.18. The space $P \times_{B} \overline{\mathcal{M}}\left(B, B^{\prime}\right)$ is compact because it is a closed subset of the compact space $P \times \overline{\mathcal{M}}\left(B, B^{\prime}\right)$.

Proof of Lemma 5.1. The faces of $I^{N}$ are obviously compact smooth manifolds with corners, and for any two connected critical submanifolds $B$ and $B^{\prime}$ the compactified moduli space $\overline{\mathcal{M}}\left(B, B^{\prime}\right)$ is a compact smooth manifolds with corners by Theorem 4.9. The rest of the elements in $C_{p}$ are constructed inductively from these elements, and hence Lemma 5.21 can be applied inductively to show that all the elements of $C_{p}$ are compact smooth manifolds with corners. The orientations on these manifolds were defined previously.

\section{INDEPENDENCE OF THE FUNCTION}

In this section we prove that the homology of the Morse-Bott chain complex $\left(C_{*}(f), \partial\right)$ is independent of the Morse-Bott-Smale function $f$, and hence is isomorphic to the singular homology of $M$ with integer coefficients. The method of proof follows standard arguments found in 4, 49, 18, 19, 41, and [44. The outline of the proof is as follows.

Given two Morse-Bott-Smale functions $f_{1}, f_{2}: M \rightarrow \mathbb{R}$ we pick a smooth function $F_{21}: M \times \mathbb{R} \rightarrow \mathbb{R}$ meeting certain transversality requirements such that

$$
\begin{aligned}
\lim _{t \rightarrow-\infty} F_{21}(x, t) & =f_{1}(x)+1, \\
\lim _{t \rightarrow+\infty} F_{21}(x, t) & =f_{2}(x)-1
\end{aligned}
$$

for all $x \in M$. The compactified moduli spaces of gradient flow lines of $F_{21}$ (the time dependent gradient flow lines) are used to define a chain map $\left(F_{21}\right)_{\square}: C_{*}\left(f_{1}\right) \rightarrow$ $C_{*}\left(f_{2}\right)$, where $\left(C_{*}\left(f_{k}\right), \partial\right)$ is the Morse-Bott chain complex of $f_{k}$ for $k=1,2$.

Next we consider the case where we have four Morse-Bott-Smale functions $f_{k}$ : $M \rightarrow \mathbb{R}$ where $k=1,2,3,4$, and we pick a smooth function $H: M \times \mathbb{R} \times \mathbb{R} \rightarrow \mathbb{R}$ meeting certain transversality requirements such that

$$
\begin{aligned}
\lim _{s \rightarrow-\infty} \lim _{t \rightarrow-\infty} H(x, s, t) & =f_{1}(x)+2, \\
\lim _{s \rightarrow+\infty} \lim _{t \rightarrow-\infty} H(x, s, t) & =f_{2}(x), \\
\lim _{s \rightarrow-\infty} \lim _{t \rightarrow+\infty} H(x, s, t) & =f_{3}(x), \\
\lim _{s \rightarrow+\infty} \lim _{t \rightarrow+\infty} H(x, s, t) & =f_{4}(x)-2
\end{aligned}
$$


for all $x \in M$. The compactified moduli spaces of gradient flow lines of $H$ are used to define a chain homotopy between $\left(F_{43}\right) \square \circ\left(F_{31}\right) \square$ and $\left(F_{42}\right)_{\square} \circ\left(F_{21}\right) \square$, where $\left(F_{l k}\right) \square: C_{*}\left(f_{k}\right) \rightarrow C_{*}\left(f_{l}\right)$ is the map defined above for $k, l=1,2,3,4$. In homology the map $\left(F_{k k}\right)_{*}: H_{*}\left(C_{*}\left(f_{k}\right), \partial\right) \rightarrow H_{*}\left(C_{*}\left(f_{k}\right), \partial\right)$ is the identity for all $k$, and hence

$$
\begin{aligned}
& \left(F_{12}\right)_{*} \circ\left(F_{21}\right)_{*}=\left(F_{11}\right)_{*} \circ\left(F_{11}\right)_{*}=i d, \\
& \left(F_{21}\right)_{*} \circ\left(F_{12}\right)_{*}=\left(F_{22}\right)_{*} \circ\left(F_{22}\right)_{*}=i d .
\end{aligned}
$$

Therefore,

$$
\left(F_{21}\right)_{*}: H_{*}\left(C_{*}\left(f_{1}\right), \partial\right) \rightarrow H_{*}\left(C_{*}\left(f_{2}\right), \partial\right)
$$

is an isomorphism.

6.1. Time dependent gradient flow lines. Let $\rho: \mathbb{R} \rightarrow(-1,1)$ be a smooth strictly increasing function such that $\lim _{t \rightarrow-\infty} \rho(t)=-1$ and $\lim _{t \rightarrow+\infty} \rho(t)=1$. The function $\rho$ determines a function on the compactified real line $\overline{\mathbb{R}}=\mathbb{R} \cup\{ \pm \infty\}$ by defining $\rho(-\infty)=-1$ and $\rho(+\infty)=1$. (The compactified real line $\overline{\mathbb{R}}$ is a compact smooth manifold with boundary with a single chart $h: \overline{\mathbb{R}} \rightarrow[-1,1]$ given by $h(t)=\frac{t}{\sqrt{1+t^{2}}}$; see Definition 2.1 of [41]. Note that we could choose $\rho=h$.)

Let $f_{1}, f_{2}: M \rightarrow \mathbb{R}$ be Morse-Bott-Smale functions, and let $B_{i}^{f_{1}}$ and $B_{j}^{f_{2}}$ denote the set of critical points of $f_{1}$ and $f_{2}$ respectively of index $i, j=0, \ldots, m$. Let $F_{21}: M \times \mathbb{R} \rightarrow \mathbb{R}$ be a smooth function that is strictly decreasing in its second component such that for some large $T \gg 0$ we have

$$
F_{21}(x, t)=\left\{\begin{array}{llc}
f_{1}(x)-\rho(t) & \text { if } & t<-T, \\
h_{t}(x) & \text { if } & -T \leq t \leq T, \\
f_{2}(x)-\rho(t) & \text { if } & t>T,
\end{array}\right.
$$

where $h_{t}(x)$ is an approximation to $\frac{1}{2}(T-t)\left(f_{1}(x)-\rho(t)\right)+\frac{1}{2}(T+t)\left(f_{2}(x)-\rho(t)\right)$ that makes $F_{21}$ smooth. (To simplify the notation in the following computations we will sometimes drop the subscript on $F_{12}$.)

Let $\varphi_{\alpha}: M \times \mathbb{R} \rightarrow M \times \mathbb{R}$ denote the flow associated to $-\nabla F_{21}$ with respect to the product metric on $M \times \mathbb{R}$. Thus, $\varphi_{\alpha}(x, t)=(\gamma(\alpha), \sigma(\alpha)) \in M \times \mathbb{R}$, where

$$
\left(\gamma^{\prime}(\alpha), \sigma^{\prime}(\alpha)\right)= \begin{cases}\left(-\left(\nabla f_{1}\right)(\gamma(\alpha)), \rho^{\prime}(\alpha)\right) & \text { if } t<-T \\ \left(-\left(\nabla f_{2}\right)(\gamma(\alpha)), \rho^{\prime}(\alpha)\right) & \text { if } t>T\end{cases}
$$

and $(\gamma(0), \sigma(0))=(x, t)$. The negative gradient flow $\varphi_{\alpha}: M \times \mathbb{R} \rightarrow M \times \mathbb{R}$ of $F_{21}$ determines stable and unstable manifolds in $M \times \mathbb{R}$ of the critical submanifolds of $f_{1}$ and $f_{2}$ :

$$
\begin{aligned}
& W_{F}^{s}\left(B_{j}^{f_{2}}\right)=\left\{(x, t) \in M \times \mathbb{R} \mid \lim _{\alpha \rightarrow+\infty} \pi_{1}\left(\varphi_{\alpha}(x, t)\right) \in B_{j}^{f_{2}}\right\}, \\
& W_{F}^{u}\left(B_{i}^{f_{1}}\right)=\left\{(x, t) \in M \times \mathbb{R} \mid \lim _{\alpha \rightarrow-\infty} \pi_{1}\left(\varphi_{\alpha}(x, t)\right) \in B_{i}^{f_{1}}\right\},
\end{aligned}
$$

where $\pi_{1}: M \times \mathbb{R} \rightarrow M$ denotes projection onto the first component. These stable and unstable manifolds inherit orientations from the stable and unstable manifolds of $f_{1}$ and $f_{2}$, and since both $f_{1}$ and $f_{2}$ satisfy the Morse-Bott-Smale transversality condition, we can choose the approximation $h_{t}(x)$ so that $W_{F}^{u}\left(B_{i}^{f_{1}}\right) \pitchfork W_{F}^{s}\left(B_{j}^{f_{2}}\right)$ is an oriented submanifold of $M \times \mathbb{R}$ for all $i, j=0, \ldots, m$. 
Definition 6.1. The moduli spaces of gradient flow lines of $F_{21}$ (the time dependent gradient flow lines) are defined to be

$$
\mathcal{M}_{F}\left(B_{i}^{f_{1}}, B_{j}^{f_{2}}\right)=\left(W_{F}^{u}\left(B_{i}^{f_{1}}\right) \cap W_{F}^{s}\left(B_{j}^{f_{2}}\right)\right) / \mathbb{R}
$$

for all $i, j=0, \ldots, m$, where the $\mathbb{R}$-action is defined by the negative gradient flow.

Lemma 6.2. For a generic function $F_{21}$ satisfying the conditions listed above, the moduli space $\mathcal{M}_{F}\left(B_{i}^{f_{1}}, B_{j}^{f_{2}}\right)$ is either empty or an oriented smooth manifold of dimension $b_{i}^{f_{1}}+i-j$.

Proof. The dimension of $W_{F}^{u}\left(B_{i}^{f_{1}}\right)$ is $b_{i}^{f_{1}}+i+1$, and the dimension of $W_{F}^{s}\left(B_{j}^{f_{2}}\right)$ is $m+1-j$. Thus, the dimension of the transverse intersection $W_{F}^{u}\left(B_{i}^{f_{1}}\right) \pitchfork W_{F}^{s}\left(B_{j}^{f_{2}}\right)$ is

$$
\left(b_{i}^{f_{1}}+i+1\right)+(m+1-j)-(m+1)=b_{i}^{f_{1}}+i-j+1 .
$$

Note. The preceding lemma implies that $\mathcal{M}_{F}\left(B_{i}^{f_{1}}, B_{j}^{f_{2}}\right)=\emptyset$ whenever $b_{i}^{f_{1}}+i<j$. This is a weaker statement than the one found in Lemma 3.6, where the function is assumed to satisfy the Morse-Bott-Smale transversality condition.

There are gluing and compactification results for time dependent gradient flow lines analogous to Theorems 4.8 and 4.9. In order to distinguish between the time independent gradient flow lines and the time dependent gradient flow lines, we will denote the moduli spaces of (time independent) gradient flow lines of $f_{1}$ by $\mathcal{M}_{f_{1}}\left(B_{i}^{f_{1}}, B_{j}^{f_{1}}\right)$. Similarly, we will denote the (time independent) moduli spaces of gradient flow lines of $f_{2}$ by $\mathcal{M}_{f_{2}}\left(B_{i}^{f_{2}}, B_{j}^{f_{2}}\right)$. The proofs of the following two fundamental theorems follow from the results in Appendix $\S \mathrm{A} .2$ and $\S \mathrm{A} .3$ of 4 . See [13, 27, and 26] for an alternate approach to these theorems.

Theorem 6.3 (Gluing). For large $N \gg 0$ there are injective local diffeomorphisms

$$
\begin{aligned}
& G_{L}: \mathcal{M}_{f_{1}}\left(B_{i}^{f_{1}}, B_{n}^{f_{1}}\right) \times_{B_{n}^{f_{1}}} \mathcal{M}_{F}\left(B_{n}^{f_{1}}, B_{j}^{f_{2}}\right) \times(N, \infty) \rightarrow \mathcal{M}_{F}\left(B_{i}^{f_{1}}, B_{j}^{f_{2}}\right), \\
& G_{R}: \mathcal{M}_{F}\left(B_{i}^{f_{1}}, B_{n}^{f_{2}}\right) \times_{B_{n}^{f_{2}}} \mathcal{M}_{f_{2}}\left(B_{n}^{f_{2}}, B_{j}^{f_{2}}\right) \times(N, \infty) \rightarrow \mathcal{M}_{F}\left(B_{i}^{f_{1}}, B_{j}^{f_{2}}\right)
\end{aligned}
$$

onto the open ends of $\mathcal{M}_{F}\left(B_{i}^{f_{1}}, B_{j}^{f_{2}}\right)$.

Theorem 6.4 (Compactification). The moduli space $\mathcal{M}_{F}\left(B_{i}^{f_{1}}, B_{j}^{f_{2}}\right)$ has a compactification $\overline{\mathcal{M}}_{F}\left(B_{i}^{f_{1}}, B_{j}^{f_{2}}\right)$, consisting of all the piecewise gradient flow lines from $B_{i}^{f_{1}}$ to $B_{j}^{f_{2}}$ (including both time dependent and time independent gradient flow lines), which is either empty or a compact oriented smooth manifold with corners of dimension $b_{i}^{f_{1}}+i-j$. The beginning and endpoint maps extend to smooth maps on the compactified spaces.

The compactification of the moduli spaces of time dependent gradient flow lines by piecewise gradient flow lines determines a degree and boundary operator that satisfies the axioms for abstract topological chains. To simplify the notation we will assume that for each $i=0, \ldots, m$ the components of $B_{i}^{f_{1}}$ are of the same dimension. In general one needs to group the components by their dimension and then define the degree and boundary operator on each group. 
Definition 6.5. The degree of $\overline{\mathcal{M}}_{F}\left(B_{i}^{f_{1}}, B_{j}^{f_{2}}\right)$ is defined to be $b_{i}^{f_{1}}+i-j$, and the boundary operator $\partial \overline{\mathcal{M}}_{F}\left(B_{i}^{f_{1}}, B_{j}^{f_{2}}\right)$ is defined to be $(-1)^{i+b_{i}^{f_{1}}}$ times the quantity

$$
\begin{aligned}
\left(\sum_{n<i} \overline{\mathcal{M}}_{f_{1}}\left(B_{i}^{f_{1}}, B_{n}^{f_{1}}\right) \times_{B_{n}^{f_{1}}} \overline{\mathcal{M}}_{F}\left(B_{n}^{f_{1}}, B_{j}^{f_{2}}\right)\right. & \\
& \left.-\sum_{j<n} \overline{\mathcal{M}}_{F}\left(B_{i}^{f_{1}}, B_{n}^{f_{2}}\right) \times_{B_{n}^{f_{2}}} \overline{\mathcal{M}}_{f_{2}}\left(B_{n}^{f_{2}}, B_{j}^{f_{2}}\right)\right),
\end{aligned}
$$

where $b_{i}^{f_{1}}=\operatorname{dim} B_{i}^{f_{1}}$ and the fibered products are taken over the beginning and endpoint maps $\partial_{-}$and $\partial_{+}$. If $B_{n}=\emptyset$, then the corresponding compactified moduli spaces and fibered products are identified with zero. The boundary operator extends to fibered products of compactified moduli spaces via Definition 4.6 .

The proof of the following lemma is analogous to the proof of Lemma 4.12

Lemma 6.6. The degree and boundary operator for $\overline{\mathcal{M}}_{F}\left(B_{i}^{f_{1}}, B_{j}^{f_{2}}\right)$ satisfy the axioms for abstract topological chains, i.e. the boundary operator is of degree -1 and satisfies $\partial \circ \partial=0$.

6.2. Representing chain systems. Intuitively, the chain map $\left(F_{21}\right) \square: \tilde{C}_{*}\left(f_{1}\right) \rightarrow$ $\tilde{C}_{*}\left(f_{2}\right)$ should be defined as follows. Given a singular $C_{p}^{f_{1}}$-space $\sigma: P \rightarrow B_{i}^{f_{1}}$ that is transverse and stratum transverse to $\partial_{-}: \overline{\mathcal{M}}_{F}\left(B_{i}^{f_{1}}, B_{j}^{f_{2}}\right) \rightarrow B_{i}^{f_{1}}$ for all $j=0, \ldots, m$, we would like to define

$$
\left(F_{21}\right)_{\square}(\sigma)=\bigoplus_{j=0}^{m}\left(\partial_{+} \circ \pi_{2}: P \times_{B_{i}^{f_{1}}} \overline{\mathcal{M}}_{F}\left(B_{i}^{f_{1}}, B_{j}^{f_{2}}\right) \rightarrow B_{j}^{f_{2}}\right) \in \bigoplus_{j=0}^{m} S_{p+i-j}^{\infty}\left(B_{j}^{f_{2}}\right),
$$

where $P \times_{B_{i}^{f_{1}}} \overline{\mathcal{M}}_{F}\left(B_{i}^{f_{1}}, B_{j}^{f_{2}}\right) \stackrel{\pi_{2}}{\longrightarrow} \overline{\mathcal{M}}_{F}\left(B_{i}^{f_{1}}, B_{j}^{f_{2}}\right) \stackrel{\partial_{+}}{\longrightarrow} B_{j}^{f_{2}}$ is defined as in Lemma 5.3. However, fibered products of the compactified moduli spaces of time dependent gradient flow lines with singular cubes in the critical submanifolds of $f_{1}$ are not elements of the sets $\left\{C_{p}^{f_{2}}\right\}_{p \geq 0}$ of allowed domains for the singular topological chains in $\tilde{C}_{*}\left(f_{2}\right)$. So, to define the map rigorously we need to choose a topological chain in $S_{p+i-j}^{\infty}\left(B_{j}^{f_{2}}\right)$ that represents the map $\partial_{+} \circ \pi_{2}: P \times_{B_{i}^{f_{1}}} \overline{\mathcal{M}}_{F}\left(B_{i}^{f_{1}}, B_{j}^{f_{2}}\right) \rightarrow B_{j}^{f_{2}}$. To do this we adapt the idea of representing chain systems from [9] to our setup.

For every $p \geq 0$ let $C_{p}^{F}$ be the set of $p$-dimensional components of the fibered products of the compactified moduli spaces of time dependent gradient flow lines of $F_{21}$ with elements of $\tilde{C}_{*}\left(f_{1}\right)$ that intersect the $\partial_{-}$maps transversally and stratum transversally, and let $S_{p}^{F}$ be the free abelian group generated by the elements of $C_{p}^{F}$, i.e. $S_{p}^{F}=\mathbb{Z}\left[C_{p}^{F}\right]$. Note that for any $p \geq 0$ the elements of $C_{p}^{F}$ are compact oriented smooth manifolds with corners, and hence they have fundamental classes in relative homology. Moreover, $\left(S_{*}^{F}, \partial\right)$ is a chain complex of abstract topological chains by Lemma 4.7

Definition 6.7. A representing chain system for $\left\{C_{p}^{F}\right\}_{p \geq 0}$ is a family of smooth singular cubical chains $\left\{s_{P} \in S_{p}(P) \mid P \in C_{p}^{F}\right\}_{p \geq 0}$ such that

(1) The image of the smooth singular cubical chain $s_{P}$ in the relative cubical chain group $S_{p}(P, \partial P)=S_{p}(P) / S_{p}(\partial P)$ (where $\partial P$ denotes the topological boundary of $P$ ) is a cycle that represents the fundamental class of $P$. 
(2) If $\partial P=\sum_{k} n_{k} P_{k} \in S_{p-1}^{F}$ where $n_{k}= \pm 1$ and $P_{k} \in C_{p-1}^{F}$, then

$$
\partial s_{P}=\sum_{k} n_{k} s_{P_{k}} \in S_{p-1}(P),
$$

where $\partial s_{P}$ denotes the boundary operator on smooth singular cubical chains.

Lemma 6.8. There exists a representing chain system for $\left\{C_{p}^{F}\right\}_{p \geq 0}$.

Proof. The proof is by induction on the degree $p$. For $p=0$ the statement is obvious. So, assume that we have chosen representing chain systems for all the elements in $C_{j}^{F}$ where $j<p$, and let $P \in C_{p}^{F}$. Consider the following diagram:

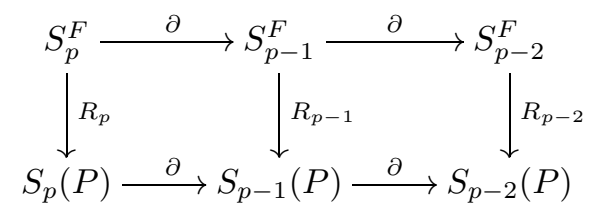

where $R_{p-1}$ and $R_{p-2}$ are defined by sending linear combinations of subsets of $P$ in $S_{p-1}^{F}$ and $S_{p-2}^{F}$ to their representing chains and all other elements to zero.

If $\partial P=\sum_{k} n_{k} P_{k} \in S_{p-1}^{F}$, then by the induction hypothesis $R_{p-1}(\partial P)=$ $\sum_{k} n_{k} s_{P_{k}}$, where $s_{P_{k}} \in S_{p-1}\left(P_{k}\right) \subseteq S_{p-1}(P)$ is a smooth singular cubical chain whose image in $S_{p-1}\left(P_{k}, \partial P_{k}\right)$ is a cycle that represents the fundamental class of $P_{k}$. Therefore, we can choose an element $s_{P} \in S_{p} P$ such that $\partial s_{P}=\sum_{k} n_{k} s_{P_{k}}$ and the image of $s_{P}$ in $S_{p}(P, \partial P)$ represents the fundamental class of $P$. (See for instance Lemma VI.9.1 of [14.) Define $R_{p}(P)=s_{P}$.

6.3. The chain map $\left(F_{21}\right)_{\square}: C_{*}\left(f_{1}\right) \rightarrow C_{*}\left(f_{2}\right)$. Assume that a representing chain system $\left\{s_{P} \in S_{p}(P) \mid P \in C_{p}^{F}\right\}_{p \geq 0}$ has been chosen. The map $\left(F_{21}\right)_{\square}: \tilde{C}_{*}\left(f_{1}\right) \rightarrow$ $\tilde{C}_{*}\left(f_{2}\right)$ is defined as follows.

Definition 6.9. If $\sigma: P \rightarrow B_{i}^{f_{1}}$ is a singular $C_{p}^{f_{1}}$-space in $B_{i}^{f_{1}}$ that intersects the beginning point map $\partial_{-}: \overline{\mathcal{M}}_{F}\left(B_{i}^{f_{1}}, B_{j}^{f_{2}}\right) \rightarrow B_{i}^{f_{1}}$ transversally and stratum transversally, then define

$$
\left(F_{21}\right)_{\square}(\sigma)=\bigoplus_{j=0}^{m} F_{21}^{j}(\sigma) \in \bigoplus_{j=0}^{m} S_{p+i-j}^{\infty}\left(B_{j}^{f_{2}}\right),
$$

where

$$
F_{21}^{j}(\sigma)=\sum_{k} n_{k}\left(\partial_{+} \circ \pi_{2} \circ s_{k}\right)
$$

and

$$
\sum_{k} n_{k} s_{k} \in S_{p+i-j}\left(P \times_{B_{i}^{f_{1}}} \overline{\mathcal{M}}_{F}\left(B_{i}^{f_{1}}, B_{j}^{f_{2}}\right)\right)
$$

is the representing chain for $P \times{ }_{B_{i}^{f_{1}}} \overline{\mathcal{M}}_{F}\left(B_{i}^{f_{1}}, B_{j}^{f_{2}}\right)$ if $P \times_{B_{i}^{f_{1}}} \overline{\mathcal{M}}_{F}\left(B_{i}^{f_{1}}, B_{j}^{f_{2}}\right) \neq \emptyset$ and $F_{21}^{j}(\sigma)=0$ otherwise. 
Note. By general transversality arguments we may assume without loss of generality that the map $\sigma: P \rightarrow B_{i}^{f_{1}}$ intersects the beginning point map $\partial_{-}$: $\overline{\mathcal{M}}_{F}\left(B_{i}^{f_{1}}, B_{j}^{f_{2}}\right) \rightarrow B_{i}^{f_{1}}$ transversally and stratum transversally. Also, to simplify the notation we will suppress the representing chain systems and denote the chain $F_{21}^{j}(\sigma) \in S_{p+i-j}^{\infty}\left(B_{j}^{f_{2}}\right)$ in the preceding definition by

$$
\partial_{+} \circ \pi_{2}: P \times_{B_{i}^{f_{1}}} \overline{\mathcal{M}}_{F}\left(B_{i}^{f_{1}}, B_{j}^{f_{2}}\right) \rightarrow B_{j}^{f_{2}} .
$$

The proof of the following lemma is similar to the proof of Lemma 5.10 ,

Lemma 6.10. The map $\left(F_{21}\right)_{\square}: \tilde{C}_{*}\left(f_{1}\right) \rightarrow \tilde{C}_{*}\left(f_{2}\right)$ induces a map $\left(F_{21}\right)_{\square}:$ $C_{*}\left(f_{1}\right) \rightarrow C_{*}\left(f_{2}\right)$.

Proposition 6.11. The map $\left(F_{21}\right)_{\square}: C_{*}\left(f_{1}\right) \rightarrow C_{*}\left(f_{2}\right)$ is a chain map of degree zero. That is, the map preserves degree and $\partial \circ\left(F_{21}\right)_{\square}=\left(F_{21}\right)_{\square} \circ \partial$.

Proof. Let $\sigma: P \rightarrow B_{i}^{f_{1}}$ be a singular $C_{p}^{f_{1}}$-space in $B_{i}^{f_{1}}$ that intersects the beginning point map $\partial_{-}: \overline{\mathcal{M}}_{F}\left(B_{i}^{f_{1}}, B_{j}^{f_{2}}\right) \rightarrow B_{i}^{f_{1}}$ transversally and stratum transversally. The dimension of $P \times_{B_{i}^{f_{1}}} \overline{\mathcal{M}}_{F}\left(B_{i}^{f_{1}}, B_{j}^{f_{2}}\right)$ is $p+i-j$ by Lemma 4.5 and Theorem 6.4. and thus $\partial_{+} \circ \pi_{2}: P \times_{B_{i}^{f_{1}}} \overline{\mathcal{M}}_{F}\left(B_{i}^{f_{1}}, B_{j}^{f_{2}}\right) \rightarrow B_{j}^{f_{2}}$ has Morse-Bott degree $p+i$ for all $j=0, \ldots, m$. Therefore, the map $\left(F_{21}\right)_{\square}: C_{*}\left(f_{1}\right) \rightarrow C_{*}\left(f_{2}\right)$ preserves degree.

We will now show that $\left(F_{21}\right) \square(\partial(\sigma))=\partial\left(F_{21}\right) \square(\sigma) \in \tilde{C}_{*}\left(f_{2}\right)$. Thus $\left(F_{21}\right) \square(\partial(\sigma))$ $=\partial\left(F_{21}\right) \square(\sigma) \in C_{*}\left(f_{2}\right)$ by Lemma 5.10 and Lemma 6.10. To simplify the notation we give the following computation in terms of abstract topological chains and assume that for every $i=0, \ldots, m$ all the components of $B_{i}^{f_{1}}$ are of the same dimension $b_{i}^{f_{1}}$. In general the components need to be grouped by their dimensions and the computation repeated on each group. The abstract topological chain associated to $\partial(\sigma) \in C_{p+i-1}\left(f_{1}\right)$ is

$$
(-1)^{p+i} \partial P+\sum_{k<i} P \times_{B_{i}^{f_{1}}} \overline{\mathcal{M}}_{f_{1}}\left(B_{i}^{f_{1}}, B_{k}^{f_{1}}\right) .
$$

Hence, by Definition 6.9 we have

$$
\begin{aligned}
\left(F_{21}\right)_{\square}(\partial(\sigma))= & (-1)^{p+i} \sum_{j=0}^{m} \partial P \times_{B_{i}^{f_{1}}} \overline{\mathcal{M}}_{F}\left(B_{i}^{f_{1}}, B_{j}^{f_{2}}\right) \\
& +\sum_{j=0}^{m} \sum_{k<i} P \times_{B_{i}^{f_{1}}} \overline{\mathcal{M}}_{f_{1}}\left(B_{i}^{f_{1}}, B_{k}^{f_{1}}\right) \times_{B_{k}^{f_{1}}} \overline{\mathcal{M}}_{F}\left(B_{k}^{f_{1}}, B_{j}^{f_{2}}\right) .
\end{aligned}
$$

Using Definition 6.9 again we have $\left(F_{21}\right)_{\square}(\sigma)=\sum_{j=0}^{m} P \times_{B_{i}^{f_{1}}} \overline{\mathcal{M}}_{F}\left(B_{i}^{f_{1}}, B_{j}^{f_{2}}\right)$, and hence by Definition 4.6, Definition 5.4 and Definition 6.5

$$
\begin{array}{r}
\partial_{0}\left(F_{21}\right)_{\square}(\sigma)=(-1)^{p+i} \sum_{j=0}^{m} \partial\left(P \times_{B_{i}^{f_{1}}} \overline{\mathcal{M}}_{F}\left(B_{i}^{f_{1}}, B_{j}^{f_{2}}\right)\right) \\
=(-1)^{p+i} \sum_{j=0}^{m}\left[\partial P \times_{B_{i}^{f_{1}}} \overline{\mathcal{M}}_{F}\left(B_{i}^{f_{1}}, B_{j}^{f_{2}}\right)\right. \\
\left.\quad+(-1)^{p+b_{i}^{f_{1}}} P \times_{B_{i}^{f_{1}}} \partial \overline{\mathcal{M}}_{F}\left(B_{i}^{f_{1}}, B_{j}^{f_{2}}\right)\right]
\end{array}
$$




$$
\begin{aligned}
=(-1)^{p+i} \sum_{j=0}^{m} \partial P \times_{B_{i}^{f_{1}}} \overline{\mathcal{M}}_{F}\left(B_{i}^{f_{1}}, B_{j}^{f_{2}}\right) \\
\quad+(-1)^{i+b_{i}^{f_{1}}} \sum_{j=0}^{m} P \times_{B_{i}^{f_{1}}} \partial \overline{\mathcal{M}}_{F}\left(B_{i}^{f_{1}}, B_{j}^{f_{2}}\right) \\
=(-1)^{p+i} \sum_{j=0}^{m} \partial P \times_{B_{i}^{f_{1}}} \overline{\mathcal{M}}_{F}\left(B_{i}^{f_{1}}, B_{j}^{f_{2}}\right) \\
\quad+\sum_{j=0}^{m} \sum_{k<i} P \times_{B_{i}^{f_{1}}} \overline{\mathcal{M}}_{f_{1}}\left(B_{i}^{f_{1}}, B_{k}^{f_{1}}\right) \times_{B_{k}^{f_{1}}} \overline{\mathcal{M}}_{F}\left(B_{k}^{f_{1}}, B_{j}^{f_{2}}\right) \\
\quad-\sum_{j=0}^{m} \sum_{j<q} P \times_{B_{i}^{f_{1}}} \overline{\mathcal{M}}_{F}\left(B_{i}^{f_{1}}, B_{q}^{f_{2}}\right) \times_{B_{q}^{f_{2}}} \overline{\mathcal{M}}_{f_{2}}\left(B_{q}^{f_{2}}, B_{j}^{f_{2}}\right) .
\end{aligned}
$$

Since

$$
\begin{aligned}
\left(\partial_{1} \oplus\right. & \left.\cdots \oplus \partial_{m}\right)\left(\left(F_{21}\right)_{\square}(\sigma)\right) \\
& =\sum_{0 \leq k<j \leq m} P \times_{B_{i}^{f_{1}}} \overline{\mathcal{M}}_{F}\left(B_{i}^{f_{1}}, B_{j}^{f_{2}}\right) \times_{B_{j}^{f_{2}}} \overline{\mathcal{M}}_{f_{2}}\left(B_{j}^{f_{2}}, B_{k}^{f_{2}}\right) \\
& =\sum_{j=0}^{m} \sum_{j<q} P \times_{B_{i}^{f_{1}}} \overline{\mathcal{M}}_{F}\left(B_{i}^{f_{1}}, B_{q}^{f_{2}}\right) \times_{B_{q}^{f_{2}}} \overline{\mathcal{M}}_{f_{2}}\left(B_{q}^{f_{2}}, B_{j}^{f_{2}}\right)
\end{aligned}
$$

we have $\left(F_{21}\right)_{\square}(\partial(\sigma))=\left(\partial_{0} \oplus \cdots \oplus \partial_{m}\right)\left(\left(F_{21}\right)_{\square}(\sigma)\right) \in \tilde{C}_{*}\left(f_{2}\right)$, and hence $\left(F_{21}\right)_{\square}(\partial(\sigma))$ $=\partial\left(F_{21}\right) \square(\sigma) \in C_{*}\left(f_{2}\right)$.

Corollary 6.12. The map $F_{21}: M \times \mathbb{R} \rightarrow \mathbb{R}$ induces a homomorphism in homology,

$$
\left(F_{21}\right)_{*}: H_{*}\left(C_{*}\left(f_{1}\right), \partial\right) \rightarrow H_{*}\left(C_{*}\left(f_{2}\right), \partial\right),
$$

which is independent of the choice of representing chain system.

6.4. Chain homotopies from time dependent gradient flows. Assume now that we have four Morse-Bott-Smale functions $f_{k}: M \rightarrow \mathbb{R}$, where $k=1,2,3,4$. By Proposition 6.11 these four functions determine chain maps

$$
\left(F_{l k}\right)_{\square}: C_{*}\left(f_{k}\right) \rightarrow C_{*}\left(f_{l}\right)
$$

for all $k, l=1,2,3,4$. We will show that the moduli spaces of gradient flow lines of a function $H: M \times \mathbb{R} \times \mathbb{R} \rightarrow \mathbb{R}$ meeting certain transversality requirements can be used to construct a chain homotopy between $\left(F_{43}\right) \square \circ\left(F_{31}\right) \square$ and $\left(F_{42}\right) \square \circ\left(F_{21}\right) \square$.

Following [4, 21], 40], and 44] we let $H: M \times \mathbb{R} \times \mathbb{R} \rightarrow \mathbb{R}$ be a smooth function that is strictly decreasing in its last two components such that for some large $T \gg 0$ we have

$$
H(x, s, t)=\left\{\begin{array}{lllll}
f_{1}(x)-\rho(s)-\rho(t) & \text { if } & s<-T & \text { and } & t<-T, \\
f_{2}(x)-\rho(s)-\rho(t) & \text { if } & s>T & \text { and } & t<-T, \\
f_{3}(x)-\rho(s)-\rho(t) & \text { if } & s<-T & \text { and } & t>T, \\
f_{4}(x)-\rho(s)-\rho(t) & \text { if } & s>T & \text { and } & t>T,
\end{array}\right.
$$

where $\rho: \mathbb{R} \rightarrow(-1,1)$ is a smooth strictly increasing function such that $\lim _{t \rightarrow-\infty} \rho(t)$ $=-1$ and $\lim _{t \rightarrow \infty} \rho(t)=1$. Taking the standard metric on $\mathbb{R}$ and the product met- 
ric on $M \times \mathbb{R} \times \mathbb{R}$, the gradient flow of $H: M \times \mathbb{R} \times \mathbb{R} \rightarrow \mathbb{R}$ on the last two components of its domain can be pictured in $\overline{\mathbb{R}} \times \overline{\mathbb{R}}$ as follows.

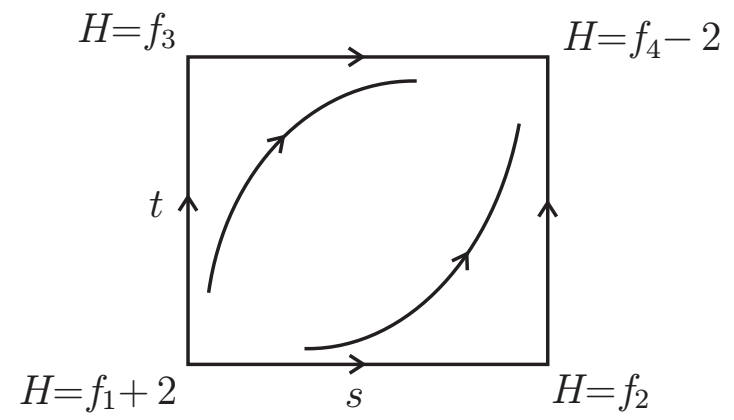

Let $\varphi_{\alpha}: M \times \mathbb{R} \times \mathbb{R} \rightarrow M \times \mathbb{R} \times \mathbb{R}$ denote the flow associated to $-\nabla H$ with respect to the product metric on $M \times \mathbb{R} \times \mathbb{R}$, and denote the stable and unstable manifolds of the critical point sets $B_{j}^{f_{4}}$ and $B_{i}^{f_{1}}$ by

$$
\begin{aligned}
W_{H}^{s}\left(B_{j}^{f_{4}}\right) & =\left\{(x, s, t) \in M \times \mathbb{R} \times \mathbb{R} \mid \lim _{\alpha \rightarrow+\infty} \pi_{1}\left(\varphi_{\alpha}(x, s, t)\right) \in B_{j}^{f_{4}}\right\}, \\
W_{H}^{u}\left(B_{i}^{f_{1}}\right) & =\left\{(x, s, t) \in M \times \mathbb{R} \times \mathbb{R} \mid \lim _{\alpha \rightarrow-\infty} \pi_{1}\left(\varphi_{\alpha}(x, s, t)\right) \in B_{i}^{f_{1}}\right\},
\end{aligned}
$$

where $\pi_{1}: M \times \mathbb{R} \times \mathbb{R} \rightarrow M$ denotes projection onto the first component. These stable and unstable manifolds inherit orientations from the stable and unstable manifolds of $f_{1}$ and $f_{4}$, and since both $f_{1}$ and $f_{4}$ satisfy the Morse-Bott-Smale transversality condition we can choose $H$ so that $W_{H}^{u}\left(B_{i}^{f_{1}}\right) \pitchfork W_{H}^{s}\left(B_{j}^{f_{4}}\right)$ is an oriented submanifold of $M \times \mathbb{R} \times \mathbb{R}$ for all $i, j=0, \ldots, m$.

The moduli spaces of gradient flow lines of $H$ are defined to be

$$
\mathcal{M}_{H}\left(B_{i}^{f_{1}}, B_{j}^{f_{4}}\right)=\left(W_{H}^{u}\left(B_{i}^{f_{1}}\right) \cap W_{F}^{s}\left(B_{j}^{f_{4}}\right)\right) / \mathbb{R}
$$

for all $i, j=0, \ldots, m$, where the $\mathbb{R}$-action is defined by the negative gradient flow, and we have

$$
\operatorname{dim} \mathcal{M}_{H}\left(B_{i}^{f_{1}}, B_{j}^{f_{4}}\right)=b_{i}^{f_{1}}+i-j+1 .
$$

Moreover, there are gluing and compactification results for the moduli spaces of $H$ similar to those stated above. Hence, the moduli space $\mathcal{M}_{H}\left(B_{i}^{f_{1}}, B_{j}^{f_{4}}\right)$ has a compactification $\overline{\mathcal{M}}_{H}\left(B_{i}^{f_{1}}, B_{j}^{f_{4}}\right)$, consisting of all the piecewise gradient flow lines of $H$ from $B_{i}^{f_{1}}$ to $B_{j}^{f_{4}}$, which is a compact smooth manifold with corners. The beginning and endpoint maps $\partial_{-}$and $\partial_{+}$extend to smooth maps on the compactified space.

Theorem 6.13. The function $H: M \times \mathbb{R} \times \mathbb{R} \rightarrow \mathbb{R}$ induces a chain homotopy

$$
\left(F_{43}\right)_{\square} \circ\left(F_{31}\right)_{\square}-\left(F_{42}\right)_{\square} \circ\left(F_{21}\right)_{\square}=\partial H_{\square}+H_{\square} \partial .
$$

Proof. Assume that the components of $B_{i}^{f_{1}}$ are of the same dimension and that appropriate representing chains have been chosen. The boundary of $\overline{\mathcal{M}}_{H}\left(B_{i}^{f_{1}}, B_{j}^{f_{4}}\right)$ is $(-1)^{i+b_{i}^{f_{1}}}$ times the quantity

$$
\sum_{k=0}^{m} \overline{\mathcal{M}}_{F_{21}}\left(B_{i}^{f_{1}}, B_{k}^{f_{2}}\right) \times_{B_{k}^{f_{2}}} \overline{\mathcal{M}}_{F_{42}}\left(B_{k}^{f_{2}}, B_{j}^{f_{4}}\right)
$$




$$
\begin{aligned}
& -\sum_{n=0}^{m} \overline{\mathcal{M}}_{F_{31}}\left(B_{i}^{f_{1}}, B_{n}^{f_{3}}\right) \times_{B_{n}^{f_{3}}} \overline{\mathcal{M}}_{F_{43}}\left(B_{n}^{f_{3}}, B_{j}^{f_{4}}\right) \\
& +\sum_{n<i} \overline{\mathcal{M}}_{f_{1}}\left(B_{i}^{f_{1}}, B_{n}^{f_{1}}\right) \times_{B_{n}^{f_{1}}} \overline{\mathcal{M}}_{H}\left(B_{n}^{f_{1}}, B_{j}^{f_{4}}\right) \\
& +\sum_{j<n} \overline{\mathcal{M}}_{H}\left(B_{i}^{f_{1}}, B_{n}^{f_{4}}\right) \times_{B_{n}^{f_{4}}} \overline{\mathcal{M}}_{f_{4}}\left(B_{n}^{f_{4}}, B_{j}^{f_{4}}\right) .
\end{aligned}
$$

If $\sigma: P \rightarrow B_{i}^{f_{1}}$ satisfies the required transversality requirements, then $H_{\square}(\sigma) \in$ $C_{*}\left(f_{4}\right)$ is represented by the abstract topological chain

$$
\sum_{j=0}^{m} P \times_{B_{i}^{f_{1}}} \overline{\mathcal{M}}_{H}\left(B_{i}^{f_{1}}, B_{j}^{f_{4}}\right)
$$

and $\partial H_{\square}(\sigma) \in C_{*}\left(f_{4}\right)$ is represented by the abstract topological chain

$$
\begin{aligned}
& \sum_{j=0}^{m}\left(\partial_{0}\left(P \times_{B_{i}^{f_{1}}} \overline{\mathcal{M}}_{H}\left(B_{i}^{f_{1}}, B_{j}^{f_{4}}\right)\right)\right. \\
& \left.\quad+\sum_{k<j} P \times_{B_{i}^{f_{1}}} \overline{\mathcal{M}}_{H}\left(B_{i}^{f_{1}}, B_{j}^{f_{4}}\right) \times_{B_{j}^{f_{4}}} \overline{\mathcal{M}}_{f_{4}}\left(B_{j}^{f_{4}}, B_{k}^{f_{4}}\right)\right) .
\end{aligned}
$$

For the $\partial_{0}$ terms in the above sum we have

$$
\begin{aligned}
\partial_{0} & \left(P \times_{B_{i}^{f_{1}}} \overline{\mathcal{M}}_{H}\left(B_{i}^{f_{1}}, B_{j}^{f_{4}}\right)\right) \\
& =(-1)^{p+i+1}\left(\partial P \times_{B_{i}^{f_{1}}} \overline{\mathcal{M}}_{H}\left(B_{i}^{f_{1}}, B_{j}^{f_{4}}\right)+(-1)^{p+b_{i}^{f_{1}}} P \times_{B_{i}^{f_{1}}} \partial \overline{\mathcal{M}}_{H}\left(B_{i}^{f_{1}}, B_{j}^{f_{4}}\right)\right),
\end{aligned}
$$

where $\partial \overline{\mathcal{M}}_{H}\left(B_{i}^{f_{1}}, B_{j}^{f_{4}}\right)$ is the sum given above. In addition, the term $H_{\square}(\partial \sigma)$ is represented by the abstract topological chain

$$
\begin{aligned}
& \sum_{j=0}^{m}\left((-1)^{p+i} \partial P \times_{B_{i}^{f_{1}}} \overline{\mathcal{M}}_{H}\left(B_{i}^{f_{1}}, B_{j}^{f_{4}}\right)\right. \\
& \left.\quad+\sum_{n<i} P \times_{B_{i}^{f_{1}}} \overline{\mathcal{M}}_{f_{1}}\left(B_{i}^{f_{1}}, B_{n}^{f_{1}}\right) \times_{B_{n}^{f_{1}}} \overline{\mathcal{M}}_{H}\left(B_{n}^{f_{1}}, B_{j}^{f_{4}}\right)\right) .
\end{aligned}
$$

Hence, $\partial H_{\square}(\sigma)+H_{\square}(\partial \sigma)$ is represented by the sum from $j=0$ to $m$ of the abstract topological chains

$$
\begin{aligned}
& \sum_{n=0}^{m} P \times_{B_{i}^{f_{1}}} \overline{\mathcal{M}}_{F_{31}}\left(B_{i}^{f_{1}}, B_{n}^{f_{3}}\right) \times_{B_{n}^{f_{3}}} \overline{\mathcal{M}}_{F_{43}}\left(B_{n}^{f_{3}}, B_{j}^{f_{4}}\right) \\
& \quad-\sum_{k=0}^{m} P \times_{B_{i}^{f_{1}}} \overline{\mathcal{M}}_{F_{21}}\left(B_{i}^{f_{1}}, B_{k}^{f_{2}}\right) \times_{B_{k}^{f_{2}}} \overline{\mathcal{M}}_{F_{42}}\left(B_{k}^{f_{2}}, B_{j}^{f_{4}}\right) .
\end{aligned}
$$

Corollary 6.14. For every Morse-Bott-Smale function $f: M \rightarrow \mathbb{R}$ there exists a smooth function $F: M \times \mathbb{R} \rightarrow \mathbb{R}$ such that

$$
F_{*}: H_{*}\left(C_{*}(f), \partial\right) \rightarrow H_{*}\left(C_{*}(f), \partial\right)
$$

is the identity homomorphism. 
Proof. Let $F: M \times \mathbb{R} \rightarrow \mathbb{R}$ be defined as $F(x, t)=f(x)-\rho(t)$ for all $(x, t) \in M \times \mathbb{R}$. If $\sigma: P \rightarrow B_{i}^{f}$ is a singular $C_{p}^{f}$-space in $B_{i}^{f}$, then

$$
F_{\square}(\sigma)=\sum_{j \leq i}\left(\partial_{+}: P \times_{B_{i}^{f}} \overline{\mathcal{M}}_{F}\left(B_{i}^{f}, B_{j}^{f}\right) \rightarrow B_{j}^{f}\right),
$$

since the Morse-Bott index of a Morse-Bott-Smale function is decreasing along its gradient flow lines by Lemma 3.6. The moduli space $\overline{\mathcal{M}}_{F}\left(B_{i}^{f}, B_{i}^{f}\right)$ is diffeomorphic to $B_{i}^{f}$, and the beginning point map $\partial_{-}: \overline{\mathcal{M}}_{F}\left(B_{i}^{f}, B_{i}^{f}\right) \rightarrow B_{i}^{f}$ is the identity map with respect to this diffeomorphism. Hence,

$$
P \times_{B_{i}^{f}} \overline{\mathcal{M}}_{F}\left(B_{i}^{f}, B_{i}^{f}\right) \approx\left\{(x, y) \in P \times B_{i}^{f} \mid \sigma(x)=y\right\} \approx P,
$$

and the endpoint map $\partial_{+}: P \times_{B_{i}^{f}} \overline{\mathcal{M}}_{F}\left(B_{i}^{f}, B_{i}^{f}\right) \rightarrow B_{i}^{f}$ agrees with $\sigma: P \rightarrow B_{i}^{f}$. Therefore,

$$
F_{\square}(\sigma)=\sigma+\sum_{j<i}\left(\partial_{+}: P \times_{B_{i}^{f}} \overline{\mathcal{M}}_{F}\left(B_{i}^{f}, B_{j}^{f}\right) \rightarrow B_{j}^{f}\right) .
$$

Note that if $f$ is a Morse-Smale function or a constant function, then the terms in the above sum with $j<i$ are all zero in $C_{*}(f)$, and thus the map $F_{\square}: C_{*}(f) \rightarrow C_{*}(f)$ is the identity at the level of chains.

In general, let $\tilde{F}: M \times \mathbb{R} \rightarrow \mathbb{R}$ be a smooth function that is strictly decreasing in its second component such that for some large $T \gg 0$ we have

$$
\tilde{F}(x, t)=\left\{\begin{array}{llc}
f(x)-\rho(t) & \text { if } & t<-\frac{1}{2} T \\
h_{t}(x) & \text { if } & -\frac{1}{2} T \leq t \leq \frac{1}{2} T, \\
f(x)-\rho(t) & \text { if } & t>\frac{1}{2} T
\end{array}\right.
$$

where the approximation $h_{t}(x)$ is chosen so that the non-constant time dependent gradient flow lines of $\tilde{F}$ all flow into $W^{s}\left(B_{0}^{f}\right) \times \mathbb{R}$. This is possible because $\operatorname{dim} W^{s}\left(B_{0}^{f}\right)=m$, and every piecewise gradient flow line of $f$ can be extended to a piecewise gradient flow line that ends in $B_{0}^{f}$. If $\sigma: P \rightarrow B_{i}^{f}$ is a singular $C_{p}^{f}$-space in $B_{i}^{f}$, then

$$
\tilde{F}_{\square}(\sigma)=\sigma+\left(\partial_{+}: P \times_{B_{i}^{f}} \overline{\mathcal{M}}_{\tilde{F}}\left(B_{i}^{f}, B_{0}^{f}\right) \rightarrow B_{0}^{f}\right) .
$$

Now, let $f_{1}=f_{2}=f_{4}=f$, and let $f_{3} \equiv 0$ be a constant function. Choose

$$
F_{31}(x, t)=\left\{\begin{array}{llc}
f(x)-\rho(t) & \text { if } & t<-T, \\
\mu(t) f(x)-\rho(t) & \text { if } & -T \leq t \leq-\frac{1}{2} T, \\
0-\rho(t) & \text { if } & t>-\frac{1}{2} T,
\end{array}\right.
$$

where $\mu: \mathbb{R} \rightarrow[0,1]$ is a smooth decreasing function that is 1 for $t \leq-T$ and 0 for $t \geq-\frac{1}{2} T$. If we choose

$$
F_{43}(x, t)=\left\{\begin{array}{llc}
0-\rho(t) & \text { if } & t<-T, \\
(1-\mu(t)) f(x)-\rho(t) & \text { if } & -T \leq t \leq-\frac{1}{2} T, \\
h_{t}(x) & \text { if } & -\frac{1}{2} T \leq t \leq \frac{1}{2} T, \\
f(x)-\rho(t) & \text { if } & t>\frac{1}{2} T,
\end{array}\right.
$$

then for all $i=0, \ldots, m$ we have diffeomorphisms

$$
\overline{\mathcal{M}}_{F_{31}}\left(B_{i}^{f}, M\right) \times_{M} \overline{\mathcal{M}}_{F_{43}}\left(M, B_{0}^{f}\right) \approx \overline{\mathcal{M}}_{\tilde{F}}\left(B_{i}^{f}, B_{0}^{f}\right),
$$

and hence, $\left(F_{43}\right)_{\square} \circ\left(F_{31}\right)_{\square}=\tilde{F}_{\square}$. Thus, if we choose $F_{21}=F_{42}=\tilde{F}$, then Theorem 6.13 implies that $\tilde{F}_{*}=\tilde{F}_{*} \circ \tilde{F}_{*}$. 
If $\sum_{k} n_{k} \sigma_{k}$ is a cycle in $C_{*}(f)$, then we have

$$
\tilde{F}_{*}\left(\left[\sum_{k} n_{k} \sigma_{k}\right]\right)=\left[\sum_{k} n_{k} \sigma_{k}\right]+[C],
$$

where $C$ is a sum of singular spaces in $B_{0}^{f}$ of the form $\partial_{+}: P_{k} \times_{B_{i}^{f}} \overline{\mathcal{M}}_{\tilde{F}}\left(B_{i_{k}}^{f}, B_{0}^{f}\right) \rightarrow$ $B_{0}^{f}$. Hence,

$$
\left(\tilde{F}_{*} \circ \tilde{F}_{*}\right)\left(\left[\sum_{k} n_{k} \sigma_{k}\right]\right)=\left[\sum_{k} n_{k} \sigma_{k}\right]+2[C],
$$

and the fact that $\tilde{F}_{*}=\tilde{F}_{*} \circ \tilde{F}_{*}$ implies that $[C]=0$. Therefore,

$$
\tilde{F}_{*}\left(\left[\sum_{k} n_{k} \sigma_{k}\right]\right)=\left[\sum_{k} n_{k} \sigma_{k}\right],
$$

and $\tilde{F}_{*}: H_{*}\left(C_{*}(f), \partial\right) \rightarrow H_{*}\left(C_{*}(f), \partial\right)$ is the identity homomorphism.

Corollary 6.15. For any two Morse-Bott-Smale functions $f_{1}, f_{2}: M \rightarrow \mathbb{R}$ the time dependent gradient flow lines from $f_{1}$ to $f_{2}$ determine a canonical homomorphism

$$
\left(F_{21}\right)_{*}: H_{*}\left(C_{*}\left(f_{1}\right), \partial\right) \rightarrow H_{*}\left(C_{*}\left(f_{2}\right), \partial\right),
$$

i.e. the map $\left(F_{21}\right)_{*}$ is independent of the choice of the function $F_{21}: M \times \mathbb{R} \rightarrow \mathbb{R}$ and the representing chain system used to define the chain map $\left(F_{21}\right) \square: C_{*}\left(f_{1}\right) \rightarrow$ $C_{*}\left(f_{2}\right)$.

Proof. Let $f_{2}=f_{3}=f_{4}$ in Theorem 6.13, let $F_{21}: M \times \mathbb{R} \rightarrow \mathbb{R}$ and $F_{31}: M \times \mathbb{R} \rightarrow \mathbb{R}$ be two functions that define time-dependent gradient flow lines from $f_{1}$ to $f_{2}=f_{3}$, and let $F_{22}$ and $F_{33}$ be the functions from Corollary 6.14. Theorem 6.13 implies

$$
\left(F_{33}\right)_{*} \circ\left(F_{31}\right)_{*}=\left(F_{22}\right)_{*} \circ\left(F_{21}\right)_{*},
$$

and since $\left(F_{22}\right)_{*}=\left(F_{33}\right)_{*}=i d$, we have $\left(F_{31}\right)_{*}=\left(F_{21}\right)_{*}$.

Corollary 6.16. For any four Morse-Bott-Smale functions $f_{k}: M \rightarrow \mathbb{R}$, where $k=1,2,3,4$, the canonical homomorphisms satisfy

$$
\left(F_{43}\right)_{*} \circ\left(F_{31}\right)_{*}=\left(F_{42}\right)_{*} \circ\left(F_{21}\right)_{*}
$$

and

$$
\left(F_{32}\right)_{*} \circ\left(F_{21}\right)_{*}=\left(F_{31}\right)_{*} .
$$

Proof. This follows immediately from Theorem 6.13 and Corollary 6.14.

Theorem 6.17. The homology of the Morse-Bott chain complex $\left(C_{*}(f), \partial\right)$ is independent of the Morse-Bott-Smale function $f: M \rightarrow \mathbb{R}$. Therefore,

$$
H_{*}\left(C_{*}(f), \partial\right) \approx H_{*}(M ; \mathbb{Z}) .
$$

Proof. Let $f_{1}, f_{2}: M \rightarrow \mathbb{R}$ be Morse-Bott-Smale functions. If we take $f_{3}=f_{1}$ in Corollary 6.16, then we see that $\left(F_{12}\right)_{*} \circ\left(F_{21}\right)_{*}=\left(F_{11}\right)_{*}=i d$ by Corollaries 6.14 and 6.15. Similarly, $\left(F_{21}\right)_{*} \circ\left(F_{12}\right)_{*}=i d$. 


\section{REFERENCES}

1. M. F. Atiyah, Convexity and commuting Hamiltonians, Bull. London Math. Soc. 14 (1982), no. 1, 1-15. MR83e:53037

2. M. F. Atiyah and R. Bott, The Yang-Mills equations over Riemann surfaces, Philos. Trans. Roy. Soc. London Ser. A 308 (1983), no. 1505, 523-615. MR85k:14006

3. Michèle Audin, Torus actions on symplectic manifolds, revised ed., Progress in Mathematics, vol. 93, Birkhäuser Verlag, Basel, 2004. MR2091310 (2005k:53158)

4. David M. Austin and Peter J. Braam, Morse-Bott theory and equivariant cohomology, The Floer memorial volume, Progr. Math., vol. 133, Birkhäuser, Basel, 1995, pp. 123-183. MR.1362827 (96i:57037)

5. _ Equivariant Floer theory and gluing Donaldson polynomials, Topology 35 (1996), no. 1, 167-200. MR.1367280 (97e:57037)

6. Augustin Banyaga and David E. Hurtubise, The Morse-Bott inequalities via a dynamical systems approach, Ergodic Theory Dynam. Systems 29 (2009), no. 6, 1693-1703. MR.2563088

7. L Lectures on Morse homology, Kluwer Texts in the Mathematical Sciences, vol. 29, Kluwer Academic Publishers Group, Dordrecht, 2004. MR2145196 (2006i:58016)

8. , A proof of the Morse-Bott lemma, Expo. Math. 22 (2004), no. 4, 365-373. MR2075744 (2005b:57062)

9. Jean-François Barraud and Octav Cornea, Lagrangian intersections and the Serre spectral sequence, Ann. of Math. (2) 166 (2007), no. 3, 657-722. MR2373371 (2008j:53149)

10. Stefan Bauer, Electronic posting, Topology Listserv (2005).

11. Raoul Bott, Non-Degenerate critical manifolds, Ann. of Math. (2) 60 (1954), 248-261. MR0064399 (16:276f)

12. Raoul Bott and Hans Samelson, Applications of the theory of Morse to symmetric spaces, Amer. J. Math. 80 (1958), 964-1029. MR0105694 (21:4430)

13. F. Bourgeois, Y. Eliashberg, H. Hofer, K. Wysocki, and E. Zehnder, Compactness results in symplectic field theory, Geom. Topol. 7 (2003), 799-888 (electronic). MR.2026549 (2004m:53152)

14. Glen E. Bredon, Topology and geometry, Graduate Texts in Mathematics, vol. 139, SpringerVerlag, New York, 1993. MR1224675 (94d:55001)

15. J. L. Bryant, Triangulation and general position of PL diagrams, Topology Appl. 34 (1990), no. 3, 211-233. MR1042280 (91b:57032)

16. S. K. Donaldson, Floer homology groups in Yang-Mills theory, Cambridge Tracts in Mathematics, vol. 147, Cambridge University Press, Cambridge, 2002. With the assistance of M. Furuta and D. Kotschick. MR.1883043 (2002k:57078)

17. Michael Farber, Topology of closed one-forms, Mathematical Surveys and Monographs, vol. 108, American Mathematical Society, Providence, RI, 2004. MR2034601 (2005c:58023)

18. A. Floer and H. Hofer, Coherent orientations for periodic orbit problems in symplectic geometry, Math. Z. 212 (1993), no. 1, 13-38. MR.1200162 (94m:58036)

19. Andreas Floer, An instanton-invariant for 3-manifolds, Comm. Math. Phys. 118 (1988), no. 2, 215-240. MR.956166 (89k:57028)

20. __ Morse theory for Lagrangian intersections, J. Differential Geom. 28 (1988), no. 3, 513-547. MR90f:58058

21. Symplectic fixed points and holomorphic spheres, Comm. Math. Phys. 120 (1989), no. 4, 575-611. MR90e:58047

22. Urs Frauenfelder, The Arnold-Givental conjecture and moment Floer homology, Int. Math. Res. Not. (2004), no. 42, 2179-2269. MR2076142 (2005g:53174)

23. Kenji Fukaya, Floer homology of connected sum of homology 3-spheres, Topology 35 (1996), no. 1, 89-136. MR.1367277(97d:57041)

24. Mikhael Gromov, Filling Riemannian manifolds, J. Differential Geom. 18 (1983), no. 1, 1147. MR697984 (85h:53029)

25. F. Reese Harvey and H. Blaine Lawson, Jr., Finite volume flows and Morse theory, Ann. of Math. (2) 153 (2001), no. 1, 1-25. MR.1826410 (2002c:58018)

26. H. Hofer, K. Wysocki, and E. Zehnder, A general Fredholm theory II: Implicit function theorems, Geom. Funct. Anal. To appear.

27. _ A general Fredholm theory I: A splicing-based differential geometry, J. Eur. Math. Soc. (JEMS) 9 (2007), no. 4, 841-876. MR2341834 (2008m:53202) 
28. David E. Hurtubise, The flow category of the action functional on $\mathcal{L} G_{n, n+k}(\mathbb{C})$, Illinois J. Math. 44 (2000), no. 1, 33-50. MR1731380 (2001i:57047)

29. _ Multicomplexes and spectral sequences, J. Algebra Appl. To appear.

30. Peter J. Kahn, Pseudohomology and homology, arXiv:math/0111223v1 (2001).

31. Frances Clare Kirwan, Cohomology of quotients in symplectic and algebraic geometry, Mathematical Notes, vol. 31, Princeton University Press, Princeton, NJ, 1984. MR86i:58050

32. Janko Latschev, Gradient flows of Morse-Bott functions, Math. Ann. 318 (2000), no. 4, 731759. MR1802508 (2001m:58026)

33. Gang Liu and Gang Tian, On the equivalence of multiplicative structures in Floer homology and quantum homology, Acta Math. Sin. (Engl. Ser.) 15 (1999), no. 1, 53-80. MR.1701133 (2001g:53154)

34. Juan Margalef Roig and Enrique Outerelo Domínguez, Differential topology, North-Holland Mathematics Studies, vol. 173, North-Holland Publishing Co., Amsterdam, 1992. MR1173211 (93g:58005)

35. William S. Massey, A basic course in algebraic topology, Graduate Texts in Mathematics, vol. 127, Springer-Verlag, New York, 1991. MR.1095046 (92c:55001)

36. Dusa McDuff and Dietmar Salamon, J-holomorphic curves and symplectic topology, American Mathematical Society Colloquium Publications, vol. 52, American Mathematical Society, Providence, RI, 2004. MR2045629 (2004m:53154)

37. Jean-Pierre Meyer, Acyclic models for multicomplexes, Duke Math. J. 45 (1978), no. 1, 67-85. MR.486489(80b:55012)

38. Lars Tyge Nielsen, Transversality and the inverse image of a submanifold with corners, Math. Scand. 49 (1981), no. 2, 211-221 (1982). MR661891 (83m:57022)

39. Yongbin Ruan and Gang Tian, Bott-type symplectic Floer cohomology and its multiplication structures, Math. Res. Lett. 2 (1995), no. 2, 203-219. MR.1324703(96a:57074)

40. Dietmar Salamon, Lectures on Floer homology, Symplectic geometry and topology (Park City, UT, 1997), IAS/Park City Math. Ser., vol. 7, Amer. Math. Soc., Providence, RI, 1999, pp. 143-229. MR2000g:53100

41. Matthias Schwarz, Morse homology, Birkhäuser Verlag, Basel, 1993. MR.1239174 (95a:58022)

42. _ Equivalences for Morse homology, Geometry and topology in dynamics (WinstonSalem, NC, 1998/San Antonio, TX, 1999), Contemp. Math., vol. 246, Amer. Math. Soc., Providence, RI, 1999, pp. 197-216. MR1732382 (2000j:57070)

43. Frank W. Warner, Foundations of differentiable manifolds and Lie groups, Graduate Texts in Mathematics, vol. 94, Springer-Verlag, New York, 1983. MR722297 (84k:58001)

44. Joa Weber, The Morse-Witten complex via dynamical systems, Expo. Math. 24 (2006), no. 2, 127-159. MR2243274

Department of Mathematics, The Pennsylvania State University, University Park, University Park, Pennsylvania 16802

E-mail address: banyaga@math.psu.edu

Department of Mathematics and Statistics, The Pennsylvania State University, Altoona, Altoona, Pennsylvania 16601-3760

E-mail address: Hurtubise@psu.edu 